\title{
TENSIONS AND CHALLENGES
}

\section{In Architectural Practice}

\author{
BY \\ CLAIRE WARNOCK
}

\begin{abstract}
A thesis
submitted to the Victoria University of Wellington in fulfilment of the requirements for the degree of Master of Architecture
\end{abstract}

Victoria University of Wellington 2019 



\section{i Abstract}

This thesis builds on existing research which examines the impact of architectural culture and the changing role of the profession, recognising that there is a need for drastic change in the way in which the architect's role is comprehended. The thesis follows a qualitative research methodology, considering key issues emerging from theoretical research, advancing understanding of these issues through in-depth semistructured interviews with architects working in both New Zealand and the United Kingdom. The thesis begins with a review of current literature to situate the study within a theoretical framework and to direct the content of the qualitative research. What is made evident through this section, is the increasing gap between the image of the architect and the actuality of architectural practice. Next, the thesis reports on the qualitative research undertaken, the data collected and the results of the analysis. Architects were asked to describe their experiences of the tensions and challenges affecting their role and to comment on how they negotiate these through how they work. The results of the research shows that the architects interviewed are less invested in the image of the architect than the creative processes involved in delivering a project, and that a focus on image obscures the significance of this process. The study concludes that the creative processes inherent in the architect's vocation support innovative and adaptive working; thus, architects are equipped with the skills to evolve their role from within, to become facilitators to the requirements of the changing context. 



\section{ii Table of Contents}
i.
Abstract
ii. Table of Contents
iii. Preface
iv. Acknowledgements

Chapters

1. Introduction and Methodology

1.1 Context for the Study 1

1.2 Motivation for the Study 2

1.3 Key Themes 4

1.4 Aim and Scope of the Thesis 5

1.5 Research Approach 7

1.6 Overview of the Thesis 17

2. Constructing the Image of the Architect

2.1 Introduction 19

2.2 Defining an Architect 20

2.3 The Renaissance Artist 22

2.4 Enlightenment and Autonomy 24

2.5 The Industrial Revolution 28

2.6 The Modern Movement 32

2.7 Concluding Discussion 36

3. Literature Review: Tension and Negotiation

$\begin{array}{llc}3.1 & \text { Introduction } & 37 \\ 3.2 & \text { Tension } & 37 \\ 3.3 & \text { Challenge } & 41 \\ 3.4 & \text { Negotiation } & 46 \\ 3.5 & \text { Concluding Discussion } & 54\end{array}$ 
4. Research

4.1 Introduction $\quad 55$

4.2 The Experience of Image 56

4.2.1 Experiences of disparity in the image 59

4.2.2 Causes of disparity in the image 62

4.3 Tensions and Challenges 65

4.3.1 Gap between the image and actuality 65

4.3.2 Changing Context 68

4.3.3 Affects on the role 69

4.3.4 Review of tensions and challenges $\quad 74$

4.4 Negotiation 75

4.4.1 Challenging the image 75

4.4.2 Strategic thinking 78

4.4.3 Diversifying and specialising 81

4.4.4 Research and Critical Practice 83

4.4.5 Review of negotiation 86

4.5 Concluding discussions $\quad 86$

\section{Discussion and Conclusion}

5.1 Image 89

5.2 Context 91

5.3 Negotiation 92

5.4 Conclusion 94

v. References

vi. Appendix-Architects Selection Matrix

vii. Appendix-Sample Interview Questions 


\section{iii Preface}

"The architectural profession has professed itself to be the marriage of telos and techne, of concept and structure, of art and science. To divorce one part of the dialectic will most certainly trigger or reflect a fundamental change in the profession" (Cuff, 1992, p.212).

This thesis arose out of an issue that has troubled me for many years: what is the purpose of being an architect when the role is frequently undermined, motives are often questioned and when considered design work is relentlessly subjected to transient and anodyne causes? As a practicing architect in the UK, it became clear that the production of buildings, and therefore the emergence of the wider built environment, is a shared act with opaque lines of responsibility; in rather too many cases the result of a battle of wills and tough negotiation, rather than a coming together of compatible skills towards a common goal. Creative impotence can lead architects to be dissatisfied with the process and, often, the outcome. Overlapping roles and unclear boundaries create tensions within a project team and incompatible goals within the delivery hierarchy can lead to conflict. Before entering the career, and even as a student, these tensions and challenges were not clear to me. Thus, I entered the profession with an incomplete understanding of what the actuality of practice entails. 



\section{iv Acknowledgements}

The support and assistance of others has been essential, first to my embarking on this research project and then in persisting through this challenging yet rewarding year of study. My first thanks go to my supervisor Dr. Jan Smitheram for her unwavering support and patience. Jan's generosity with her time and judicious advice have been invaluable throughout this entire process. Thanks to Jan and her clear guidance, I have learned an enormous amount, for which I will be forever grateful.

I am also extremely grateful to the architects who agreed to participate in this research. Their engagement and enthusiasm to share their experiences led to a rich crop of information from which to draw the observations contained in this thesis.

Thank you to Graduate Women Wellington for supporting this study through their Masters by Thesis scholarship, which enabled me to put in place the support needed to help me to focus on writing this thesis in the crucial closing weeks.

Lastly to my family; to my Sister Ceri who has been the most amazing intellectual and emotional support and my Mother Barbara who is selfless, stalwart and an inspiration; without you both, this adventure would not have been possible. Thanks to Adam for Sundays; Arthur and Eloise who provided a loving distraction all the other days and of course to Reg, who as my walking buddy and constant companion at all hours of the day and night has inadvertently been party to the 'eureka' moments.

Without the support, input and advice from all of you, this research would have been an impenetrable, solitary and far less rewarding endeavor. Thank you. 



\section{CHAPTER 1}

\section{Introduction and Methodology}

The future role of the architect is changing and this is an issue which is attracting increasing analysis amidst both academic and professional bodies. This thesis explores both realms; first a theoretical study examines how the image of the architect evolved historically as a layering of identities, then a qualitative study examines the effects of changing context on architects in practice to understand how the architect's image has become disconnected from the actuality of architectural practice. This thesis, therefore, examines how the architect's role has changed and how it might continue to change in the future.

\subsection{CONTEXT FOR THE STUDY}

Studies into the image of the architect mainly grow from recent times, when the role of the architect came under scrutiny. Seminal research was undertaken on the image from a sociological perspective, commencing with Larson (1977), who identifies the special influence and prestige that architects acquired when the profession emerged. The image of the architect is subsequently discussed by both Blau (1984) and Gutman (1988) who explore challenges to the traditional role, arising from changes in the construction industry. These studies are contemporary to the shift in trend from government building programmes, to large scale market-led projects (Duffy \& Hutton, 1998), indicating the importance of context to the architect's evolving status. Symes, Eley \& Seidel (1995) contextualise this period as occurring from the late 1960s with the rise of:

...competition from other professions. A principal focus has been the growth of project management as a separate discipline. This worry has been linked with concerns about new technology, about the public view of architects (Symes et al., 1995, p.15).

Thus, changing context and scrutiny of the architect's image is considered material to changes in the architect's position (Cohen, Wilkinson, Arnold, \& Finn, 2005). 
More recent research into the architect's role covers a range of interrelated foci. Examinations of architectural culture (Stevens, 1998; Till, 2009) identify how conventions within the profession resist adaptation. These studies recognise that tension instigated by changing context is magnified by a profession that has not sufficiently adapted its image in response. Areas of research which focus on reconciling the tension between image and context explore closer links between practice and pedagogy (Cuff, 1991; Crysler, 1995; Harriss \& Froud, 2015; Samuel, 2018) whereas research with a practice focus, examines how the role might be redefined or even restructured to meet the challenges from changing context (Cuff, 1992; Tombesi, 2012; Hyde, 2012; Ahuja, Nikolova \& Clegg, 2017). Within the outlined framework of research, this thesis is located between a cultural examination of the image of the architect and qualitative explorations of architects' experiences in practice. The study, therefore, seeks to further understand the actuality of the architect's role and how it might develop in the future.

\subsection{MOTIVATION FOR THE STUDY}

This thesis proceeds under the supposition that the architect's role is valuable, whilst recognising its status is changing. Architecture can exist in isolation of architects, therefore, what can we learn from a study of the future role of the profession? Samuel (2018) observes the increasing profile of designers, such as Heatherwick and Assemble, who are notable players in contemporary architecture, but are not members of the architectural profession. Similarly, Awan, Schneider and Till (2013) acknowledge that it is not only architects that contribute creatively to the built environment. Consequently, protection of the architect's role through its professional status is no longer assured given the porosity of the architect's remit and particularly in a context where the assumed authority of experts is being interrogated (Carrio, 2015; Nichols, 2017; Clarke \& Newman, 2017; Larson, 2018).

Considering these arguments, the relationship between architecture and the architectural profession is more fluid than the image of the architect communicates. Burr and Jones (2010) identify that the public understanding of the architect's central purpose is to generate a building's design, yet if this is the sole function of an architect and architecture does not require architects, the role becomes superfluous. To resolve this quandary, this thesis is framed by an understanding that the role of the architect goes beyond the material production of a building; that the role has a cultural and ethical 
dimension and therefore a social value. Larson (2018), reflecting on her early work, observes:

professions were special: because they fulfilled important social needs, because they were communities, because they knew more than those who used their services, because they were trusted, because they pursued the clients' interest before their own (Larson, 2018, p.28).

The position that architects satisfy a distinct function is consistent with contemporary arguments, which maintain that architectural knowledge fulfils an ethical and moral dimension (Collier, 2006; Franck \& Lepori, 2007; Burr \& Jones, 2010) and, thus, by "building with thought, with philosophical, ethical and critical awareness" (Samuel, 2018, p.105) creates a social value. Recent opinion holds however, that if the role is to remain significant, architects must refocus; that the skills and knowledge offered by architects is not clearly communicated (Samuel, 2018), partly due to inappropriate preoccupations (Awan et al., 2013) and even that the protected title could be considered an impediment (Deamer, 2012, Reinmuth, 2017). The details of these propositions are unpacked during this study to explicate the elements which have enduring value in the architect's role.

A comprehensive argument in favour of the value of architects cannot reasonably be undertaken within the scope of this thesis, and in any event this argument is covered at length by Samuel in her recent publication Why Architects Matter (2018). However, acknowledging that the role is valuable whilst accepting it is also subject to change not only underlines the extent of tension surrounding the image of the architect, but also identifies which of the architect's skills have an enduring value. This study, therefore, explores how the role of the architect is changing by scrutinising the obstacles and then the opportunities for the architect's future direction and using three themes; image, context and negotiation, to do so.

\subsection{KEY THEMES}

Section 1.2 introduced the three key themes, image, context and negotiation, which recur throughout this thesis and are the focus of the discussion. This section discusses the themes, defining each concept within the setting of the study. 
The first premise of this thesis is that the image of the architect has evolved historically as a layering of identities, but within the contemporary context, the prevailing image no longer reflects the actuality of the role:

Do architects know themselves? If not, they cannot expect to predict or plan their own futures. Yet throughout the profession, people purvey and sustain a misleading impression of what it is to be an architect, buttressed in large measure by illusions of what it has been to be an architect (Saint, 1983, p.161).

Within this thesis the 'image' of the architect refers to its symbolism, it refers to a simplified description which encompasses a multifaceted identity (Pallasmaa, 2011). The thesis contends that a gap between the image and the actuality of architectural practice becomes apparent during the latter part of the twentieth century (Cuff, 1991; Crawford, 1991; Schneider \& Till, 2009).

\section{Context}

The second premise of this thesis is the effect of context as a catalyst for the architect's evolving image; its role in the formation of the gap between this image and the actuality of practice, but also how changing conditions challenge the architect's role:

...crisis keeps cropping up. But unless architecture realises that calculated uncertainty is one of the great generators for what it should be doing, then I think the profession has no future. But I think architecture has. (Price, 1975/2017, p.240)

Within this thesis 'Context' refers to the circumstances within which architects are working. The thesis explores how the image of the architect has been constructed as a pattern of responses to complex and interwoven social, political and economic developments (Blau, 1984; Symes et al., 1995; Burr \& Jones, 2010; Carrio, 2015). The thesis contends that the evolving context leads to tensions and challenges the architect's role.

\section{Negotiation}

The third premise of this thesis identifies that architects offer a wide range of skills and how they deploy these skills enables them to respond to the evolving context, demonstrating an adaptive identity, able to negotiate challenges to the role: 
...architectural know-how can be deployed in multiple ways and on multiple contexts that exceed, but of course do not exclude, the design of buildings (Schneider \& Till, 2009, p.108).

Within this thesis 'negotiation' is a transitional term, referring to how architects manage these challenges to reach a favourable outcome (Kapp, Baltazar, \& Morado, 2008; Hill, 2012; Awan et al., 2013; Samuel, 2018). The research therefore, contends that focusing on critical skills linked to this adaptive nature, rather than an incongruous image, might enable architects to continue to evolve the role.

Having identified that the motivation for undertaking the study is to understand how the role of the architect is changing and that this investigation is undertaken through the themes of image, context and negotiation. The next section sets out the scope and aim of the thesis, together with how the three themes are delimited.

\subsection{AIM AND SCOPE OF THE THESIS}

The discussions in sections 1.2 and 1.3 provide the background for the aim of this thesis, which is to identify how the architect's role is changing. The aim is explored by investigating the effect of tensions and challenges on the architect's image, caused by an evolving context. The field of research outlined here is broad and therefore to investigate the themes within the scope of this thesis, delimitations have been set. The purpose of this section, therefore, is to explain the scope of the study as well as the limits within which the research takes place. Although a key theme of the study is the image of the architect, the exploration is concerned with architectural culture (Stevens, 1998; Till, 2009) and how it is "conditioned by the broader political-economic context in which it is commissioned, designed and understood" (Jones, 2009, p.2519), to identify which aspects of the image are incongruous within the contemporary context. An in-depth exploration of the ways in which practices promote their work will not be attempted within the scope of this thesis, however the research recognises that the methods architects use to publicise their work have the effect of reinforcing the image of the architect, a subject which is touched on in the research section chapter 4. Likewise, the role pedagogy plays in reinforcing the image is touched upon but not explored in-depth. A further edge to this research is its focus on the context of the Anglosphere, chiefly the United Kingdom, United States of America, Australia and New Zealand. Finally, it is important to reiterate that the focus of this research is not concerned with the discipline of architecture, but the role of the architect. 
Within these described boundaries there are additional foci as the existing framework of literature sits across the fields of the humanities as well as architectural theory and includes identity work, cultural as well as feminist studies. This thesis balances a critical approach to the existing theory and an empirical study of practitioners, to bridge a gap in the existing body of knowledge surrounding the future role of the architect. The theoretical analysis explores two lines of inquiry. First, analysis is undertaken, contained specifically to the key historical moments, which explicate how the layering of identities attached to the contemporary image occurred in response to changing context. Second, the themes surrounding tension and challenge concentrate on issues which directly challenge the image of the architect such as ideas of autonomy and elitism and the exploration of negotiation flows from these themes. The third area of research occurs through a qualitative study, the purpose of which is to interrogate the theoretical discussions in greater depth from the experiences of architects working in practice. The scope and methodology of this part of the thesis are outlined in the following section 1.5. 


\subsection{RESEARCH APPROACH}

In consideration of the aim and motivation for the study, as outlined in the preceding sections of this introduction, this section discusses the research approach, or methodology, the study has taken to investigate the key themes of image, context and negotiation. It also summarises the methods for data collection, verification and analysis and ethical considerations the research has followed.

\section{Research Strategy and Design}

The foundation work for this research commenced with a critical review of the key themes, to position this study theoretically. The results of this aspect of the research are outlined in the literature review, chapters 2 and 3, which investigate the image of the architect, placing it within a historical context, the emergent challenges to the architects' role and theories surrounding the future reimagining of the profession. Undertaking a systematic exploration of the key sources, theories, issues and debates around the topic, and interrogating the main questions and problems addressed in the current literature (Groat \& Wang, 2002), established the topic's relevance, located the thesis within the existing literature, and fed into the framework of the qualitative part of the research.

A qualitative method was adopted as the preferred means of data collection and was considered appropriate to this research as a way of studying, “...first-hand encounters with a specific context. It involves gaining an understanding of how people in real-world situations 'make sense' of their environment” (Groat \& Wang, 2002, p179). Additionally, the techniques used within qualitative methods afford flexibility to explore the key themes in depth with the participants, to gain clarity or more detailed information (Johnson \& Turner, 2003). Providing rich data is described by Geertz as "thick description" (Geertz, as cited in Groat \& Wang, 2002, p.74) whereby detailed information gathered helps to contextualise a study. This notion is explored further by Richards (1999) who states that there are four considerations when categorising data as 'rich' they are "relevance, impact, complexity, and fluidity" (Richards, 1999, p.414). The interviews were therefore structured to facilitate open discussion and additional observations from respondents were encouraged. The research did not follow a pure qualitative method, however, which is categorised by Johnson and Turner (2003) as open-ended and freeflowing. The interviews, instead, followed a format of subject areas, using an interview guide, constituting a form of directed conversations, thus, finding a balance between a loose, exploratory and unstructured approach and the more rigid structure of orchestrated 
questions. Patton (2015) explains that the advantage of an interview guide is to provide a general framework of the themes to be discussed, to ensure a point of comparison between respondents, whilst facilitating an open exploration.

The qualitative research took place halfway through the programme for this study so that sufficiently detailed data could be collected, and to enable a process of reevaluation of the topic of inquiry. Bogdan and Biklen (2007) characterise qualitative research as inductive, with the theory emerging "from the bottom up" (Bogdan \& Biklen, 2007 , p. 6). A holistic re-evaluation of the key issues, therefore, emerged following the qualitative aspect of this research, in the concluding stages of the study.

\section{Cross-National Study}

As outlined in the research strategy section, the objective of the qualitative research is to investigate architects' experiences, attitudes and actions in response to changes in their role. There are two arguments for conducting this research as a cross-national study: first as a test of objectivity and second as a means of testing the wider relevance of the study (Hantrais, 1999). Caven, Navarro-Astor and Diop (2012) observe that a cross-national study reveals how behaviour is affected by the social context and therefore research into the architectural profession “... must acknowledge the different social, cultural and political systems in place" (Caven et al., 2012, p.368). As a practicing architect in the United Kingdom, the researcher has acquired a detailed knowledge and understanding of the issues surrounding the topic of inquiry within the British context. This insider's knowledge provides advantages to the research, particularly through an understanding of the issues raised by interviewees, however, as outlined by Hantrais, there is the possibility that the researcher's experience also directs the focus of the study through a biased lens.

Hantrais (1999) follows the developments in cross-national comparative research, observing that such studies can derive from "concern to observe social phenomena across nations, to develop robust explanations of similarities or differences" (Hantrais, 1999, p.93). Hantrais guides selecting effective frames of reference, recognising particular aspects of society to be particularly relevant factors such as legal and administrative structures, industrial policy and the impact of international trends on economic systems. New Zealand and the United Kingdom share comparable, although not identical, pedagogical and practice-based cultures in architecture, however, the researcher has no direct experience of working in New Zealand. By also examining the topic of inquiry within the New Zealand context, the objectivity of the research can be tested. 
Additionally, any common patterns that focus on individuals' experience, rather than generalised national traits, can be identified, thus, broadening the relevance of the study. The issue of bias is discussed in additional detail later in this section (1.5)

\section{Sample Design}

A key purpose of the study is to gain insight into the affect of architects' changing roles, by discussing the phenomenon with the individuals involved. Part of the research strategy considers sample design to ensure the correct balance of detailed and comparative data is collected. Groat and Wang (2002) outline how a purposive sample might discover detailed patterns of information from a particular group of people. The objective of this qualitative study is not to provide a generalised view, but rather, "qualitative methods are often used in evaluations because they tell the program's story by capturing and communicating the participants' stories" (Patton, 2015, p.10). Thus, through the study of a select group of individuals, the participants' experiences and responses to the themes will provide insight into how a particular group of architects negotiate the challenges they face in today's building industry. Clues can then be taken from the findings, to feed into a broader discussion.

Sandelowski (1995) observes that judgement is key to determining the sample size in qualitative research, insufficient interviews will fail to achieve "new and richly textured understanding of experience" whereas too many interviews will put at risk "the deep, case-oriented analysis that is a hallmark of all qualitative inquiry" (Sandelowski, 1995, p.183). For this reason, Onwuegbuzie and Collins (2007) propose a framework for developing sample designs in mixed method approaches to research, based on the objective of the research. Onwuegbuzie and Collins (2007) cite Creswell, Morse and Guest, Bunce and Johnson to identify that phenomenological and interview-based research tends to be located within a sample size range of less than 6 and up to 12 interviews. In consideration of the need to balance variety in the sample and rich data collection, ten architects were approached in total, five from New Zealand and five from the United Kingdom, with eight (four from each country) choosing to participate. 


\section{Selection of Participants}

The choice of participants was structured around a matrix ${ }^{1}$, which was prepared to draw from a range of experiences of the actuality of architectural practice, most broadly, within the limits of this thesis. Architects selected for interview included representatives from well established, second-generation firms; medium to large offices; small to mid-sized offices as well as single practitioners (RIBA 2018). Participants from each of these four types of office were selected from both New Zealand and the United Kingdom.

Purposive sampling is used as a way of focusing research on a particular group of people which share traits pertinent to the topic of inquiry (Groat \& Wang, 2002; Patton, 2015; Etikan, Musa \& Alkassim, 2016), therefore, participants were also selected based on particular characteristics. The first shared characteristic between participants is that they all work in design-focused practices. Design architects were chosen so that challenges to the creative aspects of the architect's role can be most closely observed. The second shared characteristic between participants relates to career stage. Using the terms Cuff (1991) uses within her study of practice culture, seven of the eight architects could be categorised as 'full-fledged' with the eighth being “middle years” (Cuff, 1991, p.148) therefore all have achieved grades of seniority within the profession. There are three reasons for choosing architects at this stage of their career: first, to focus discussions on the role of the architect exclusive of participants' attitudes to the internal hierarchies of practice. Second, to gather observations from those with a range and similar lengths of experience and third, to understand responses to the challenges by those with strategic responsibilities. The selection of participants used homogenous purposive sampling (Etikan et al., 2016) with design focus and seniority providing the connecting characteristics between participants. Taking this approach to the sample design, facilitated the collection of a broad range of experiences within a clear structure, thus, focusing the research to suit the scope of this thesis.

\footnotetext{
${ }^{1}$ Refer to vi. Appendix for selection matrix.
} 


\section{Data Collection}

As the Research Strategy and Design section has described, questions and ideas arising from the literature review fed into the qualitative study. The subject areas were not prescriptive, rather areas of discussion framed around the three key themes; image, context and negotiation. These were framed as an exploration into the continuing role of the architect in the building industry and questions were categorised as follows:

1. Questions which established the perceived differences between the image and the nature of architectural practice.

2. Questions which ascertained practitioners' experience of and response to changing conditions.

3. Discussion of strategies practitioners use to counter or adapt to new and emerging conditions.

Sample questions around these categories were provided within the interview guide, and these were sent to participants in advance of the interview to enable them to reflect fully on the issues prior to the meeting. A copy of this document can be referred to in vii Appendix. The interview guide was not a definitive list of the questions asked during the interviews; additional questions grew from the discussions and often, due to the fluid manner of the discussions, answers overlapped naturally. Therefore, the interview guide provided a coherent structure, or datum, for ensuring a consistent approach to the interviews. Further, the open and flexible nature of the semi-structured interviews were valuable in establishing a rapport between the interviewer and participants and led to detailed and rich data collection.

Participants were first contacted by email to canvass their interest in contributing to the research. Those who responded positively to the initial approach were then sent a letter of introduction which outlined the details of the study incorporating the interview guide, the interview procedure, proposed methods of recording the data and measures to protect participants' confidentiality. The interviews with architects based in New Zealand were conducted at a place of their choosing, either in their offices or a café. Although the intention was to interview all participants in this manner, time and cost considerations meant that the architects based in the United Kingdom were interviewed via video conferencing. 
Research into the validity of online methods of interviewing is a growing field. In qualitative research face-to-face techniques are to be preferred, as with video interviews, "the interviewer will tend to lose some of the immediacy of the face-to-face encounter, and there may also be technical difficulties to overcome" (Coleman, 2012, p.254). Nevertheless, within the growing options for remote interviewing, video conferencing offers greater engagement than audio, telephone or asynchronous interviewing which, in the absence of visual clues, present a challenge to building rapport and provide a greater opportunity for misunderstanding (Coleman, 2012; Deakin \& Wakefield, 2014). Further, with an increasing number of international studies being undertaken, online interviews are increasingly used in social sciences and are regarded with increasing validity as a qualitative research technique (Deakin \& Wakefield, 2014). Obstacles with regards to building rapport or effects on visual clues were not apparent in the data collected through this research. All respondents spoke candidly and fluently on the issues and the interviews conducted by video conferencing did not appear less frank or more constrained by the online medium than those conducted face-to-face. Therefore, although face-to-face interviews would be preferable for all interviews, considering the constraints of the study, video conferencing represented an acceptable alternative. For the remote interviews, Skype was chosen as a reliable platform and the most readily available to all participants.

With the prior agreement of participants, face-to-face interviews were recorded with a digital audio device and the Skype interviews were video recorded within the application. Observational notes were also gathered by the researcher during the interviews; these included follow up questions as prompts to explore points made during the conversation and reflections as to the content of the discussion. After each interview, a summary of the researcher's initial observations on the content and tone of the discussion and a record of the notes taken during the interview was prepared. These supplied a supplementary source of information to assist the transcription process.

\section{Analysis}

The eight interviews were transcribed by the researcher using transcription software to produce a first draft, then the final documents were re-analysed against the recordings and refined "...taking into consideration, and balancing, four overlapping transcription issues: readability, granularity, accuracy, and research agenda" (Jenks, 2013, p.255). Using Jenks' considerations, the transcriptions were used primarily as raw data to form the 
arguments pertinent to the aim of the research. Their secondary purpose was evidential; providing direct quotes or paraphrased concepts, which are threaded throughout the research chapter to illustrate the arguments. With this in mind, superfluous information was kept within the scripts but minimised and granularity, or the complexity and stutterings of spoken discourse, were removed to ensure clarity within the quotes used. Poland (2003) acknowledges the incongruity between the spoken and written word, observing that "verbatim quotes make for difficult reading" (Poland, 2003, p.272) however, advises that this process of refinement should be undertaken only after the analysis has taken place. The method of transcription, undertaken directly by the researcher, ensured that this sequence was maintained, and that care was taken not to alter the tone or meaning of the quotes used.

Following the transcription process, thematic analysis was undertaken using a template (King, 2004). The initial coding template was prepared, referencing the themes which emerged from the literature review (chapters 2 and 3) and the interview schedule. The themes were sub-coded into categories according to the three key themes of image, context and negotiation. Following a provisional reading of the transcripts, additional themes and sub-themes were incorporated into the template to form a draft structure for the research section (chapter 4). Following this preparatory work, relevant sections of the transcripts were organised within the draft structure, to observe overlapping ideas and correlations. A final template was prepared, after which writing of the research chapter commenced alongside continual reflection on the relevance and clarity of the findings to the research aim and scope of the thesis.

\section{Validity and Reliability}

Validity refers to whether the research precisely fulfils the purpose of the investigation, whereas reliability refers to consistency and whether a measurement taken under the same conditions can be repeated (Bush, 2012). Bush observes that validity and reliability are often at odds with each other and offers that in qualitative studies validity should take priority. In discarding the importance of absolute knowledge through research, Kvale (2007) also perceives the quality of knowledge becomes the priority and suggests this can be achieved through "continually checking, questioning and theoretically interpreting the findings" (Kvale, 2007, p123). Morse, Barrett, Mayan, Olson and Spiers (2002) observe that focusing on strategies for establishing trustworthiness at the end of a study rather than through the investigation creates possibilities for validity and reliability concerns 
arising at a point which is too late to rectify. Constructive, or in-process, techniques require neutrality, consistency, applicability and "truth value" (Morse et al., 2002). This position accords with Kvale and means ensuring coherence between the research question and the research strategy; an appropriate sample; collecting and analysing the data concurrently and thinking theoretically to feed new ideas which emerge from the data back into the research process. Verification within qualitative research is therefore iterative, concerning "congruence among question formulation, literature, recruitment, data collection strategies, and analysis" (Morse et al., 2002, p. 17).

In consideration of the measures outlined by Morse et al. (2002), Kvale (2007) and Bush (2012), the research approach which has been outlined in this chapter demonstrates the processes undertaken to verify the quality of data received. In particular, the Data Collection and Analysis sections describe the layered process undertaken through note taking, review and transcription of the interviews as a means of checking and appraising the data for consistency and coherence. Additionally, the thematic analysis demonstrates an iterative process of checking, cross-referencing, comparing and affirming the findings against the research aim. The Research Strategy and Design section also explains how a holistic re-evaluation of the key issues emerged following the qualitative aspect of this research. This process of auditing the data was undertaken as an exercise in reflexivity (Seale, 1999) to ensure the ideas and themes emerging from the research fed into the process but also remained germane to the topic of inquiry.

While the measures described in the preceding paragraph ensured the validity of the research, the reliability of the findings was also considered. Seale (1999) re-frames reliability as credibility and proposes member checks as a means of establishing this. The review and transcription process outlined in the Analysis section was also an exercise in critically appraising the findings to ensure consistency in the information, as well as checking for the researcher's misinterpretation or bias. Patton (2015) advises testing for consistency across interviews by immediately scrutinising the data, making notes and seeking clarification from participants. These measures were undertaken both within the interviews, where unclear meanings and statements were clarified by the researcher repeating the statement back to the participant. Outside of the interviews, open communication was maintained between the researcher and participants so that clarifications could be sought if necessary, transcripts were offered for review to the participants. Two of the participants took up the offer and one provided additional clarifications. 


\section{Ethics}

The research strategy and design necessitated detailed engagement with individuals. The focus of the study relates to how architects approach their working environment; therefore, interviews are concerned only with specifics of the subjects' professional life. No personal harm or risk was anticipated towards the interviewees; however, ethical considerations were associated with this approach. Given the participants were asked to share details about their experiences and attitudes to a range of aspects related to their professional lives, including business strategies and client relationships, ethical considerations were addressed as follows.

First, due consideration of the University's ethical research standards was taken, including obtaining permission from Victoria University of Wellington's Human Ethics Committee (HEC) ethics \#26016. Second, any potential risk or harm to the subjects arising from conversations regarding their experiences at work was addressed. The participants' identities have been kept confidential by the researcher by aggregating the data, and identifiable information has been removed. Additionally, a code has been given to each participant; the details of this is outlined in footnote ${ }^{4}$ in Chapter 4. Third, informed consent was obtained from the participants and in compliance with HEC approval \#26016. In advance of the interviews, each participant received a detailed information sheet outlining the nature and purpose of the study; the way that data will be collected and how that data will be used for academic purposes, and details of confidentiality measures taken for the use of the research data (including all correspondence between parties). Informed consent forms were included for signing prior to the interviews' commencement.

\section{Limitations and bias}

As outlined in this methodology section, the researcher has undertaken this investigation with the aim of maximising validity. However, as a qualitative study, potential limitations should be noted; these considerations are addressed here.

First, it should be noted that this investigation has undertaken a single method approach to the research. Bush (2012) argues that triangulation may be "incompatible with certain approaches to qualitative research that value and celebrate individual or subjective ways of seeing and interpreting phenomena.” (Bush, 2012, p.86). Certainly, the aim of the research in this thesis is to explore individual architects' experiences and 
attitudes to their working context, therefore is by nature subjective. Nevertheless, mixed method approaches are considered to offer greater verification of the facts (Bogdan \& Biklen, 2007; Patton, 2015). The researcher acknowledges this potential limitation in the research but argues that dependability has been sought firstly through the constructive processes undertaken, through the depth and complexity of the data gathered and the methods of cross-checking and reflection outlined in the preceding V alidity and Reliability section.

Second, the sample size could be considered to provide a narrow body of information from which to test the topic of inquiry. The purposive sampling approach, including decisions over the sample size, has been explained in detail within the Sample Design aspect of this methodology section. The sample size is considered to balance the requirement for rich data with the scope of the thesis, however, the significance of the findings should be understood within the context of architects working in design-focused practices. Consequently, although the research explicates the phenomena within this specific group, the results may not be comparable with practices working in different areas of the discipline, such as technical delivery practices or multi-disciplinary firms.

The limitations discussed above will be addressed in greater detail in the concluding discussions where opportunities for further research strands are explored. A final potential for bias stems from the researcher's previous architectural career. Lupton (2000) suggests that in the case of an interviewer and participant having a shared identity, the interviewer may advance particular views or interpret the data through a biased lens. An additional consideration is three of the participants were known to the researcher; although it is difficult to ascertain what effect this had on the responses. There is the possibility that participants were more reserved in their responses, or conversely may have been more candid in their answers than to a stranger. The researcher addressed these considerations by making questions as open as possible and keeping the wording neutral to ensure no value judgements were inferred by the interviewer during the conversations. Additionally, the control of a cross-national study enabled a reasonable comparison in the quality and detail of data received and, thus, establishes that prior knowledge of some of the participants did not appear to influence the manner of the interview.

This section 1.5 has outlined the methodology adopted by this research; the next and final section of this introductory chapter sets out the structure through which the research will be presented. 


\subsection{OVERVIEW OF THE THESIS}

As this introductory chapter has outlined, this thesis is constructed as a study of the effects of changing context on the image of the architect and examines how the role might continue to change to ensure its future significance. The thesis is structured around five chapters.

Following this introduction, Chapter 2 commences the first part of the literature review, providing an analysis of the literature and theory surrounding the development of the architect's image. This chapter commences with the first known historical description of the architect by Vitruvius and proceeds to observe the layering of identities through the Renaissance, Enlightenment, Industrial Revolution and the modern movement. Chapter 2 observes how the image of the architect is linked to key shifts in the political and socio-economic context, giving clues about the adaptive nature of the profession. The chapter also introduces ideas which will be explored in Chapter 3, whereby aspects of the image have implications for the future development of the profession.

Flowing from the exploration into the architect's image, Chapter 3 completes the literature review by analysing the contemporary challenges to architectural practice and theoretical approaches to negotiating these. This chapter commences with an exploration of key texts which identify a tension between the image of the architect and the actuality of practice; identifying aspects of architectural culture as catalysts for this tension. The chapter also explores the effects of changing context on the role of the architect, discussing ideas of fragmentation, compromise, undermining and value. The second part of Chapter 3 reviews literature which proposes how architects might negotiate these challenges, with notions of diversification, specialism and critical practice considered as methods of response. Chapters 2 and 3 direct the content of the qualitative research which follows in Chapter 4.

Chapter 4 presents the results of the qualitative research, undertaken following the methodology described in section 1.5 of this introduction. The chapter examines architects' experiences of issues surrounding the three key themes: image, context and negotiation. First, the architects' motivations for entering the profession are discussed, then their experiences of the tension between the image of the architect and the actuality of practice. There then follows an exploration of the challenges the architects face and how they negotiate these, presented under the corresponding themes laid out in chapter 3. The research discusses how the image of the architect is less important to the 
participants than the process of designing, leading this section to conclude that the findings are important in discussing how the architect's role might evolve.

The thesis concludes with Chapter 5, which summarises the previous chapters, describing their implications and how they feed into the research. The concluding discussion considers how this investigation might be built on to increase the scope and therefore widen its relevance, as well as suggesting further strands of investigation which could grow from this research. 


\section{CHAPTER 2}

\section{Constructing the Image of the Architect}

\subsection{INTRODUCTION}

The introduction to this thesis outlines that its objective to explore how the architect's role is changing. The literature review compiles a framework of contemporary theories that identify and explicate the increasing gap between the image of the architect and the actuality of architectural practice (Cuff, 1991; Johnson, 1994; Till, 2009; Samuel, 2018). This chapter tracks the construction of the architect's image to provide a comparative foundation for the following Chapters 3 and 4, which examine the actuality of practice. Although this chapter undertakes a historical narrative, it does not attempt to cover the vast historical tradition in architecture, but rather provides vignettes of key moments where the image has changed. Further, this is not a discussion about architectural theory over the ages, but how circumstances and theories are responsive to each other, and as such, how the formation of the architect's image is a product of this interaction.

Following on from this introduction, this chapter is structured into five further sections; first, section 2.2 examines the role of Vitruvius in the formation of the architect's image, drawing on the work of McEwen (2003) and Till (2009). Agrest (2000); Vesely (2004) and Perez-Gomez (2005) are referred to in section 2.3 which commences with a brief overview of the Gothic period before observing the developing image of the architect as an artist in the Renaissance. Next, autonomy in the Enlightenment is discussed in section 2.4, where Habermas (1983/2005); Nesbitt (1995) and Vesely (2004) are particularly informative. Section 2.5 examines how the Industrial Revolution triggered the emergence of the professions and the image of the gentleman architect, which is covered in particular depth by Larson (1977); Cuff (1991); Crinson and Lubbock (1994) and Stevens (1998). Finally, section 2.6 explores how the contemporary image of the architect was crystallised during the modern period drawing, in particular, on the work of Jencks (1973); Harvey (1990); Heynen (1999) and Huxtable (2008). The study of the architect's image will conclude in section 2.7 by observing how the image of the architect has evolved historically as a layering of identities, but also summarises how the public response to the modern movement instigated further change in the architect's role. This 
leads the discussion towards the contemporary context and the following Chapter 3 whereby tension between the image of the architect and the actuality of practice will be explored.

\subsection{DEFINING AN ARCHITECT}

The image of an "Architect" as a distinct occupation is a relatively recent phenomenon. Through the ages, the responsibility for the design of a building has been ascribed either to an exceptional individual of learning with aptitudes crossing several spheres (Kostof, 1977; Crinson \& Lubbock, 1994) or a network of designer/craftspeople organised around a common ideology (Rudofsky, 1964). Nevertheless, architecture communicates complex ideas via the concept of image, "We are usually affected only by the surface message and ignore the unintentional unconscious messages, but they are the most significant ingredient in a work of art" (Pallasmaa, 2011, p51). Consequently, the earliest aesthetic architecture is linked to authority and power (Hirst, 2005). The architecture of the Roman Empire was ubiquitous and can, therefore, be seen as instrumental in constructing and reinforcing the image of Empire, creating a sense of unity under a common architectural language.

The earliest surviving text specifically describing the architectural discipline is acknowledged to be Vitruvius's De Architectura (circa 30BC). This provides an early, tangible, definition of the role of an architect and dates from the period when Rome transitioned from republic to empire (McEwen, 2003). McEwen describes Vitruvius'

ambition in dedicating De Architectura to his patron Emperor Augustus, when he asserts "I will without a doubt prove myself possessed of the greatest authority - not only for those who intend to build, but also for all learned men" (Vitruvius, Rowland \& Howe,1999, p.23). Indeed, Vitruvius' influence in defining the image of the architect is evidenced by the texts' continuance. The ethics and principles laid down by Vitruvius informed the architectural rulebook through the Renaissance and continued to act as the founding principle to which all architectural theory referred until the mid $18^{\text {th }}$ century (Stevens, 1998). Vitruvius' work still “...forms the knowledge repository of our field, regularly aired in Architecture 101 modules across the globe..." (Samuel, 2018, p.52) and based on this enduring influence, has set a staunch narrative for the image of the architect from an early time. 
Vitruvius' treatise is wide-ranging in its scope, but most significant to this thesis, it “... spelled out the tremendous range of knowledge an architect needs" (Cuff, 1991, p.84). Vitruvius' precepts on how architects should be educated include, amongst other skills, wide knowledge of history and philosophy, music, theatre, medicine, law and astronomy (Vitruvius et al., 1999). Vitruvius, therefore, describes the image of the architect as polymath: an exceptional and knowledgeable individual. Although architectural texts preceding Vitruvius are no longer in existence, it is understood that these were simply technical references, therefore, the rhetoric presented through Vitruvius' writing suggests its key purpose was not so much to “...write a technical manual” rather “... a polemical document” (Patterson, 1997, p.359). Vitruvius' writing is couched in lofty terms that accentuate his view of the prodigious nature of the discipline and the unique skill of those that undertake it. Regarding the description of the role as made in De Architectura, Vitruvius might simply be understood to be penning a celebration of a complex and skilful discipline, yet "Vitruvius seals his relationship to his patron with repeated written invocations of "Imperator" and "Caesar" he turns theirs into a permanent, indissoluble connection." (McEwen, 2003, p.129). Vitruvius' ambition can, therefore, be seen to extend beyond capturing the principles of architecture.

Further, Till identifies the contemporary architect's mission to rationalise the disorderly descends from the ten books and observes that:

Here we have the first conflation of the value of profession, practice, and product that is to be repeated throughout architectural history: a prescription of order that applies equally to the knowledge of the profession, the structure of practice, and the appearance of buildings. (Till, 2009, p.28).

Thus, Till acknowledges Vitruvius' influence, suggesting that the desire for architectural order stems from Vitruvius' teaching and concludes that his tenets on proportion present architecture as a discipline of control (Till, 2009). In appealing to the Emperor's imperial objectives, "it was not architecture as such that initially attached Vitruvius to Julius Caesar's might. It was, rather, the connection of architecture to imperium" (McEwen, 2003, p.38). Through Vitruvius we can, therefore, observe how the architect's role was defined as possessing wide-ranging and intellectual skills, (Cuff, 1991; Samuel, 2018), but also reveals an ambition to act in the service of authority, framing the role in response to political change (McEwen, 2003; Till, 2009). Thus, the image of the architect as defined by Vitruvius is a polymath with aspirations for status. 


\subsection{THE RENAISSANCE ARTIST.}

Following the fall of the Roman Empire, the relationship between architecture and power continued, with the Church, Kings and Feudal Lords replacing the Empire. This ruling elite became the new patrons of the arts (Cuff 1991). Hickson and Thompson (1991), Epstein (1998), Gelderblom \& Grafe (2010) and Carrio (2015) trace the history and role of guilds in mediaeval times (and within that tradition, craft guilds) which emerged as a pragmatic collective response to expansive foreign trade within Byzantium-supported Italian coastal towns. Boyd and Danks (2000) and Carrio (2015) describe how traditions of knowledge, skills and ethics would be handed down through apprentice structures. The development of artisan organisations was, thus, organic and pragmatically responsive to political and economic circumstances. During the Gothic period, such craftsmen produced great architecture; at this time, designers and builders are synonymous (Gutman, 1988; Wiscombe, 2006). Craftsmen operated under the umbrella of the guilds and in the service of a commissioning elite. The architect's identity (if it existed at all) was entirely invisible, with buildings seeming to emerge out of the endeavour of a series of artisans, each tasked with crafting a part of the whole (Pérez-Gomez, 2005). In the absence of an apparent master-planner, the patron acquired the role of the creator, as the entity most closely associated with the completed architecture (Larson, 1977; Stevens, 1998).

The role of the architect is, then, indiscernible until Florentine cultural politics during the Renaissance shaped the status of artists and defined the intersection between theory and practice (Cuff, 2012). Padgett \& Ansell (1993), in their essay on the consolidation of power under the Medici, explain how critical Cosimo de Medici was, as a principal patron to the intellectual and artistic developments in the Renaissance. The Academia del Disegno, under the direction of Vasari, gained recognition as an institution through Cosimo de Medici, who was its nominal head (Hughes, 1986). Cuff describes how the "... disegno signified the intersection of intellect and creation, its artists exhibiting more than craft by virtue of schooling in the collected works and ideas of the visual arts" (Cuff, 2012, p.385). Thus, there was a reimagining of how artists learned, by focusing as much on theoretical education as on practical application (Cuff, 2012).

A key figure in theoretical development in the Renaissance, was Leon Battista Alberti. In the fifteenth century Alberti's Ten Books, a treatise on composition, marks the point that Vitruvius's rulebook was rediscovered (Vesely, 2002). Reflecting on Vitruvius' list of skills, Alberti represented "the paragon of the nomo universal" (Beck, 1989, p.9) 
being widely skilled in a range of subjects, he "in short, mastered all the traditional arts of the medieval courtier and all the new ones of the Renaissance intellectual" (Grafton, 2002, p.10). Agrest (2000) identifies Alberti as being instrumental in defining architecture within the body of the liberal, rather than mechanical arts. The Renaissance period saw development in modes of representation, in mathematically constructed perspective (Agrest, 2000; Vesely, 2004; Till, 2009) and of 'the classical' in architecture (Eisenman, 1984).

During this time the architect emerged as an identifiable individual, but the image of the architect was considered as part of a triptych with artist and sculptor. Thus, architecture was undertaken by people skilled in many areas and to which architecture was a secondary pursuit (Crinson \& Lubbock, 1994). Alberti was not the only architectural polymath; “...Brunelleschi was a goldsmith by training... and Michelangelo was a painter and sculptor" (Crinson \& Lubbock, 1994, p.3). Within this context, Renaissance architects differed from the master builder of the gothic period; with an emphasis on artistry, the architect eschewed the more pragmatic engineering and building tasks (Wiscombe, 2006). A key development to cement this shift in the architect from a practical to artistic focus, was the exploration of architectural drawing. Agrest (2000), Vesely (2004), Perez-Gomez (2005) and Till (2009) describe the significance of architectural representation in separating the architect as an artist from the craftsman. Expertise in both mathematics and art was fundamental to developing evolutionary modes of representation through mathematically constructed perspective. Thus, in the Renaissance, the image of the architect as a polymath developed towards the architect as an artist.

Nevertheless, architectural development in the Renaissance period was reflective of principles of an "already valued architecture" (Eisenman, 1984, p.156). The mathematical developments in architecture during this period were, therefore, concerned with the representation of a traditional form of design rather than scientific exploration of 'universal reality' (Vesely, 2004, p.293). Therefore, although the role of architect and architectural representation evolved over the Renaissance period, the image of architect as a distinct discipline developed little from the point in which De Architectura set out the architect's image in homage to the imperial idea. 


\subsection{ENLIGHTENMENT AND AUTONOMY}

Scientific revolution leads sixteenth century Renaissance thinkers towards the seventeenth century and the Enlightenment (Bristow, 2017). Copernicus, Kepler and Galileo's heliocentric theory challenged the established conception of the "sublime spectacle of the starry sky, that the ancients thought to be a perfect sphere" (Harries, 2002, p.152). Thus, scientific development during the Enlightenment, principally Newton's Principia Mathematica (1687), demonstrates how the sun is at the centre of a system of planets (Wilson, 1989). This advance is significant in transforming an established understanding of the earth and its place within the cosmos and it is at this point that people begin to look towards science rather than religion to explain the natural world. The scientific understanding leads to questions over the autocratic power of church and state (Bristow, 2017). For the first time in modern European history, human thought was directed to challenge tradition and create new principles of theory and practice. This section will explain how, through a release from tradition and established power structures, the Enlightenment gives rise to such ideas as autonomy, subjectivity and the concept of genius. The development of the architect's image will be shown to grow from these ideas, towards independent thought and creative design processes.

In explaining theoretical development towards autonomy, philosopher and sociologist, Jürgen Habermas writes extensively about the impact of German Enlightenment thinkers on the structure of intellectual disciplines. Habermas (1983/2005) points out that before the Enlightenment, religion and metaphysics were interwoven, with art developed as an expression of this holistic arrangement. He refers to Weber's idea of modern culture as the point that the core ideas in this unified model of theology and metaphysics are split "into three autonomous spheres. They are: science, morality, and art" (Habermas, 1983/2005, p.168). Habermas describes how the belief in unlocking the intellectual potential of each of these distinct disciplines could effect a dominion over nature and enable understanding of "the world and of the self, moral progress, the justice of institutions, and even the happiness of human beings" (Habermas, 1983/2005, p.168). Habermas, therefore, considers that Enlightenment thinking challenged tradition and authority by dismantling the universality lauded during the Renaissance. Vesely (2004) offers an alternative position to Habermas' interpretation of Weber and Kant, suggesting that the 'three autonomous spheres' of theology, science and art continue to be linked, undertaking a transformative correlation rather than a distinct and immediate separation. Vesely identifies that tension arises from splitting the 
disciplines because in architecture "it is impossible to separate the fine arts and the practical arts," (Vesely, 2004, p257). Vesely's analysis that architecture straddles two spheres, reinforces the image of the architectural polymath.

Kantian philosophy is central to the development of autonomy in art and, therefore, also in the image of the autonomous architect (Rawes, 2007; Gough, 2013). Rawes (2007) argues that reflexive thought, as advocated by Kant, illustrates the architect's ability to use technical and aesthetic judgement. Rawes considers reflexivity to be more important to the design process than reason and knowledge, as it elicits imagination. Similarly, Gadamer (1975/2013) raises the concept of "aesthetic consciousness" (Gadamer, 1975/2004, p.74). Using Kant's definition, he explains that aesthetic means the judgement of taste "Kant had already accorded taste the significance of a transition from sensory pleasure to moral feeling” (Gadamer, 1975/2013, p78). Reflexivity, judgement and taste are ideas that rely on subjectivity, "the sublime only requires that the reader or listener have conceptual range, taste, and the ability to sense what everyone senses first" (Lyotard, 1985, p.7).

When considering this image of the architect, a key question emerges as to how creativity and subjectivity become manifest through architectural expression. During the Enlightenment, the act of drawing on established design traditions to achieve a contained and balanced harmony is set aside to pursue a new expression that seeks to evoke a visceral response through the sublime. Lyotard (1985), Nesbitt (1995) and Rawes (2007) discuss the significance of the sublime, describing it the power to astonish. Nesbitt describes the sublime as referring to "immense ideas like space, time, death, and the divine" (Nesbitt, 1995, p.98). She studies the aesthetic discourse during the Enlightenment as a contest between the sublime and the beautiful. Through the sublime, Nesbitt concludes that Enlightenment architecture can be seen to overcome nature, indicating that beauty can be seen to derive from nature, whereas the sublime is created in the mind of the human. Thus, the pursuit of the sublime during the Enlightenment is an exercise in subjectivity, requiring intellectual emancipation, and can be seen to contribute to the image of the architect as autonomous.

Autonomous art is created within the mind of a human; it requires invention and creativity, "identified with the qualities of exceptional individuals" (Vesely, 2004, p.262). In other words, to capture the sublime without reference to the past, free of the constraint of theological tradition, autonomous art and, therefore, architecture becomes the production of genius (Lyotard, 1985; Vesely, 2004; Gadamer, 1975/2004). Thus, 
reflexivity and subjectivity, which are drawn from Kant's philosophy, give rise to the image of the architect as an autonomous genius

Given the ambitions of Enlightenment architects are centred on subjective and visceral themes, how the sublime is communicated becomes significant. Perez-Gomez (1982), Agrest (2000) and Vesely (2014) consider the value of theoretical projects in the exploration of the sublime. Prior to the Enlightenment, theoretical projects had no place, but following the work of Piranesi, Boullée and Ledoux, Architecture was engaged with explaining the "revelation of God through a better understanding of this works" (PerezGomez, 1982, p.5) and as explorations of the infinite, "where space escapes the boundaries of the perspectival box" (Agrest, 2000, p.165). Thus, the representation of imaginary environments, new ways of drawing and architectural exploration of subjective concepts surrounding the sublime create a foundation for an architectural theory to emerge as distinct from architectural practice.

An example of the negotiation between Enlightenment theory and architectural practice can be witnessed in the legacy of Durand. Madrazo (1994) explains how before this point, architecture had not kept in step with the developments in science; as a consequence, Durand was challenged with developing an education based on principles that bridged the gap between science and art. He taught a method at L'Ecole Polytechnique which distilled architecture into "a game of formal combinations facilitated by a grid...this was his mechanism of composition" (Perez-Gomez, 1982, p.5). Durand's theory developed as a systematic and logical process, beginning with a study of historic examples to identify common themes and drawing together an elemental pattern book (Perez-Gomez, 1982; Madrazo, 1994). This process culminated in a set of principles, drawing on typology and composition to direct the choice and arrangement of elements such as wall, columns and arches (Madrazo, 1994; Vesely, 2004). Through this revolutionary, systemised method of teaching, Durand addressed tensions between subjectivity and objectivity and art versus science, "Architecture is a science and an art all at the same time: like a science, architecture demands knowledge; like art, it requires talent" (Durand as quoted in Madrazo, 1994, p.20).

Vesely discussing the impact of Durand's legacy argues that discarding established symbolism to focus on instrumentality led to voids in significance and was the origin of the "contemporary crisis of meaning" (Vesely, 2004, p.242). Similarly, Perez-Gomez (1982) considers that Durand's systemised approach forced a choice between objective pragmatism and subjective poetry, steeped in meaning. Goudeau (2015) defends Durand 
against the retrospective criticism of his legacy, asserting that Durand's architecture demonstrates a hierarchy of form and that "visual strength" is apparent in his engravings. This argument is revealing; it demonstrates the point that Enlightenment ideas of autonomy, subjectivity and the sublime are most effectively realised through representation and theory, whereby the architect's autonomy in realising the built form may be subject to negotiation.

Lyotard (1985), Frampton (1992), Vidler (2002) and Vesely (2004) refer to the architectural philosophy of the Enlightenment and the work of Durand in particular, as the beginning of modernism. Certainly, in response to Enlightenment philosophy, the image of the architect evolves beyond the architect as polymath and artist and the image of the autonomous genius emerges, together with cultural notions of subjectivity and taste. Although there is accordance between the way the architecture developed during the Enlightenment and the contemporary discipline, there is a hiatus in the A-historical movement in architecture during the Industrial Revolution. Here, architectural expression follows divergent paths and the image of the architect is shaped by a context of exponential urban growth.

\subsection{THE INDUSTRIAL REVOLUTION}

The previous section describes how the Enlightenment galvanised an era of invention by embracing reflexivity and celebrating 'the idea'; bringing about the concept of human agency. The next significant development in the image of the architect began during the Industrial Revolution; a catalyst for enormous change first in the economic and social structure of Great Britain, then globally. Wallerstein (1974) and De Vries (1994) outline how the interconnectivity of economic and cultural networks, facilitated economic growth and, therefore, the spread of industrialization. Consequently, due to the colonial and mercantile character of late $18^{\text {th }}$ century Britain, ideas connected to progress and change spread quickly and widely. This section explores how the rapid and radical changes in British society during the Industrial Revolution influences the image of the architect. The discussion centres on the professionalisation of architects, exploring changes to client type and the importance of status.

In explaining the development of the image of an architect during this period, an examination of the extent and features of social change is germane to the discussion. Davies (1962), Wallerstein (1974) and Smith (2014), describe the movement of labour from agrarian to industrial economies, catalysing exponential urban growth and a demand 
for major infrastructure and civic building projects (Larson, 1977; Cuff, 1991; Deamer, 2013). An acute awareness since the French Revolution of social unrest and increased political activity amongst the masses (Froud, 2015), instigated a patriarchal society's response to managing social challenge in the form of philanthropic development. Consequently, social and economic change triggered urban construction projects through new demand from social infrastructure. Samuel (2018) identifies that “... socio- technical change -new health requirements, new materials, new structural possibilities... these required new forms of representation as well as new collective, and sometimes global, ways of working,” (Samuel, 2018, p.29). Crinson and Lubbock (1994) and Dodd (2015) explore the development of major building firms operating under new industrial scaledmodels. They describe a system of dominant contractors and developers whose construction practices emulated the work structure of a factory, creating a division of labour in the interest of efficiency and profit. This construction model undermined the traditional role of the master builder and turned craftsmen into subcontractors; it subsumed all involved in the building process, including engineers, surveyors and architects who "seemed to be becoming merely design specialists within the system" (Crinson \& Lubbock, 1994, p.43). Consequently, increasing complexity and new economic transactional models effected a re-alignment of workflow from capitalist clients or building firms rather than aristocratic or ecclesiastical patrons and the new client base effected a transformation in the architect's role (Larson, 1977; Cuff, 1991; Deamer, 2013).

From the outset, the structure of architectural training promoted elitism which reflected the hierarchies of society. Larson (1977), Cuff (1991), Stevens (1998) and Till (2009) recognise the influence of L' Ecole de Beaux-Arts model in the cultivation of architects. Cuff describes how "...Americans who ventured to Paris and the Ecole were struck by the atelier system" (Cuff, 1991, p.28) bringing the system to America poststudy. Cuff (1991), Stevens (1998), Till (2009) and Powers (2015) compare the BeauxArts' Atelier system to a layered class-based society; it was elitist in nature, concerned not just in developing academic skills but also "cultural capital" (Stevens, 1998, p.197). Powers (2015) describes how learning in the Atelier system was structured around teaching studios with a master, this created a cycle that generated not only developed learning but also reinforced and protected specific cultural norms, or the image, of the architect.

Although Great Britain did not possess an institutional model as ubiquitous as the European Academies, architectural training was not equitable. Exposure to intellectual 
learning was not based on talent; rather the ability to pay for a pupillage, poorer students working through an apprenticeship would be consigned to menial tasks (Powers, 2015). Crinson and Lubbock (1994) describe the increased prominence of pupillages throughout the eighteenth century as there was a financial incentive for architects to offer pupillage over apprenticeship. Within this system the image of the "gentleman architect" is inculcated. Crinson and Lubbock (1994) examine Soane's system of pupillage, where greater access was made available to ideas and resources, such as at the Royal Academy, helped shape the image of an architect:

Soane's ideal was of an architect who was a poetic designer, an intellectual and a manager imbued with high ethics, who could lead by virtue of his very distance from mechanical work (Crinson \& Lubbock, 1994 p.26).

This role emphasised an office-based approach to architecture, where drawings and specifications would be prepared and through which the builder would be instructed. The separation of the architect from craftsman implies a separation according to status, particularly given pupillage was available only to a wealthier section of society. The growth of pupillage and the increasing influence of architects who had no relationship to a crafts-based background, initiated a change in the balance of people entering the discipline towards those with a higher social status with the consequence of raising the overall status of architects. As Cuff observes, in this period "...only the aristocracy was able to go to college in America and abroad" (Cuff, 1991, p.24), therefore, to focus on intellectual learning, rather than the hands-on training of apprenticeships, encourages the image of architects as an elite and the separation of architect from craftsman. Thus, in the Industrial Revolution, the image of the architect as artist evolved to form the image of the gentleman architect.

\section{The Growth of Professional Institutions}

The protection of status was a key feature in the development of architecture as a profession in Great Britain. Larson (1977), Cuff (1991), Crinson and Lubbock (1994) and Powers (2015) describe how by the end of the eighteenth century the role could be undertaken by anyone, "if any carpenter, jobbing builder or surveyor could become an architect simply by calling himself one and putting up a brass plate then those who had endured an expensive pupillage were defenceless" (Crinson \& Lubbock, 1994 p.3). The 
development of architecture into a distinct profession with a protected title can, therefore, be understood as a response to people from outside of the accepted background of architectural training working as an architect (Powers, 2015). This point demonstrates the desire to define the role on the grounds of a particular form of expertise, within a particular section of society. Larson (1977), Blau (1984) and Ahuja et al. (2017) observe "historically, higher levels of prestige and autonomy have been bestowed on professionals than other occupational groups because of their claims to unique knowledge and skills" (Ahuja et al., 2017, p4). Larson (1977), Cuff (1991), Stevens (1998), Till (2009) and Powers (2015) all concur that the professional role of the architect is shaped by the aspirations of the upper-class entrants into the discipline and registration was the mechanism for protecting this status.

Consequently, professional status became a means of reassuring the public of the expertise on offer (Larson, 1977; Cuff, 1991 and Powers, 2015). Civil engineering preempted the rise of professions by convening the Institute of Civil Engineers in 1818, sixteen years ahead of the Institute of British Architects. In doing so, they claimed the objective aspects of the trade, "the arts and sciences of engineering" (Samuel, 2018, p.30). Thus, as architects were defining their specialism, “...the only element in architecture to which some other professional group did not have a prior or better claim, was 'art'" (Saint, 1983, p.61). Thus, through professionalisation, the image of the architect as an artist became encapsulated.

The founding of the RIBA in $1834^{2}$ was the fulcrum of the professionalisation of architecture. Its core purpose, as laid out in its 'prospectus for formation' as being for "the advancement of the knowledge of architects" (Samuel, 2018, p.30). The RIBA can, therefore, be seen to exemplify the aspirations for the image of an architect; Donaldson, its first secretary, describes it as a society of "men of taste, men of science, men of honour" (Donaldson as quoted in Froud, 2015, p.47), reinforcing Soane's gentlemanly model of the architect whereby the qualities of judgement and integrity are encouraged.

Although it was founded in London, the interconnectivity of Great Britain through economic networks and its status as a colonial nation, meant that the ideas connected to the professional image of an architect, reinforced through a professional body, was shaped through the international transaction. Cuff (1991), Crinson and

${ }^{2}$ The Institute of Architects was granted a Royal Charter in 1837 to become the Royal Institute of British Architects (RIBA) (Samuel, 2018). 
Lubbock (1994) and Stevens (1998) discuss how the development of the image of the professional architect occurs through transfer of ideas to and from the Beaux-Arts in France, the RIBA in Great Britain and the USA and Australasia ${ }^{3}$. The RIBA reinforced and disseminated values of a learned society through its structure, focusing the direction of architectural learning through its available resources, such as the RIBA library, lectures and reports (Till, 2009; Samuel, 2018). Once established, the RIBA's education system upheld the image of the architects as gentleman and artist, with associated qualities of taste and honour.

Hence, the RIBA encapsulated the image, the specialism and competence of the architect through its structure. Larson (1977), Stevens (1998), Till (2009) and Samuel (2018) identify the foundation of the RIBA as an act of self-interest, to control the market "by the professional's claim of possessing a superior knowledge, which should, therefore, be free of lay evaluation and protected from inexpert interference" (Larson as quoted in Till, 2009; p.17). However, it could be considered more an act of evolution. Contractors' firms threatened the architects' autonomy and other building professionals were becoming established, setting up their own professional structures and garnering areas of expertise which could as easily have fallen within the architects' scope. Consequently, the professional image of the architect was defined partly through the ideals of the time, but also through what was left behind by more enterprising professions.

The professional architect evolves through the Industrial Revolution from decisions made at a time of seismic shifts in society, as well as economic and cultural change. The architect's image is narrowed into distinct skills and traits that speak of hierarchy, status, taste and honour; the focus was on aesthetic and intellectual skills, eschewing the pragmatic and instrumental. The image of the professional had a wideranging and enduring impact but was enshrined according to values specified in the nineteenth century, thus, shaping the image of the gentleman architect.

\subsection{THE MODERN MOVEMENT}

The previous section 2.5 described how the image of the architect evolves in response to rapid growth and urban intensification, but also reflecting the social hierarchies of the Industrial Revolution. As with the Industrial Revolution, the image of

3 American Institute of Architects (AIA), 1857; New Zealand Institute of Architects (NZIA), 1905; Australian Institute of Architects (AIA), 1930. 
the architect is shaped during the modern movement by context and architects' response to it. Key catalysts for change in the first half of the twentieth century were the increasing scale of industrialisation leading to rapid urban development, but also economic and political shifts resulting from global conflict. Harvey identifies that "trauma of world war and its political and intellectual response...opened the way to a consideration of what modernity offers" (Harvey, 1990, p.30). The failure of the ruling classes, as evidenced through the war-torn nations, created the opportunity for an architectural movement which spoke of the emancipation of the people rather than historical structures of authority. Ghirardo (1996), Heynen (1999), Huxtable (2008) and Murphy (2016) describe the diversity in architectural theory during the modern period; there was no consensus about the essence of architecture. Nevertheless, Ghirardo observes, despite the range of architects' theory, “they retained as an underlying constant a belief in the power of form to transform the world" (Ghirardo, 1996, p.9). Consequently, as Eisenman (1984), Jencks (1973), Harvey (1990), Ghirardo (1996), Huxtable (2008) and Till (2009) describe, the modernist believes in the power of architecture to change society, thus invoking the image of the heroic architect.

The heroic architect comprises notions of genius and morality; Jencks (1973), Saint (1983), Harvey (1990) and Richards (2015) specifically describe the heroic image connected to modernist utopianism. Richards (2015) explores the meaning of heroic, observing that morality connects heroes but also good intention, judgement and action. Le Corbusier encapsulates this image when he writes in Vers une Architecture, "It is a question of building which is at the root of the social unrest of to-day; architecture or revolution" (Le Corbusier \& Etchells, 1986, p.169). McLeod (1983) also reveals Le Corbusier's radical challenge to the notions of family and home when she quotes "The house will no longer be an archaic entity.... then object of the devotion on which the cult of the family and the race has so long been centrated" (McCleod, 1983, p.140).

Accordingly, reform within the modern movement touches all aspects of human life from the macro of social reform through to the home and the everyday object.

In exploring the idea of modern, it is worth underlining the implication of temporality. Habermas refers to a belief in the "the infinite progress of knowledge" (Habermas, 1983/2005, p.99) whereas Heynen (1999) defines modern as new, but frames modernisation as a transient state, linked to upheaval and change, different from the past and evolving into the future. Modernism, however, is a cultural concept which embraces progress and rejects the past. Calinescu underlines this point, describing how: 
...an irreversible split occurred between modernity as a stage of Western civili[s]ation-a product of scientific and technological progress, of the industrial revolution, of the sweeping economic changes brought about by capitalism-and modernity as an aesthetic concept (Calinescu, 1987, p.41).

Harvey (1990), Heynen (1999), Vesely (2004) and Vidler (2008) identify modernism as a return to the underlying precepts of the Enlightenment, specifically, notions of autonomy and genius and the pursuit of a rational, A-historic approach, which rejects the traditional symbols of autocracy. The concept of a tabula rasa, or clean slate, emerges of contextless architecture, eschewing cultural traditions to enable society to start anew through an international language of modernism. The tabula rasa also reflects the Enlightenment philosophy of man's dominion over nature. Heynen (1999) and Huxtable (2008) remark on modernists' disregard for physical contexts such as the existing city and terrain, instead, focusing on values drawn from the machine age - cleanliness and machine-like architecture, which is rational and unadorned. Quoting Sullivan's often utilised phrase that 'form follows function', Eisenman (1984) explains how traditional forms of representation are rejected, and modern architectural expression is driven by the building's use. Thus, buildings are stripped of motifs and modernism demonstrates its autonomy through a self-referential language of pure form (Vidler, 2008). Considering the expression of pure form further, Till describes this process of architectural autonomy, whereby “...the modern project separated out the parts: nature/society, making/thinking, history/story, human/nonhuman." (Till, 2009, p.56). Following the idea of separation, Heynen describes modernism as a "rupture with tradition and has a profound impact on ways of life and daily habits." (Heynen, 1999, p.4). The implication here is one of imposition and disjunction, an analysis which is supported by Murphy (2016) who refers to the period as comprising poorly resolved experimentation. Comparably, Huxtable (2008) describes Le Corbusier's Voisin plan as devoid of humanity, “...wrong-headed schemes insensitive to the values of civili[s]ation and art" (Huxtable, 2008, p.159). Thus, an A-historic approach to living; from highly ordered civic space through to the machinemade dwelling, generated design based on an untested architectural theory for a new way of living rather than from an actual appreciation of how people think.

Through its autonomy, the theory embracing new technology and rejecting traditional meaning, fails to acknowledge the social and psychological impact on communities from such radical change. The effects of mass urban redevelopment on the 
modernist principles become apparent in the post-war period. Jencks (1973), McLeod (1983), Harvey (1990), Ghirardo (1996) and Deamer (2013) describe this late modern period as an exercise in pragmatism and cost-efficiency rather than a pursuit of pure modernist philosophy and became the architecture of the establishment rather than that of the avant-garde. Modernist rationalism "... became identified with the bureaucracies that commissioned, inhabited and sometimes even designed it” (Jencks, 1973, p.41), particularly mass house building, constructed along Taylorist principles of efficiency and density (McCleod 1983). Ghirardo identifies problems arising from a mimetic approach, when "designers followed the modern movement planning rather that their own instincts about urban neighbo[u]rhoods" (Ghirardo, 1996, p.13) thus, failing to engage with the possible implications of such a radical shift in the urban structure on society.

Although modernism was designed for the people, the people were not given a voice, it was an architecture which was not conceived to support individuals' rights to aesthetic expression. Similarly, the state-run mechanisms for managing public housing more resembled the autocracies they were designed to deny. Harvey (1990) underlines this point when recalling the ways public housing tenants were prohibited from changing their homes. Likewise, Bauman (1990) asserts that public housing projects meant "disempowerment of communal self-management...human conduct lost its appearance of naturalness" (Bauman, 1990, p.157). Consequently, rather than liberating the people, the rational order led to people feeling alienated in their communities. The architect, as creator of these failing urban models, was not perceived by the public as heroic, but arrogant and architectural autonomy became linked to notions of control; ultimately the architect's judgement is shown to be fallible. Ghirardo describes how the rational aesthetic was, “... a building style that came to be seen as boring, indifferent to the surroundings..." (Ghirardo, 1996, p.13) and Murphy, describing utopianism as “...downright dangerous experiments on an unsuspecting public, who in the fullness of time bravely rejected this arrogant paternalism" (Murphy, 2016). Moreover, the term "hero" architect is used by Cuff (1991); Stevens (1998); Till (2009) and Samuel (2018) with a hint of derision, indicating "...the distancing of the artist/architect from the sociocultural context of his or her work" (Groat, 1993, p.6).

Although the heroic persona forms a key part of the image of the architect, current texts indicate the harm caused to society but also to the public image of the architect from the concept. Changes to the architect's status occur following the modern period, as the protected functions of the architect come under scrutiny. Blau (1984), 
Gutman (1988), Symes et al. (1995) and Duffy and Hutton (1998) describe the erosion of the role; whilst Cohen et al. (2005), Carrio (2015) and Larson (2018) observe how the authority of the architect is questioned. Architecturally, the period between modernism and the contemporary setting has seen multifarious theoretical and practical explorations, as well as design movements to challenge the inhumane precepts of modernism (Harvey, 1990; Salingaros \& Mehaffy, 2006; Till, 2009). Despite these developments, the image of the architect has developed little, with the most recent manifestation, the "starchitect" (Ponzini, 2014), resembling the modernist heroic counterpart, perhaps muting the moral aspects of the identity (Schumacher, 1997). The rise of commercialism in the 1980s sees architectural production split between the everyday architect in practice working under increasingly competitive conditions, and the glamour of the star, whereby an architectural elite endures. The concept of the star architect is attached to corporate identity through iconic, signature buildings (Ellin, 1999; Jencks, 2006 and Sudjic, 2005), reinforcing the concept of genius but also the gap between a glamorous image and the actuality of the everyday architect working in practice.

\subsection{CONCLUDING DISCUSSION}

Through this chapter of the thesis, the image of the architect is shown to be cumulative, built from layers, which generated in response to the evolving economic, political and social contexts. The first surviving description of the architect, by Vitruvius, defines the image of the architect as a polymath but also reveals aspirations towards status. These two themes are consistent through the subsequent development of the image, which then changes to include the identities of artist, autonomous genius, gentleman and hero. The development of the image demonstrates that it is fluid and the architect is adaptive, however, K. Michael Hays, as cited in Till, observes that this adaptation occurs through external forces of change rather than directly engaging with the catalysts for change, architects undertake "an internalised redefinition of architecture in the face of them" (Till, 2009, p. 20). However, acknowledging that context is material to the formation of the image will facilitate discussions in the subsequent chapters about the endurance of traits and their relevance within the contemporary and evolving context. 


\section{CHAPTER 3}

\section{Tension, Challenges and Negotiation}

\subsection{INTRODUCTION}

The previous Chapter 2 traced the historical development of the image of the architect and explicated the way that the image has evolved as a layering of identities in response to changes in context. The study concluded with a professional identity, which is a polymath, creative genius and hero. Further, key characteristics embedded within these identities are autonomy (Johnson, 1994; Frampton, 1991; Heynen, 1999) and elitism (Stevens, 1998; Till, 2009). Chapter 2 concluded with the image of the hero evolving into the star architect. Ahuja et al. refer to the "multiple and conflicting identities" (Ahuja et al., 2017, p.3) which have led to the marginalisation of the architect (Hyde, 2012; Ahuja et al., 2017). Additionally, the status and role of the architect have changed in part due to societal changes whereby the infallibility of the specialist is questioned (Carrio, 2015; Nichols, 2017; Clarke \& Newman, 2017; Larson, 2018). The purpose of this chapter is to discuss the implications of this shift in the architect's role, on the architect's image. First, the gap between the image and the actuality of practice is analysed, to explicate tension within the architect's role. Second, the ways the emerging context creates challenges to the architect's role is explored. Third, opportunities for negotiating these challenges are discussed, observing how approaches to the discipline are reviewed and the prevailing image is being re-defined.

\subsection{TENSION}

Studies into the impact on architectural practice from changes in the construction industry and an evolving context have been undertaken from different perspectives. Blau (1984) and Gutman (1988) viewed the phenomenon from a sociological standpoint, whereas academics with architectural backgrounds explore the gap between the image of the architect and the actuality of practice from a position of familiarity (Cuff, 1991; Winch \& Schneider, 1993 and Symes, Eley \& Seidel, 1995). Within this academic framework, Blau (1984) and Cuff (1991) observe social structures within architectural education and practice to unpick the concept of the autonomous hero and reveal architecture as a social act. In contrast, more recent studies explore tensions within the gap by researching 
individuals' experiences of job satisfaction, value or disillusionment (Sang, Ison, Dainty \& Powell, 2009; Styhre \& Gluch, 2009; Caven \& Diop, 2012 and Ahuja et al., 2017). This section explores elements of the image which are subject to tension, specifically observing elitism and autonomy and their effect on the gap between the image and the actuality of practice.

\section{The Culture of an Elite}

Explorations of architectural culture identify how conventions within the profession resist adaptation. Tension instigated by changing context is thus magnified by a profession that has not sufficiently adapted its image in response. Garry Stevens' (1998) analysis draws on Bourdieu, specifically the concept of cultural capital, to dissect architectural culture through a critique of its elitist foundations. Stevens' exploration sits aside from other studies into architecture's sociology, as he focuses on the academic system to contend that entrants into the profession derive from a favoured social background. Stevens argues that architectural culture reinforces elitist values of 'culture' and 'taste', transmitted through academic institutions. Stevens (1998) is supported by Anthony (2001), Goldschmidt (2003) and Bachman \& Bachman (2009) who argue that academic institutions engage in "...the formal and explicit teaching of formal and explicit knowledge and skills...the only possible means of transferring embodied cultural capital" (Stevens, 1998, p.197). Stevens proposes that within this environment, those who are most likely to succeed are the people who are most conversant with the cultural norms of architectural society, rather than those with talent; this proposition is contradictory to the concept of genius. Stevens' theory follows a particularly focused line of argument which does not account for divergent social origins or, indeed, interrogate the wider motivations of entrants into the profession, however, it is illustrative of the mechanisms through which the image of the architect is perpetuated.

Till (2009) concurs with Stevens' historical tracings of the mechanisms through which the elitist value system is propagated, referring to the École des Beaux-Arts as a template for this, a subject which has been discussed in Chapter 2 (section 2.4). Till comments, "the walls of the black box protect architects from the contingencies of the world beyond, allowing them to develop theories and practices unfettered by others" (Till, 2009, pp.18-19). Unlike Stevens, however, Till disentangles the notion of autonomy, suggesting "it gives a sense of security but is ultimately fragile in the face of the rising tides of forces beyond" (Till, 2009, p.19) thus, contending that architecture must become 
contingent on the emerging context. The risks for an architectural culture which ignores the effects of a changing context is supported by Hill (2012) who warns:

From within, it is difficult to even perceive, and so to question, the deeper values, motives, models or possibilities for the profession; hence may professional bodies tend to be slowly fossilising within the compacting strata of their habits, discourse, and silent assumptions (Hill, 2012, p.7).

And so, through Stevens (1998), Till (2009) and Hill (2012), the argument is made that the image of the architect is reinforced by architectural institutions, but also that ideas attached to the image are becoming inapplicable within the emerging context.

Stevens and Till consider the role that pedagogy plays in perpetuating an anachronistic image. Larson (1977) observes that the investment attached to achieving professional training, both temporally and financially, is significant, therefore, patterns of behaviour and ways of speaking and thinking, or the culture, become ingrained. The scope of this thesis does not attempt to offer an in-depth analysis of the role pedagogy plays in reinforcing the image of the architect, however, this is a well-researched topic. Notably, Cuff (1991), Harriss and Froud (2015) and Samuel (2018) interrogate the gaps between pedagogy and practice and propose ways of building effective interrelationships between the two. Within these studies, the most ubiquitous conception confronted is the idea of the architect's autonomy.

\section{Autonomy}

The notion of autonomy is key to the image of the architect as genius, hero and star and, as discussed in the previous chapter, grew out of the enlightenment through the separation of disciplines into distinct silos and as a component of an individual's creativity. Autonomy has then been reinforced through the celebration of great architectural thinkers such as Le Corbusier, Frank Lloyd Wright and Rem Koolhaas (Miller, 1988; Saunders, 1997) and in popular representations as embodied, namely, through Ayn Rand's conception of Howard Roarke in the fountainhead (Saint, 1983; Cuff, 1991; Groat, 1993). But as Cuff observes:

... adjustment to reality was both a disappointment and a revelation. Disappointing because architecture can be so pure, so elegant, when a single architect creates without interference, and because the opportunities to do so are virtually non-existent (Cuff, 1991, p.4). 
Certainly, the revelation Cuff describes, that architecture is a social act rather than the product of a single autonomous genius, is a common theme amongst literature exploring the actuality of architectural practice (Schneider \& Till, 2009; Imrie \& Street, 2014 and Samuel, 2018). The first challenge to the notion of architects' autonomy is the role of a commissioning client; in most circumstances, the architect acts on behalf of another. Johnson cites Alan Colquhoun's proposal that “Architecture's expensiveness inevitably binds it to the sources of finance and power" (1994, p. 120), inevitably leading to its dependency on a client or patron. This point accords with the key theme explored in Chapter 2 whereby the image of the architect evolves following changes in socio-political power bases. Exploring this point specifically, Johnson (1994) considers the relationship between architect and patron to be one of compatible, although not necessarily harmonious interests. He illustrates this reciprocity by considering patrons who enabled Hero architects such as Frugés with Le Corbusier or Rebay and Frank Lloyd Wright. Further, Johnson remarks that there is a difference within the more contemporary manifestation of client and architect which is identified as transactional in nature, thus Asymmetrically balanced. This distinction, therefore, suggests that creative autonomy is dependent on an architect's relationship with their commissioning entity.

Although Johnson's analysis of the client versus patron relationship is illustrative of restrictions to architectural autonomy, Frampton (1991) expands the field of vision to observe that as architecture exists within a society, it is the least autonomous of the arts. Frampton argues that choices made, such as "typology (the institution), topography (the context), and tectonics (the mode of construction)" (Frampton, 1991, p.22) are influenced by extraneous factors. Frampton particularly identifies the paradox that seemingly self-referential, idiosyncratic architecture, is contingent on an interaction between architectural design and marketability. Frampton's comments on architecture's dependency are shared by Blau (1984), Cuff (1991), Stevens (1998), Jones (2009) and Till (2009). Correspondingly, Imrie and Street (2014) in writing about the socialisation of architects, remark that an ambition to work independently of social relationships is reinforced by a distinctive professional structure, reiterating the concept of elitist culture. Nevertheless, Imrie and Street observe, “...Architects, or any professional, are not disconnected from social contexts, and are co-constituted by their emplacement in networks..." (Imrie \& Street, 2014, p.726). Thus, autonomy is misplaced in a cultural enterprise such as architecture, which is dependent on social interaction. 


\subsection{CHALLENGE}

Following the exploration in section 3.2 of misapprehensions in the image of the architect, this section studies the effect of evolving context on the role of the architect. Gutman (1988), Winch \& Schneider, (1993), Symes et al. (1995) explore how sociopolitical shifts towards a globalised, neo-liberal model leads, not only to technological advances but also changes in the procurement methods. The changing context effects increased competition within the industry but also from new entrants into the field offering specific services. Eraut observes that "increasing financial power of developers and construction companies has redefined interprofessional relationships in larger projects" (Eraut, 2001, p.4), thus, the architect's role is being challenged. Eraut explains that delivery agents are the main commissioner of architects' services on larger construction projects and in these cases, architects provide services as part of a larger complex team, often managed by others. Thus, Eraut introduces the key change to architects' role within the contemporary context, the overarching strategic control has changed and architects contribute to a spectrum of services offered by a multi-faceted project team. Cohen et al., (2005) concur with Eraut that increasingly complex projects have effected a shift towards collaborative working; correspondingly, Dan Hill summarises, “... as the world became more complex, architecture's seat at the table was crowded out, as one voice among many" (Hill, 2012, p.10). Thus, within complex building projects, the architect's role has changed; the project team hierarchy has shifted and overarching strategic roles are often undertaken by others. Evidently, the emerging context is leading to a change in the architect's role; this section explores how these changes are manifest.

\section{Practice Structure}

Earlier studies into the changing nature of architectural practices such as Gutman (1988), Cuff $(1991,1992)$ and Symes et al. (1995) observed how practices evolve their structure in response to challenges from the emerging context. However, Cuff cautions that eventually, the movement towards larger multi-disciplinary firms and small specialised practices could resolve in "draining the middle of its traditional, small - to medium-sized, full-service practices.” (Cuff, 1992, p.204). In support of Cuff's predictions, Jamieson's (2011) more recent study for the RIBA's Building Future, reports that “....medium-sized design-led practices employing between 30-100 of staff, are increasingly under threat from the parts of the industry... able to provide their services more cheaply, 
comprehensively and frequently" (Jamieson, 2011, p.25). Jamieson clarifies that medium practices are too large to sustain small projects but too small to provide the range of skills, technological investment and fee efficiencies of large or composite practices. The discussion will return to propositions for future practice typology in section 3.4, however, the implications of this development should be noted. Blau (1984), Gutman (1988), Cuff (1991, 1992), Symes et al. (1995) and Jamieson (2011) speak of the value of the medium practice as "traditional" (Gutman, 1988, p.5), "all rounders" and "ideal” (Symes et al., 1995, p. 8-9). Blau clarifies that the medium-sized practice represents the best of both worlds; providing sufficient structure and resource to support significant projects, whilst maintaining “collective voice" (Blau, 1984, p.31), or influence in offices' decision making. Blau (1984) contends that within large offices, employees have less exposure to tasks and less influence over office proceedings; this reflects a rationalised system. To clarify, Blau tracks the origins of rationalisation to capitalist philosophy. It is a concept which relies on objective evidencing of value based on efficiency and is delivered through the division of labour which, she suggests, can lead to de-skilling. Notably, Blau observes “'Organisational rationality is found to undermine the scope of professionals' theoretical and operative knowledge..." (Blau, 1984, p.26). Consequently, rationalisation leads to a fragmentation of skills which questions the image of the architect as polymath.

\section{Fragmentation}

A central aspect of the image of the architect is the identity as a polymath; demonstrating skills, which cross multiple fields of knowledge. Traditionally, the architect was responsible for a design from its conception through to the end of construction; maintaining the design concept and quality throughout from an overarching, strategic position. Within this study, fragmentation refers to splitting this overarching role into different services, potentially undertaken by others; but also refers to the separation of design stages into discrete packages of work. Cuff observes “...most fundamental to architecture is the marriage of conception and execution, aesthetics and materiality" (Cuff, 1992, p.204), outlining the holistic nature of the architect's role. This statement introduces Cuff's exploration into the emergence of split-commissions whereby two architectural firms divide elements of the architectural delivery of larger or more prestigious projects. Cuff recognises the mutual benefits as, "a strategic way of accepting work that is too large for a firm” or, for the delivery firms, enables “...their people a chance to work on high-profile projects" (Cuff, 1992, p.208). However, it also introduces 
a question mark over the coherence of authorship and, more specifically, the holistic nature of the architect's role.

Additionally, Eraut (2001) observes that professionals' authority within this context and are, in fact, subordinate to the overarching manager. Consequently, the trend for seeking 'best value' through rationalised production practices can be seen to undermine not only the architect's role, but also its authority. This conflicting issue is documented by Ahuja et al. who observe:

...architectural services increasingly fragmented and the proliferation of specialist consultants... many architects noted that the aesthetic dimension of architectural work is under-valued (Ahuja et al., 2017, p. 12).

In contrast, Tombesi (2012) discusses fragmentation where complex building projects effect changing production models. He acknowledges challenges within the profession from separating the role as, "....architectural culture has always found it difficult reaching clarity on this, partly because of the objectively ambiguous nature of services" but also from "reconciling the intellectual nature of professional work with the economic aspects" (Tombesi, 2012, p.119). Tombesi does consider, however, that within the emergent delivery processes fragmentation is inevitable. He, therefore, offers that within a multiunit environment, architects could have value through their specialist skills and ability to coordinate across fields of knowledge. Thus, within this type of system, Tombesi predicts:

... the recasting of labor as discussed could affect the governance of the procurement process and the position of the architect in this process by rendering the ability to coordinate, define, and manage design interface more important than any other task. (Tombesi, 2012, p 131).

Although Tombesi's theory foresees opportunities within fragmented production models, he acknowledges compatibility concerns with architectural culture, specifically the misapprehension of autonomy. This position is also documented by Cohen et al. (2005) who identify the discontent experienced by architects frustrated that their expectations of a creative core are not realised within their role. Through their research, Cohen et al. reveal that architects understand that their role must fit within emerging production processes, however, "the creative dimension of the building process is being sidelinedand with that the power enjoyed by the architect is being eroded" (Cohen et al., 2005, p.785). Therefore, the emerging context is seen to challenge the image of the polymath, 
by eroding the architect's overarching role within a project, but also fragmenting design stages leading to diffuse authorship. The effect of this is a sense that the value of the architect's position is being undermined.

\section{Undermining and $V$ alue}

In view of Blau's (1984) summary of efficiency based on objective notions of value, it is worth recalling how architecture as a discipline became detached from the objective realms of science (chapter 2). Powers observes the challenge that a focus on art poses, "judgements based on the arbitrariness of personal taste have to be objectified, codified and presented as almost beyond dispute" (Powers, 2015, p.5). Within the context of determinable values, where architecture focuses on subjective concepts such as aesthetic judgement, its value is less clear to define. Consequently, the creative identities around which the image of the architect has been constructed are not considered essential, rather a product which can either be manipulated for market or dispensed with as part of a value judgement exercise. As an illustration of this position, Till (2009) observes that reinforcing the image of the architect as artist conveys an:

...enigmatic quality that raises its value on the external market. It also affects the internal economy of the profession, with the "star" architects underpaying their staff, but offering an osmotic relationship with artistry in return (Till, 2009, p.160).

Therefore, Till's argument undertakes two definitions of the term, in that the star architect is highly valued (financially) as a marketable product but also as a cultural figurehead (esteem). Correspondingly, in this relationship model the staff members are not valued under either definition of the word. In support of this point, Caven and Diop (2012) observe that poor financial rewards featured within their Anglo-French study of architects, however these were considered to be counter-balanced, “...in providing additional intrinsic rewards alongside the aspects of creativity and having esteem" (Caven \& Diop, 2012, p.521). Nevertheless, Till (2009) and Caven and Diop's (2012) observations on low pay, correspond with Hill (2012), who interrogates the issue further, tracking this system through to competitive fee deals meaning "Most architects are poorly paid or work long hours to impossibly tight margins" (Hill, 2012, p.8). Competitive tendering based on cost means that the architect is presented with the quandary between working to the fee budget and delivering an inadequately resolved design or developing the project beyond the fee allowance, resulting in a poor hourly rate or profit. Contrary 
to Caven \& Diop, Ahuja et al. comment "architects struggle to be valued for the work that they actually do" (Ahuja et al. p.12) thus, the competitive fee environment alienates architects from the intrinsic value within the role.

The previous chapter described how creativity and alignment with the fine arts is central to the image of an architect. Managed project structures, which prioritise objective deliverables, can be observed to undermine the integrity of design concepts, thus, challenging the architect's aesthetic dominion. Citing Porter, Winch \& Schneider (1993), refer to architects' value within the hierarchy of a project and how this has been undermined through the emergence of the project manager as a distinct role. Winch \& Schneider observe this role is often fulfilled not, by architects but by surveyors, whereby the architect's services often become "little more than a works package" (Winch \& Schneider, 1993, p.928). Similarly, Frampton (2012) observes the move towards distinct service roles rather than a holistic architectural role is market driven but also remarks that the architectural profession is partly responsible for its loss of authority by focusing on architecture as an aesthetic output rather than a process. Frampton's criticism suggests architects have rather more control over circumstances than is available, nevertheless, the situation can be seen as the origin of the fragmented production practices discussed in the previous section.

The crisis of value in the architect's role is manifest through the architects' changing influence. The core identities of creativity and polymath are challenged in terms of their relevance to contemporary practice and poor remuneration indicates that industry does not economically value architectural input. Thus, although Caven and Diop (2012) consider that the profession holds intrinsic self-value, this fact is also challenged by "...the number of people choosing to leave the profession due to poor job satisfaction" (Sang et al., 2009, p.312). This contradiction indicates that the innate rewards of being an architect do not compensate for challenges from external factors. These challenges encapsulate the tension between the image of the architect and the actuality of practice. The next section will explore how architects that stay in the profession negotiate this tension and how their practices evolve in response. 


\subsection{NEGOTIATION}

Following on from the exploration within sections 3.2 of tension in the image of the architect and 3.3 of challenges to the architect's role, this section studies strategies for negotiating these issues. Winch \& Schneider (1993), Jamieson (2011), Salomon (2012) and Hyde (2012) propose ways of adapting architectural practices in response to the working context. Schneider \& Till (2009), Imrie and Street (2014) and Samuel (2018) make proposals for redefining the image, specifically notions of autonomy and elitism, by treating architecture as a social process. Within the difference between the literature described here, sits a common theme; all describe alternative models of working which provide new foci within the architects' role. The spaces between these texts are also populated with studies and theories, some of which will be touched on in the following analysis. The key purpose of this section is to create an overview of the existing literature which discusses the way architects are evolving how they work, with a focus on adapting practice to negotiate the role and negotiating the image.

\section{Adapting Practice}

The Royal Institute of British Architects (RIBA) issued research in 2017 titled Practice Resilience: How Architects Survive and Thrive During Challenging Times. Based on interviews with partners or directors of 21 practices in the UK, the report concluded that architecture is an industry of "creative problem solvers used to responding to change" (RIBA, 2017, p.1). It specifically identified four criteria, which facilitate practice resilience in the face of challenges from a changing context. These criteria are "Business-focused; innovative; adaptable; diverse and inclusive” (RIBA, 2017, p.1). This section will touch on all four of these themes, but with a specific focus on innovation and adaptation.

Winch \& Scheider (1993) commence their study into the strategic management of knowledge-based organisations by distinguishing such businesses as “...they have only the expertise of their staff as assets with which to trade" (Winch \& Schneider, 1993, p.923). Further elaborating on the nature of architectural practices, Winch \& Scheider categorise these as creative:

They are hired by clients to provide novel solutions to spatial problems, a process with inherently high levels of task uncertainty. To cope with these demands, their training emphasi[s]es innovation and problem solving, and this creative process is at the root of why many joined the profession. (Winch \& Scheider, 1993, p. 927). 
Certainly, a common theme within studies of architects working in practice (Cuff, 1992; Sang et al., 2009; Styhre \& Gluch, 2009; Caven \& Diop, 2012; Sturges, 2013; Ahuja et al., 2017 ) is that creative design shapes architects' decision to enter the profession.

Consequently, Winch \& Schneider (1993) position the architect's creative identity within the changing context and the need to win work. Drawing on various frameworks for demonstrating competence for creative practices working within a competitive market, Winch \& Schneider identify four strategies around which practices might coalesce. First is strong delivery or efficiency and reliability in delivering a product. This model describes practices undertaking simpler projects for a lower fee, but " ... at a higher level of profitability through effective organi[s]ation of the design process" (Winch \& Schneider, 1993, p.930). Second is strong experience, or an ability to manage complex processes such as challenging planning processes or negotiating multifaceted client structures. This model suggests either a multi-disciplinary practice or one with a reputation for delivering a specific project typology. Third, is a practice with strong ideas and is categorised by practices with “... a strong, even charismatic figurehead” (Winch \& Schneider, 1993, p.930) with a focus on innovation and creativity. Fourth is strong ambition which describes new practices with low experience but highly motivated to become a strong ideas practice. This model is described by Winch \& Schneider as "charging below average fees due to lack of reputation...this strategy is not sustainable in the longer term" (Winch \& Schneider, 1993, p.931). The long-term success of this strategy requires a competition win or the support of a patron. Winch \& Schneider's (1993) strategic framework provides a level of flexibility for practices to move between models, a consideration relying on is adaptability and an attitude to specialism or diversifying working practice. Specialising enables expertise to be developed along with a reputation within a particular field of work. It also facilitates smaller practices to operate within more complex project structures. Winch \& Schneider (1993), however, identify that with specialising, “...there are attendant risks...that the market may change" (Winch \& Schneider, 1993, p.933). They consequently propose that a strategic approach to practice must facilitate flexibility to expand into more diverse areas in response to changing context.

A more recent perspective on practice structure is outlined in a second RIBA publication titled The Future for Architects (Jamieson, 2011). The research is the result of a year-long study interviewing forty people from connected professions such as clients, architects, engineers, contractors and developers and looks ahead to the year 2025 to anticipate how architectural practice may evolve. The basis of the study is that the 
profession is considered insular, insufficiently engaged with a client's needs. Jamieson (2011) assessed that certain parts of the industry which focus on delivery would remain stable and emerging typologies would join them whereas other, more traditional, types of design practice are vulnerable. The types of structure which are predicted to thrive as the context continues to change can be categorised as either diverse models of practice or focused models.

Within diverse working practices, scale and sometimes, international reach, can be observed to offer resilience as the focus is on providing a spectrum of services but also the ability to streamline services to offer cost efficiency. This model accords with Winch \& Schneider's (1993) strong delivery strategy and is a response to an increasingly competitive fee environment as scale and diversity provide a level of risk protection. Also, in this category is traditional regional delivery practices, which “...provide a strong, no nonsense, local service" (Jamieson, 2011, p.20), based on an established reputation for service rather than design ambition. Additionally, Jamieson (2011) identifies the growth of practices based in emerging economies and these can also be considered to sit within Winch \& Schneider's (1993) strong delivery model. Tombesi (2001) also discusses the development of paradigms where architectural packages are outsourced within a globalised market, facilitated by digital communication networks, to draw on resources based in lower wage economies.

In terms of focused types of practice, Jamieson (2011) describes architects providing specialist niche services such as technical consultancy work; also, within this category are architects working in-house for developers or even subcontractors on specific technical works packages. These models of working can be seen to align with Winch \& Schneider's (1993) strong experience strategy and as a response to changes in procurement processes towards contractor or developer-led design and build contracts. Focused models are a pragmatic response to the changing context; however, as discussed earlier in this section, they are exposed to market volatility. As with the emerging economies paradigm, focused models also correspond with the concept of fragmentation (Blau, 1984; Tombesi, 2012; Ahuja et al., 2017) whereby a narrow focus can lead to deskilling (Blau, 1984).

The third development in practice type, as described by Jamieson (2011), dissolves roles entirely, working across a range of sectors, harnessing 'design thinking' to follow a diffuse market for services. This model is inherently flexible and challenges the image of the architect as a distinct role; it can also be considered to accord with Winch \& 
Schneider's (1993) prediction of how, in the face of a changing market, a practice might diversify. Finally, Jamieson's (2011) research identifies that practices are moving away from Winch \& Schneider's strong ambition model whereby small, boutique design studios are predicted to become less prevalent. As discussed in section 3.3, the study concurs with Gutman (1988) and Cuff (1992), that medium-sized design practices are vulnerable. Jamieson's (2011) findings, therefore, describe an industry which is adapting to the challenges from rationalisation, flowing from a capitalist economic model. Further, the predictions describe a two-tiered system with internationally renowned star architects maintaining significance, in line with Winch \& Schneider's (1993) description of a practice model based on strong ideas, and the remaining practice types illustrating a strong delivery or strong experience model. The picture described by Jamieson (2011) can, therefore, be considered to represent an increasingly challenging environment for architects wishing to exercise autonomy by working within smaller design practices or, indeed, setting up a new practice focused on creative innovation. The question remains, therefore, how do architects who are not (or do not wish to be) attached to larger offices or internationally recognised design studios, create opportunities for working innovatively through selfdirected creative practice?

Architectural theoretician David Salomon (2012) further explores the development of architectural practice and proposes a differing view, where he anticipates practices will follow three distinct categories. First are practices that are structured around interdisciplinary collaboration, treating architecture as a social process. This proposition accords with Winch \& Scheider's (1993) strong delivery or strong experience strategies and is consistent with Jamieson's (2011) description of global interdisciplinary consultancies, however, Salomon's collaborative model also encompasses theoretical work surrounding agency and relationality (Schneider \&Till, 2009; Imrie \& Street, 2014 and Samuel, 2018). Second is digital design specialists focused on computer-based networks. This proposition accords with Winch \& Scheider's (1993) strong experience strategy and could be linked to Jamieson's (2011) description of globalised practices such as inter-disciplinary or based in the emerging economies. However, Salomon's (2012) digital-design specialist also corresponds with Tombesi (2006) and Wiscome's (2006) work which outlines re-defined modes of working simultaneously across disciplines and facilitated by computer technology. Tombesi (2001) discusses how " design conception, production of working drawings, and site administration for the same project are carried out by components of the same organization located in different parts of the world" 
(Tombesi, 2001, p.171). Whereas, Wiscome (2006) advocates for a 'Co-evolutionary' design process across a range of fields such as on complex structures or ecological design. In Wiscome's model, design work is generated simultaneously across disciplines. Wiscome remarks, "Architects in this world might be forced to give up their afflictions of, heroism and genius, in favor of a new role as instigators of innovation" (Wiscome, 2006, p.65). Whereby the design process becomes an interchange between expertise, Wiscome sees this as an opportunity to evolve and innovate. The third category Salomon outlines are community-based design and build practices which recognise architecture's role "as an instrument for progressive social change" (Salomon, 2012, p.435). The difference, therefore, in Salomon's perspective from Jamieson (2011) is the perceived opportunity for architects' involvement at the conception of a project, through social enterprise. This means involvement at the earliest stages of the strategic design, or it may even involve speculatively generating projects. Illustrating Salomon's argument, Rory Hyde (2012) also writes about new forms of architectural practice drawing on existing examples of creative methods of working within the built community.

Hyde (2012) discusses the architects' 'crisis of relevance' at a time when society needs architectural interventions and solutions to problems. Rather than explore radical design solutions, he explores radical practice models which offer divergent responses to negotiate the challenges. In looking for the common denominator to link the practices, Hyde cites Cedric Price who famously remarked "the best solution to an architectural problem may not necessarily be a building" (Price as cited in Hyde, 2012, p.23). Through conversations with 17 people, Hyde's analysis draws on the work of architects, but also others from interconnected fields such as artists, urban policy makers, social activists, educators and theoreticians, to explore diverse responses to contemporary challenges. Within this diversity, there is a correlation with the three categories identified by Salomon but the most striking theme running through Hyde's studies is an element of entrepreneurialism. Hyde (2012) discusses Marcus Westbury's work at Renew Newcastle as a template for how architects could start to innovate using non-architectural strategies for generating architectural outcomes; it is a proposition that architects can harness strategic thinking and business development practices to create projects. In this case Hyde examines the initiative with social renewal at its heart, therefore, reinvigorating the architect's moral identity, however, the core focus is in strategically identifying a cultural gap within a local context and creating opportunities to resolve it Hyde (2012). 
The level of personal investment and vision required in the enterprise is outlined by Marcus Westbury in his conversation with Rory Hyde, “...I just got a chance to generate what I thought would work.... a lot of good people donated their time and energy..." (Westbury quoted in Hyde, 2012, p.175). The example, therefore, demonstrates skills in negotiating across a range of people strategically, politically and economically. It is a mechanism for change which requires creative thought, coordination skills and entrepreneurialism. This example sits amongst a collection of case studies which demonstrate how, redefining the image and the role of the architect can generate new opportunities by evolving strategies for working in practice.

\section{Negotiating the image}

Following the previous exploration into alternative routes for practice development and the earlier discussion on tension (section 3.2), the image of the architect is, within the contemporary context, a matter of negotiation. Challenging the culture of an architectural elite, Groat (1993) considers that to remain germane, architecture must engage across a wide cultural base and different disciplines. Cuff (2012) draws attention to the paradox of upholding the prevailing image as the image is anachronistic and prevents architects from effectively negotiating challenges from the emerging context. She resolves that "a shared model for architecture is as essential to the production of difference and innovation as the invention itself' (Cuff, 2012, p.391-2). Salomon (2012) on the other hand concludes that architects are not just negotiating beyond the image of the elite designer, but also beyond the image of the social activist. Further, he recognises that architectural practice is fragmented, with no single solution to the challenges experienced from the emerging context. These analyses agree, however, that practices are evolving and to negotiate the tension initiated by changing contexts, architects must refocus the image.

Section 3.2 identifies that the elitist culture and the autonomous identities restrict the architects' ability to negotiate the gap between the image and actuality of practice. Nicol and Pilling write about the public perception of architects, identifying the public's demand "...for architects to demonstrate greater sensitivity in their design responses to the built environment" (Nicol \& Pilling, 2000, p.3). Greater clarity in communicating the intention behind architects' proposals is also related, proposing that interpersonal relationships and transparency become key aspects of the architect's identity (Nicol \& Pilling, 2000). Flora Samuel (2018) also explores the public perception of architects and, 
writing more recently, recognises that architects' opaque role continues to be a concern. Samuel (2018) proposes that the lack of clarity is linked to the crisis of value in the profession, observing that architectural culture contributes to this further (Saint, 1983; Groat, 1993; Stevens, 1998 and Till, 2009). Samuel (2018) also identifies that the way architects speak, clothes they wear, and lack of diversity is characterised through media depictions, which reinforce the image of the architect (Powell \& Prasad, 2010; Stead \& Richards, 2014; Smitheram, Kidd \& Lam, 2018). The public image, together with the reputational damage of modernism and the prominence of the star architects lead Samuel (2018) to conclude that to tackle misapprehension "...the profession needs to be clear about what it knows and the value of what it knows" (Samuel, 2018, p. iii). Samuel's solution is for architects to provide evidence of their value, by re-framing architectural knowledge through practice-based research and then communicating this outwardly (Samuel, 2018; Hay, Samuel, Watson \& Bradbury, 2018).

Thus, the research outlined above indicates the need to re-frame the image of the architect and the way the image is communicated. Peggy Deamer (2015) also scrutinises the implications of the elitist culture of architects but, from an internal perspective. Deamer identifies that, as a profession “....we don't believe we do work... as a profession architecture produces designs, neither mere products nor services" (Deamer, 2015, p.61). In an earlier, but related, critique Deamer (2012) also interrogates architecture's failure to adopt a business-like approach, culminating in poor remuneration and long hours. The professional culture, therefore, based on the image of the gentleman architect, disconnected with the vulgarity of commerce (Stead \& Richards, 2014; Bernstein, 2015), ironically leads to the role being undervalued in the contemporary context. This observation feeds back into the discussions undertaken in the concluding remarks of section 3.3 of this chapter, whereby the innate rewards of being an architect do not compensate for the lack of perceived worth from external factors. Examining emancipation in architecture, Deamer (2012) predicts this tension in the image of the architect could be re-negotiated by a new generation of architects, “ $\ldots$ with their entrepreneurial expertise in open systems of exchange... uninterested in the obsolete limits that the profession of old exerted" (Deamer, 2012, p.87). Deamer, therefore, advocates for emerging architects with distinct skills to re-negotiate their value both within the hierarchy of the architecture studios but also the wider building industry.

The existing framework of research, therefore, supports the view that to negotiate tension in the image of the architect, the culture surrounding architects as an elite should 
be re-defined. To further illustrate this point, Tombesi (2012) suggests that the concept of architecture as a profession is becoming immaterial to its value and Deamer identifies that the distinction between disciplines is becoming more fluid, "designer is no longer equated with architect; fabricators, engineers, and software programmers can lay equal claim to authorial designation... control of the critical path is mingled with control of form" (Deamer, 2012, p.19). This fluidity also brings into focus the significance of autonomy within the contemporary image of the architect. Corresponding to the notion that collaborative working challenges the autonomous image, Till (2009) rejects the notion of a separate mode of practice and instead considers architecture to operate within a social network, referring to a "hybrid use of knowledge" (Till, 2009, p166). Imrie and Street (2014) also consider autonomy, first distinguishing the idea of architectural autonomy as self-referential, based on "ethical distancing of architects from the objects/subjects of their practices" (Imrie \& Street, 2014, p.724) and the concept of relational autonomy. Imrie and Street, then, describe a redefinition of the concept of autonomy as situating architects and their work within a social system. Specifically challenging the image of the Hero Architect, or "lone genius" (Till, 2009, p.151). Imrie and Street propose an architecture which is the product of discourse, "... autonomy is part of a social process in which to exercise agency, and self-determination, depends on the recognition of one's embeddedness into collective social formations" (Imrie \& Street, 2014, p.726). Hill (2003) also delineates professional knowledge as existing across social networks and being constructed around the understanding that design is never complete; the actions and interaction of others occurs as a discrete part of the process. Following a similar theory, Franck and Lepori (2007) advocate for an architecture which is designed with people's “...desires, sensory experiences, and everyday actions, rather than imposing preconceived ideas" (Franck \& Lepori, 2007, p.7), through which design becomes meaningful.

The concept of spatial agency (Kapp, Baltazar \& Morado, 2008, Doucet \& Cupers, 2009; Scheider and Till, 2009; Jenkins \& Forsyth, 2010 and Lorne, 2016) attracts much theoretical discussion. Doucet \& Cupers (2009), outline the broadness of the concept within a diffuse framework of interpretation; within the context of this discussion Schneider and Till's (2009) is taken: 
context in order to engage better with them in a transformative and emancipatory manner (Schneider \& Till, 2009, p.98).

Thus, having demonstrated in section 3.2 how tension arises in the autonomous image of the architect, arguing that professional knowledge is contingent on the experiences of others (Till, 2009), or that human activity has an architectural affect (Doucet \& Cupers, 2009) identifies how this aspect of the architect's image might be negotiated.

This section has outlined ways in which architects and theoreticians identify areas of tension and are challenging their image to re-negotiate the role in the changing context. Architects do this by harnessing creative and interpretative skills to bolster a responsive and adaptive nature. Emphasising this aspect of the architect's image provides an invaluable foundation for anticipating and adapting to further change.

\subsection{CONCLUDING DISCUSSION}

Through this chapter of the thesis, tension in the image of the architect is shown to have emerged from changes in the socio-economic context, which in turn led to change in the construction industry. These changes commenced around the time when architects' judgement and dependability were being scrutinised, leading to the profession's status and role being challenged. The literature review undertaken in chapters 2 and 3 identifies how architectural culture, which reinforces the image of an architectural elite and maintains ideals surrounding autonomy, does not reflect the actuality of architectural practice. Consequently, as the context evolves, the gap between the image of the architect and the actuality of architectural practice is increasing. The final section of this chapter reviewed existing literature discussing how architects evolve their work, by focusing on adaptive practice to re-negotiate the architect's role and how they are challenging the image. The review identifies a lack of research into architects' experiences of negotiating the challenges through viable, adaptive practice models, and how this affects the way the architect's role is comprehended. Chapter 4, which follows, builds on the theoretical research undertaken in this chapter, by discussing the effect of the image with architects in practice and their direct experience of tension and negotiation, focusing on the impact these issues have on the direction of their practice. 


\section{CHAPTER 4}

\section{Experiences from Within Practice}

\subsection{INTRODUCTION}

The previous chapters of this thesis have tracked how the image of the architect has been constructed and the emergence of a gap between this image and the actuality of architectural practice. Chapters 2 and 3 carried out both a historical study of the image and a theoretical review of the tensions and challenges faced by architects working in practice, recognising that there is a need to readdress how the architect's role is comprehended. Building on the existing literature, Chapter 4 presents an empirical investigation into these themes, gathering the direct experiences of eight architects working in practice in both New Zealand and the United Kingdom. As has been outlined in the methodology section of Chapter 1 , the investigation takes place through a series of semi-structured interviews which investigate the increasing gap between the image of the architect and the actuality of architectural practice. This chapter will also use the research data to understand how architects work subtly using their wide-ranging skills to achieve compromise, strategic thinking and creative judgement in intricate and thoughtful ways, to negotiate the gap between the image and actuality of architectural practice.

This chapter is divided into five sections, commencing with this introductory section 4.1, then unfolds as follows: Section 4.2 evaluates architects' experiences of image, drawing a distinction between the public image and how architects see themselves.

Section 4.3 describes architects' experiences of integral aspects of the role that do not form part of the image and are sources of frustration and tension. Section 4.4 discusses ways architects negotiate challenges in their work by emphasising the aspects of the role that they consider important. The final section, 4.5 is the concluding discussion, which identifies that the image upholds architecture as an aesthetic product rather than a process but that the process determines successful design. This section evaluates the themes emerging from the empirical study to conclude that the contemporary architect is a generalist, offering a range of highly developed skills, which should be capitalised on to enable architects to adapt deftly and negotiate the gap between the image and actuality of architectural practice. 


\subsection{THE EXPERIENCE OF IMAGE}

The foundational position of this thesis is that the image of the architect is at odds with the actuality of practice. Chapters 2 and 3 mapped out a history of how the prevailing image of the architect has been constructed from existing literature, concluding that identities of the polymath, artist, creative genius, gentleman and hero are linked to notions of knowledge, creativity, judgement, autonomy, elitism and morality. Sang et al. (2009) observe that misapprehension within the profession is due to "the anticipatory socialisation process for architects" which “...fostered inaccurate expectations of the profession in terms of scope for design and creativity" (Sang et al., 2009, p.317). Sang et al. also observe this misapprehension to be inversely related to direct exposure to the role before entering the profession, in other words those who knew an architect, had more grounded expectations than those who assumed "societal stereotypes" (Sang et al., 2009, p.319). Following this argument, the image of the architect is significant in explicating expectations of the role before working in practice.

\section{Effects of exposure on the image}

This section focuses on the image of the architect, but as viewed by the interview subjects. Architects were asked to describe what they thought the image of the architect was before becoming an architect. Their responses helped to understand if expectations are formed by stereotype, (image), or direct knowledge. Four of the eight architects had a connection to architecture before study through someone they knew; this gave them a clear understanding of what the role might entail:

My father was a draftsman in an architectural office...I think I imagined it would be a little bit different to the type of practice that he had (Architect\#1NZM4).

... because with my dad being an architect, I wanted to be an architect... by the time I became a teenager, I thought the stuff he did seemed quite dry...(Architect\#2UKM).

My father was in construction, he was an estimator so I was around construction a lot but there were no architects involved, it was all engineering (Architect\#3NZF).

...my granddad had been an architect, but it felt very distant because he was retired by then (Architect\#6UKF).

\footnotetext{
${ }^{4}$ Architect reference whereby \#1 is subject no. NZ / UK refers to country architect works in and $\mathrm{M} / \mathrm{F}=$ male or female. Refer to matrix (appendix vi) for further details.
} 
Two of these architects also had worked in an architects' office prior to studying:

I worked for an architect before I went to [university] and he was a one-man band in an office doing alteration work, so I got to see that side of it (Architect\#3NZF).

I went straight into practice as soon as I left school, I don't think I ever had that idea of the architect, it was given to me on a plate when I was 18...(Architect\#6UKF).

Prior knowledge meant these four respondents made an informed decision when embarking on a career in architecture, although the first two indicate that they believed their experience would be different from their relatives' precedent. In contrast, the two who also worked in architects' offices before studying considered their expectations of the role were well grounded.

The remaining four respondents had no direct access to the profession, although two of the interviewees had an oblique introduction through either building work on their parents' house or buildings in their local community:

I didn't have an image of what that was going to be apart from the historical references of Charles Rennie Mackintosh and the guy that did our extension at home (Architect\#8UKM).

...mum and dad always used to...show me buildings and places and exhibitions about architecture and I got really interested in the artistic practice of it (Architect\#7UKM).

Whereas the remaining two respondents had no clear recollection of how they were introduced to the role; their decision to study architecture came from a belief that it would be compatible with their interests, specifically creative skills:

I had partly thought of doing something more engineering based and then tried the artbased thing and I thought, well, maybe the middle ground is architecture (Architect\#4NZM).

I was one of these people when they were a kid, decided they were going to be an architect without knowing what it meant, particularly, but I think I was drawn to the idea of design discovery ... and testing stuff through drawing...(Architect\#5NZM).

Thus, the respondents report varying degrees of exposure to the role before entering higher education and this affected how they understood the actualities of being an architect. 


\section{Recalling the unschooled image.}

Next, the architects were asked to outline their understanding of the image of the architect prior to entering higher education. Architect\#7UKM was the only respondent to mention architecture's identity as a profession:

....as someone that chose to be an architect from my school days, I think it was not marketed artistically. It was marketed as a profession to do rather like law or medicine.

This statement is interesting, firstly because this initial perception of architecture as a profession was not reflected as central to the other respondents' understanding, but also that it was not why Architect\#7UKM chose to study architecture; aspects relating to the creative identity had drawn him into the profession.

...what interested me in architecture from a very early age is the capacity to influence things by drawing and design.

This concurs with the majority of the interviewees who recalled they were drawn to architecture because it is seen as creative. Architect\#8UKM was also drawn to the artistic side of the profession but also idealism, aligning with the heroic identity:

I thought I could make a difference as an architect. I see that's partly why I joined, I was good at drawing, problem solving.

Referring to prior knowledge, those who knew an architect before beginning their studies had a more sophisticated understanding of the role. Architect\#1NZM, whose father worked as a draftsman, expressed his interest in the intellectual development of the role, thus, drawing on the image of the polymath:

I like learning about things and understanding how things work...I always thought that architecture had that sense of knowledge and discovery around it...

Architect\#3NZF also had prior knowledge both through her father's work as an estimator but also through work experience. She initially studied interior design before moving into architecture, due to a desire for greater control over the entirety of a project. This draws on the autonomous image.

...[in] interiors, you can do some things autonomously but really the architect is the person that has control and I wanted to have that role... 
Therefore, when recalling their perceptions from the beginning of their careers, the respondents' comments assemble to form a skeletal image of the architect, encompassing themes of knowledge, creativity, autonomy and idealism which draw from the identities of the polymath, creative genius and hero; also mentioned is the image of the professional.

The study reveals that the idea architecture is a creative art is a common theme amongst the respondents. Most of them recalled creativity and discovery through drawing was integral to their choice to become an architect. However, the comments also reveal that viewed from outside of the profession the subjects held divergent notions of the role and comprehension grows with exposure to the profession. This analysis is further supported by respondents' observations that the full extent of what architects do and the complexity of the design process is not fully appreciated by those observing from the outside:

I think all of it is underpinned by a misunderstanding of what architects do, because I think most people don't really understand what it takes to design a building...(Architect\#2UKM).

...when I was studying and I would talk to some friends and I'd say 'yeah I'm studying architecture' and they'd say, 'So what exactly do architects do? ... you'd have to describe it and then they still wouldn't really get it...(Architect\#4NZM)

This suggests that architects do not adequately communicate the role outside the profession. This is similar to Samuel, who argues, "lack of clarity about what it is that architects know makes it very difficult for them to defend their territory" (Samuel, 2018, p. 6). The respondents were also asked to comment on the image of the architect from their position as experienced professionals. These discussions revealed a greater clarity and cohesion between the subjects' comments, suggesting there is a disparity between the public image of the architect and how architects see themselves. The following two sections (4.2.1 and 4.2.2) will analyse the disparity, commencing with how the interviewees comprehend the gap between the image and the actuality of the architect.

\subsubsection{Experiences of disparity in the image}

Most of the architects agreed that there is a difference between the public image of the architect, and how architects see themselves: 
When you talk to people outside the industry who see it as very prestigious and say, 'that's exciting, that's interesting' and there's the one within the industry which is very different (Architect\#6UKF).

Consequently, the architects were asked to describe what they thought the image was and what they think it should be. Elements of the image communicated by the subjects, is that the architect is seen to be privileged (Architect\#3NZF); ego driven (Architect\#6UKF); creative (Architect\#2UKM) and in control (Architect\#3NZF), demonstrating how elements of the image relating to the elite, creative genius and hero, uncovered in chapter 2 , resonate. However, there are additional implications attached to these characteristics which reinforce the image of the architect; first is a sense that the architect is aloof, separate from society, reinforcing the notion of elitism.

\section{Elitism}

As chapter 2 discusses, “...professions had their origins in the power base that both feudal lords and organizations linked to the Church had over their subjects and followers" (Carrio, 2015, p.172). Larson (1977), Stevens (1998) and Till (2009) propose that the act of encapsulating the discipline as a profession brought with it the concepts of exclusivity and status. The potency of architectural elitism is widely researched, however, the subjects' comments relating to their own work suggest there is a disparity between the external perception of an elite and their experience of practice. Before studying architecture, Architect\#4NZM had no direct experience of architecture, or personally knew people working in the profession. He describes how a local architect was talked about with interest because he lived differently to other people in the area:

I always thought the architect was something else that we, as the ordinary people, wouldn't have anything to do with.

The recollection of this local architect being somewhat of a local celebrity was built around his distinction from the people living nearby. Steven's refers to cultural capital as knowing, "all the subtle signs of cultivation-accent, manners, deportment, bearing, dress, attitudes, tastes, dispositions-cannot be obtained second-hand" (Stevens, 1998, p.196). Interestingly, most of the respondents did not consider themselves to be part of an elite, their motivation was in producing architecture not, necessarily in being an architect.

I've never found the profession exciting, I've found building exciting (Architect\#7UKM). 
Some respondents recognise the significance of cultural capital, but treat it more as a means of self-parody, than a means for reinforcing a privileged status:

...the parody of the black roll neck thing hadn't really hit me as an 18 year old! (Architect\#8UKM).

We're so full of these rules aren't we? We quite like it don't we in our little world?...So I mainly wear black and go into meetings and flourish my pen! (Architect\#6UKF).

Others observed that some architects maintain the concept of an architectural elite, but they did not consider this to be material to their role:

I think architects know what being an architect is like, but there are some architects who have this more glamorous image, but I think most architects don't (Architect\#3NZF).

Part of my strategy is to just avoid the crowd as much as I can, I'm not part of the London set ... maybe I'm becoming a bit boring, I just enjoy doing the work (Architect\#2UKM).

There is, thus, a difference between the perception of elitism within architecture and the experiences of the respondents. It is important to note that the data presented here is the record of respondents' self-reflection; the methodology of this research does not involve an ethnographic approach which would enable a more in-depth and detached assessment of this issue. The extent to which the architects engage with and reinforce the cultural norms of the architectural elite has not, therefore, been explored through observation as a part of this study. Nevertheless, the interviews reveal an awareness of the elite, and that this is reinforced within the profession, but being part of an elite is not considered material by any of the respondents.

\section{Creativity}

Although status is not a valued part of the architect's role, the external perception of elitism infuses the image such that "...the romantic myth of the asocial, creative architect is particularly strong in some dominant conceptions of architecture" (Jones, 2009, p.2524). Creativity could, therefore, be observed as a means of underpinning this aspect of the image. As discussed in section 4.2, the idea of architecture as a creative art was fundamental to most of the respondents' embryonic understanding of the discipline and continues to be a valued part of the role. Many of the interviewees had observed negative 
perceptions of the creative identities of the architect, particularly the expectation that architects cherish creative output above other, more pragmatic, considerations:

...sometimes architects are seen as always not having efficiency or monetary prudence, it's all about design product and they have to be kept on track. (Architect\#5NZM)

Our image, if you like, comes with lots of baggage. So, we're expensive, we're pushy, we make projects harder, we don't fully understand what the real finances are and we're hard to manage. (Architect\#6UKF)

This introduces the idea that beyond the profession, the creative identities are considered problematic and rather than facilitating projects, makes them more challenging; and are self-referential, rather than valuable. Architect\#7UKM observes that some clients do not take the creative identity of the architect seriously:

...we're in this very interesting marginal state between an artistic pursuit and a professional service. I think the image of the architect is seen with some amusement from some clients.

Through interviews with the contributing architects, a different picture emerges of creativity: design thinking is considered a route to finding solutions rather than causing problems and this argument is explored in greater detail in section 4.5. The data presented here demonstrates that the architect's creativity is attached in the public image to elitism However, this is at odds with the architect's position that creative thinking is a mechanism for solving complex problems.

\subsubsection{Causes of disparity in the image}

To understand how disparity arises between the creative identities, the subjects were asked to discuss ways the external image of the architect is validated and reinforced by architects. The previous section observed how elitism has infused the image of creativity such that the motive for creative output is questioned, some of the respondents considered this is consistent with celebrating the iconic ${ }^{5}$. Samuel observes that, "Starchitects, despite having large global practices, represent only a tiny proportion of architects and employ only a minute percentage of the profession" (Samuel, 2018, p.22).

\footnotetext{
${ }^{5}$ Here, iconic refers to both landmark buildings and architects as described by Jencks in his article The Iconic Building is Here to Stay (2006).
} 
However, Architect\#6UKF comments that more ubiquitous figures within the profession reinforce the status of the architect as distinct, building on the concept of an elite with ideas closely linked to the notion of the hero architect:

...when Foster becomes this Lord of all living things, it sets this bizarre standard that none of us can (or want to) emulate or follow, which is that the architect is the god ... (Architect\#6UKF)

This observation not only sustains the image that the architect is separate but also indicates this particular image does not resonate with the speaker; throwing light on the disparity between the image of the architect and how architects see themselves. This tendency and its effect on the wider architectural culture was introduced in chapter 2 which concluded in observing the evolution of the hero into the star architect. McLeod (1989), Jencks (2006) and Sudjic (2005) scrutinise the cultural value of the icon and its role in treating architecture as a product, by focusing on the aesthetic styling rather than its social and cultural impact. Architect\#8UKM considers the treatment of architecture as a product to be reductive:

It's all these messages to reinforce how simple design is. It resolves itself as a gherkin or some other big gesture, and actually solving a complex problem is much messier, much harder to see.

This observation is supported by Architect\#4NZM who extends the point to include architectural imagery:

I think again probably the internet and the use of images to sell architecture is a problem for architecture because it tries to reduce it down to one simple thing through either two or three perspectives. ... a lot of people think, 'well that's architecture'. But you know it's all of the other stuff that's actually made that.

The implications of this statement are two-fold, first that the amount of work required to resolve a design is minimised and second those aesthetics are treated as an applied veneer rather than the consequence of that design resolution.

\section{Reinforcing the Disparity}

Producing iconic architecture is upheld as an aspiration within the wider architectural culture, and the architect producing it is described in exalted terms such as "rock star" (Architect\#3NZF) and "star architect" (Architect\#2UKM). Samuel further remarks 
"...the Stirling Prize tells us more about the values of architectural culture than about architecture itself and that its opaque judging system devalues architecture in the eyes of the public" (Samuel, 2018, p20). Architects' culture can, therefore, be considered to feed into the image of an elite by perpetuating the significance of the iconic building and the "hero" architect. Stevens (1998) and Till (2009) observe that this tendency commences at the earliest point, where it is reinforced in academies. Several of the respondents concur with this notion, observing how established architects are lionised and star pupils are hand-picked at architecture school:

going through architecture school there was always this perception of, typically the male architects; Mies and Le Corbusier and Louis Kahn...but they were always portrayed as being these people who were charismatic and that would sweep the client off their feet... (Architect\#4NZM).

...there are some people that are teaching architecture who really buy into it and they hand pick people who are going to be the rock stars, but actually there's a whole bunch of people who end up in the real world doing really well but they don't want to be a rock star architect (Architect\#3NZF).

Thus, a culture which focusses on the iconic commences at the beginning of an architect's career, a tendency which informs the way architects address their image. Architect\#2UKM confirms that he plays along with the external image as this is what people expect:

I don't like the idea of the myth of the star architects. I actually really hate it, but at some point you just give in and you just say 'yeah I designed it on the back of [an] envelope' because that's what everyone wants to hear (Architect\#2UKM).

This statement is informative as Architect\#2UKM confirms that he upholds the image of the creative genius where the design is at the tips of his fingers, ready to be sketched, rather than the outcome of a complex process. This accords with Till's comment on "the myth that all great buildings start their life as sketches on napkins in restaurants" (Till, 2009, p.109) the sketch, then, is linked to the concept of genius. By reinforcing elitist and heroic aspects of the image rather than challenging them, architects perpetuate the notion that creativity is innate, and that architectural design is a signature idea rather than a complex process. The observations made by the subjects reinforce the concept that applied design is reductive and obscures the value that architects bring to delivering a 
project. In this way, the gap between the image and the actuality of architectural practice is reinforced.

\section{Reflecting on the Image}

Section 4.2 explored the subject's experience of the architect's image and discussed the disparity between the public image, and the subject's experiences of the actuality of architectural practice. The data presented demonstrates that there is a clear relationship between direct knowledge of the profession and how the architect's role is comprehended. This underlines the significance of image in communicating the architect's role and reveals that architects reinforce an image of elitism, by celebrating the icon; it also supports the notion that architectural design is self-referential and creates challenges. Most of the architects agreed that there is a gap between the public image of the architect and the actuality of practice. The next section explores the implications of this gap and that architects are negotiating the consequential tensions and challenges.

\subsection{TENSIONS AND CHALLENGES}

The preceding section 4.2 explored eight architects' understanding of the image of the architect, in contrast, this section 4.3 discusses frustration within the role. The exploration commences by outlining the architects' experiences of the gap between the image and the actuality of practice and is followed by how the working environment, or context, is changing and how these challenge both the role of the architect and how they work.

\subsubsection{Gap between the image and actuality}

The primary frustration described by the architects interviewed is the time absorbed by tasks, which are necessary to the professional role but fall outside of creative work. This frustration is similar to Sang et al.'s findings that “... respondents had not been aware of the amount of administrative work they would have to undertake" (Sang et al., 2009, p. 317). As discussed in section 4.2, the reasons given by the eight architects for pursuing a career in architecture vary, yet they are united by a common interest in the creative aspects of the role. Similarly, Cohen et al. comment "...the essence of architecture is creative..." (Cohen et al., 2005, p.782). Providing a productive service and working with people also featured as a central motivation amongst four of the architects. 
None of those interviewed reported any interest in the bureaucratic responsibilities required by architecture as a profession, or in the management responsibilities attached to running a business. The majority of interviewees discussed these aspects of the role either as a necessary evil or as something that gets in the way, from their perspective, of the business of architecture. Under Cuff's study into the culture of practice (1991) seven of the eight architects would be categorised as 'fullfledged' with the eighth being "middle years" therefore all have achieved grades of seniority within the profession. According to Cuff, "the full-fledged architect has responsibility for and receives credit for an actual building” (Cuff, 1991, p.148). However, the experience of the architects interviewed suggests that the greater their responsibility, the more they are engaged in administration and management, drawing them away from the design work. This phenomenon becomes a cause of frustration:

I'm still an architect because I want to design buildings, I'm not an architect because I want to be a manager of a business (Architect\#7UKM).

... you get to the point where you're basically managing, just managing the process, managing the people and I'd like to do some architecture (Architect\#3NZF).

... what I'm talking about is the administrative type stuff that's reflective of any business of our size.... I could go a week without drawing anything and i'll go...that's not what i'm here for! (Architect\#5NZM).

Jones (2009) draws on Frampton to emphasise the "contingent nature of architectural production by drawing attention to the role of clients, state regulation, available building and design technology" (Jones, 2009, p.2520). Similarly, Imrie (2007), referring to the "burden of regulation" (p.932) argues that technical and bureaucratic negotiations are a necessary aspect of the architect's interaction with society. The importance of undertaking the administrative and management duties is acknowledged by the interviewees, however, the significant point here is the extent that this aspect of the role is not apparent within the image of the architect:

At architecture school, you know we're taught about the pure design thing which is what we do actually least of... not only are you unequipped from architecture school, you also have a completely warped impression of what you might be doing (Architect\#8UKM).

... what I didn't expect was the bureaucracy, the legal side to do with every aspect of it; dealing with clients, the council, builders, whoever you're dealing with... the bureaucratic side is interesting to learn about, but it's not creative (Architect\#1NZM). 
Likewise, the architects interviewed are not misguided as to the level of autonomy to be expected within professional practice, nevertheless creative ownership of a project is considered an area of tension for five of the eight architects interviewed. In a similar way, Till discusses the temporal nature of architects' buildings, "the reality is that they always enter the social realm as transient objects" (Till, 2009, p.71). Architect\#5NZM observed,

It hasn't happened to me yet, but you get to a stage in your career when your first building is demolished, that's a pretty sobering moment... there's a whole range of signature fit outs that we've done that don't exist anymore. that basically lasted nine years or 12 and then they're gone (Architect\#5NZM).

The issue of authorship is raised by Architect\#8UKM, indicating that design autonomy is also misunderstood:

We as a practice will see a job all the way through from start to finish as often as we can... but, as an individual, it's not often that you get to see it all the way through... so it's kind of imagining something unrealistic to be precious about it: your perfect, untouched project for five years (Architect\#8UKM).

Authorship and autonomy arise from the image of the creative genius and of the hero, the comments above indicate that these notions are at odds with temporality within architecture and introduces the concept of time as a source of tension between the image of the architect and the actuality of practice. Frampton, citing Renzo Piano explains "[architecture] involves a circular process that draws you from an idea to a drawing, from a drawing to an experiment, and from a construction back to an idea again" (Frampton, 2012, p.34). The process is, thus, time-consuming; all of the architects interviewed considered time to be a contradictory element in architectural practice, as there is often a pressure towards efficiency either in a performative way due to client expectations, or economically in the practice's interests:

It took a long time to do things, it does, Architecture takes a long time to do things...it's just expensive isn't it? Drawing things lots of times and figuring them out is just expensive (Architect\#6UKF).

...that's the thing I don't like about architecture, it's so time-based, and you have only so many hours in the day. I find that frustrating; there's so many demands on my time I think that's the biggest thing (Architect\#2UKM).

You need to get traction really quickly, so that time pressure is probably something that going into the profession people probably don't have an awareness of...there is a project pressure to demonstrate value (Architect\#5NZM). 
This feeds into the idea that time is a stretched resource, that lack of time frustrates the opportunities for reflection, curtailing the process, and impacts practice development,

... because you're just moving from one project to the next to the next you don't have time to sit down and go 'what are we doing here?' (Architect\#4NZM).

The discussions show that architects in practice acknowledge that their work is contingent on external influences and control, yet the creative nature of the discipline acts in tension with the more perfunctory aspects of the role; the temporal nature of the built environment and the pressures of resolving the convoluted design process within the constraints of a fixed timeframe. This is similar to findings by Watts (2009), Sturges (2013), Smitheram and Kidd (2019) who also discuss time as a source of tension. Thus, there is a gap between the image of the creative genius and the actuality of the architect as a professional managing a practice and this tension is innate as it occurs between two facets of the architect's identity.

\subsubsection{Changing Context}

The previous section (4.3.1), exploring the gap between the image and actuality focused on "architecture's paradoxical autonomy" (Jones, 2009, p.2521) arising from the tension between conflicting identities of the creative genius and the professional. This section outlines how a changing context challenges the architect's role. The architects were asked to describe experiences of the changing conditions of their practice. The responses varied according to the size of practice and the type of work undertaken. Architects involved with smaller, domestic projects considered the effects of change to concentrate on the technical and bureaucratic requirements of the work, which are becoming increasingly onerous:

The role is changing all the time, it's constantly changing with the technology we use, the bureaucratic and legal stuff and the context is changing so much (Architect\#1NZM).

...the expectations about what we know... and dealing with the council is really difficult...so the amount of work that you have to do just on the periphery, the amount of information that we have to produce now has just increased hugely.

(Architect\#3NZF).

Whereas architects working in a more commercial environment felt the pressure from competition and procurement processes: 
The context we're in is highly competitive and risk-averse so our clients ... all believe in this idea of the competitive tender and the procurement process being what's getting them value for money (Architect\#6UKF).

We win a competition based on the fact we got a low fee and we've done a good design, but it is always an arm's length dialogue-less point to get to that through that procurement gateway (Architect\#8UKM).

Architect\#8UKM continues by observing the implications on cashflow of working in an increasingly competitive environment:

...the margins are nowhere near what they used to be. And I don't think either you or I probably experienced particularly good margins, ever, in business (Architect\#8UKM).

The comments gathered show key effects on practice from a changing context. First is pressure from increasing bureaucracy and technical demands, leading to greater complexity and volume in the architect's work. Second, changes in the economic environment whereby clients focusing on cost and risk management instigates more aggressive competition for work, effecting a downward pressure on fees. The findings, therefore, support the notion that the context is evolving at an increasing rate, making the gap between the image of the architect and actuality of practice a dynamic entity.

\subsubsection{Affects on the role}

Sections 4.3.1 and 4.3.2 outlined the subjects' experiences of tensions within and external challenges to the architect's role respectively; the discussions provide an overview of the types of changes these instigate. Next, the subjects were asked to consider ways the architect's role is changing. This section outlines their responses, focusing on fragmentation, compromise and undermining the architect's position.

\section{Fragmentation}

Section 3.2 outlined the concept of fragmentation which can be understood primarily as "Subdividing a complex task into a variety of minute specialities" (Blau, 1984, p.25), however it is also the outcome of a competitive approach to procurement. Three of the eight architects interviewed were particularly concerned about the effects of fragmentation on the role. The issues were perceived slightly differently between the three, with Architect\#5NZM seeing this as a pragmatic response by contractors to 
delivery challenges. Nevertheless, he observes the narrowing and erosion of the architect's role:

The commercial market has got some significant issues around delivery ... with that, the delivery sort of projects start being provided by contractors as a way of trying to resolve the issue. But there certainly is a specialisation going on and project managers are starting to try to expand their remit and the interface with the client (Architect\#5NZM).

Architect\#6UKF considers fragmentation to be a trend which is leading to the profession becoming de-skilled. This position is drawn from the observation that separating out roles means the consequences of design decisions are not realised when earlier-stage designers have no involvement in later processes:

... if the architects don't have the construction experience, because they're not seeing it through onto site, when they're drawing at the early stage they don't really know what they're drawing. And so we're building in an ignorance (Architect\#6UKF).

This comment relates to Cuff who predicts “...the deskilling of labo[u]r as each worker is expected to perform a narrower range of tasks" (Cuff, 1992, p.207). Architect \#8UKM, however, comments on the wider impact of fragmentation:

That's the thing that can be so corrosive, is the fragmentation. You find you have to compete just to do the feasibility study...then you inevitably get to re-tender at planning and re-tender for delivery. Fragmentation can be quite corrosive, so it's navigating that as a practice (Architect\#8UKM).

Thus, the architects experienced fragmentation as challenging the role, effecting a loss of skills and impacting practices' workflow. The concept of fragmentation is incongruous with the image of the architect; it contradicts the notion of autonomy and, in particular, the image of the overarching role of the polymath. The comments on the effects of fragmentation reveal that the architects consider that this over-arching role is changing.

\section{Compromise}

The preceding discussion explicates how increasing complexity in projects leads to fragmentation and signifies the architect's over-arching role is changing. A second challenge reported by the architects, is compromise. Ahuja et al. consider the effects of working within a team or through complex sets of project criteria, "architects nevertheless struggle against changes initiated by others, which are viewed as threats to 
the aesthetic integrity of the design" (Ahuja et al., 2017, p.9). In a similar way, Architect\#4NZM described it as relinquishing autonomy within the design process to accommodate the client or design team's input:

...you're always having to discuss and debate... it takes a lot of energy each day to constantly have to present your case, to listen to somebody else's case to then debate and resolve to try and move forward (Architect\#4NZM).

However, the majority of observations relate to budgetary or pragmatic constraints which lead to a compromise in the design outcome:

... we think we can [make a difference] and I think the reality is we can't, because of the problems I've identified: the mechanisms that we work within; the masters we serve and the economic premise behind pretty much everything we do (Architect\#8UKM).

Certainly we would have to work very hard to justify some of the more sophisticated European facade systems, for example. So, both in terms of just the square metre material cost, but equally the technical installation expertise to do it (Architect\#5NZM).

We also did quite a lot of work with a big developer in town ...they do have quite strong views of what they want, which I think is more driven by them talking to estate agents who then say, this is what's going to sell (Architect\#4NZM).

In a contrasting position, Architect\#1NZM acknowledges that compromise can occur to accommodate a budget, however, his experience is of a more fluid transaction:

...you get that range of clients as well and they go "well I want to spend this but this is what I want" that's always the starting point, and then you go through the process and then you get the price and it's a shock but then they go "but I actually want that so we're going to do it" (Architect\#1NZM).

This comment is interesting for two reasons, first it indicates the importance of process and transparent dialogue with the client in managing compromise. Second, the situation recounted by Architect\#1NZ describes a simple project structure, represented by a direct connection between two parties; architect and client whereas the situation outlined by Architect\#4NZM, describes a more complex team structure. This observation underlines the point that increasing complexity in projects leads to diffusion in authorship and compromise challenges the image of autonomy. 


\section{Undermining}

The previous sections explore how fragmentation has led to increasing compromise, which in turn diffuses authorship of a design concept to the wider project team. This links to Section 3.2, which discusses how the architect's role is changing. Where fragmentation and compromise directly challenge the autonomous identities, the architect's status and, thus, the concept of an elite are also challenged. Caven and Diop (2012) discuss the intrinsic rewards of "prestige and value to society" (Caven \& Diop, 2012 , p.514) as a reason why people remain in the profession and, thus, challenges to this status by "less qualified individuals such as project managers" (Caven \& Diop, 2012, p.519) has an undermining effect on the profession. The participants in this research concur with this observation, outlining several factors for the phenomenon. Three architects observed how reducing design into an aesthetic overlay leaves strategic decisions to others:

I think a lot of the opportunity to influence business or institutions or placemaking is being done by surveyors and investors and planners: all the people who have no significant design impact, and no vested interest in the long-term outcome (Architect\#8UKM).

It's not that we're sniffy about doing design and build ... they just want you to just style the building and I think at that point it's not a critical process. It's undermining some of the main design ideas for either short-sighted ideas of project management or pennypinching (Architect\#7UKM).

Architect\#3NZF, who works for domestic clients, felt that her role was being undermined due to cost cutting, where a two-tier system emerges between bespoke architect-designed projects and cheaper off-the-shelf contractor-built projects, indicating competition from other facets of the building industry as a factor:

... there's been an undermining in New Zealand because of DIY and building companies... some people who wanted to knock down their house and build a new house...they went to a house builder and they were like we don't think we can afford an architecturally designed house (Architect\#1NZM).

Therefore, fragmentation and competition can be seen to undermine the architect's role. Undermining can be understood to occur when facets of the role are undertaken by nonarchitectural service providers, or by separating work-stages into packages. The implications of undermining are that the influence of the architect and therefore status are being eroded; further, a loss of skills results from narrowing of the architect's scope of 
services. Undermining, therefore, challenges the image of the architect as an elite, and within that, its value.

Value

The preceding discussion situated the challenges to the architect's role within the context of fragmentation, compromise and undermining, issues which can be considered to controvert facets of the image (polymath, genius, hero and elite). This also erodes the notions of autonomy and status. Caven and Diop's (2012) research into the rewards associated with an architect's career investigates value as an intrinsic reward of the profession. Value in this sense can refer to architects' sense of esteem or financial worth. This section explores the notion of value, building on the previously discussed themes of fragmentation and undermining.

When discussing the challenges to their role, the extent to which the architects felt their contribution was appreciated was a factor for some. Architects working with domestic clients reported that their clients appreciate professional support:

I was taking photos of things that were special to [the client], things she wanted to put on display or that showed the feeling she wanted in her house and she was crying by the end of it...she was like 'thank you for listening to me' (Architect\#3NZF).

In contrast, the experiences of architects working in more commercial settings was that architectural design is less valued:

I think personally the architect has a huge aesthetic responsibility as well and I think that that's often very undervalued ... it's very marginalised as a discussion about what buildings look like (Architect\#7UKM).

The idea is to communicate to the outside world that we have value and that our image needs to show that there is value in what we do and I think we have, as well, eroded that (Architect\#6UKF).

These statements indicate that architectural design is not valued as an isolated activity. Bernstein concurs, observing, "[the architect's] work is often characteri[s]ed and paid for as if it were any other lowest-cost commodity in the construction supply chain" (Bernstein, 2015, p.210). Additionally, the architect's comments indicate that they are not adequately communicating the value of the role. This is the essence of Samuel's research into architect's value, "part of the problem for architects is that they have not played an adequate role in the making of the bald statistics through which their value is perceived" 
(Samuel, 2018, p.96). When the contribution architects make is not considered of value, the financial worth of the role is also challenged.

Architecture is acknowledged within the profession to be poorly paid, this position is supported by two of the architects interviewed, who observe:

Guys on construction sites get paid more than a lot of architects...it's very difficult to maintain that for very long (Architect\#2UKM).

...unfortunately, because it's not valued very well in the UK it's not a particularly wellpaid profession it is rewarding in other ways isn't it? (Architect\#7UKM).

This final comment ties the two concepts of value together, that financial worth and the esteem of the role are linked. The comment is also similar to Caven et al., who observe that architects have "...poor salaries in relation to other professions..." (Caven et al., 2012, p.373). However, Caven and Diop (2012) suggest intrinsic value is a key factor for architects remaining in the profession, which also concurs with Architect\#7UKM's statement. These conflicting ideas, therefore, reveal the problematic interactions within the concept of value. Fees agreed through competition can be insufficient to achieve both a comprehensive design process and a profitable practice, suggesting that the circuitous and open-ended aspects of the design process are not financially accounted for within competitive fee negotiations. This is supported by the architects' experiences.

\subsubsection{Review of tensions and challenges}

All eight of the architects interviewed identified innate tensions between the architect's creative identity and professional obligations, indicating that there is a gap between these aspects of the image and the actuality of working as an architect in practice. Further discussion reveals how the working context is evolving to instigate challenges from greater technical and bureaucratic complexity and increasing volumes of work. Architects reported that competition is leading to a more challenging fee environment. The interviews illustrate that the images of autonomy, creative genius and hero are diminishing as projects grow in complexity. Most noted themes amongst the interviewees were issues with fragmentation, compromise and a sense of undermining; the architects who reported these challenges were working with multifaceted client structures where lines of communication are more diffuse. Conversely, the architects who work with predominantly domestic, or simple, client structures, where the dialogue 
between client and architect is direct, still considered their services to be valued. The next section 4.4 will outline how the architects are negotiating tensions and challenges, exploring prospects for the profession and how they foresee it evolving alongside the working context.

\subsection{NEGOTIATION}

The preceding section 4.3 explored eight architects' understanding of the tensions and challenges which their work is subject to and the affect that these have on the architect's role. The discussions uncovered the innate tension between facets of the image of the architect which creates a gap between the image and the actuality of practice.

Additionally, challenges emerging from a changing context were identified in the form of increasingly complex projects, project structures and evolving technical and bureaucratic requirements. Further, section 4.3 identified that the gap is dynamic, expanding as the context continues to evolve and that these tensions and challenges result from fragmentation, compromise and undermining. This section 4.4 discusses how the architects interviewed are negotiating this gap to create practices fit for purpose in the contemporary context. It will explore how architects are negotiating tension within the image and responding to the external challenges. The exploration commences by examining ways in which architects are challenging the prevailing image. The way that the architects negotiate the external challenges follow, observing how their practices are changing to redefine how they work.

\subsubsection{Challenging the image}

Section 4.3 discusses aspects of the prevailing image of the architect which are at odds with the actuality of practice. Most notably, components of the creative genius and hero are identified as particularly problematic within the contemporary context when associated with notions of the iconic, elitism and the concept of autonomy. The architects interviewed express disquiet with these ideas and explained how they work in consideration of the changing context by regarding design as a collaborative process. Architect\#4NZM rejected the notion that iconic buildings can be produced in isolation; in his experience, a building has to earn cultural significance to be considered iconic.

Iconic is something that is not just the architecture alone when you've just built it, It's the cultural and political setting and everything else that then people buy into...(Architect\#4NZM). 
Architect\#8UKM also rejects iconic architecture as an objective, in his case the design process is more important than the pursuit of a singular design aesthetic:

I get really cross with, form making architecture ... It doesn't need to be an aesthetic at all. If it's there's building with a great diagram it doesn't matter, in many ways, how it looks (Architect\#8UKM).

Styhre \& Gluch, similarly describe architecture as “...fundamentally collaborative and includes tight communication with various stakeholders including clients, contractors, and end-users" (Styhre \& Gluch, 2009, p. 224). Architect\#7UKM builds on the notion that process is more significant than a focus on aesthetic but considers that resolving the complex strands within a project through the design process leads to an aesthetic conclusion:

... synthesising the problems of the commercial realities of the site, but also the social needs of an area and I think that good architecture can negotiate that and provide an aesthetic, cultural result as well (Architect\#7UKM).

Consequently, these comments contest "...the mythology of the sole architect as heroauthor" (Schneider \& Till, 2009, p.97) instead, these architects consider the design process, as a resolution of complex external factors, to be paramount. As discussed in chapter 2, arrogance, is also associated with the image of the hero. Three of the architects interviewed commented on how they directly challenge this disobliging aspect of the image:

...what's the point of being [arrogant]? I want to be able to work with people, I'm more about providing a really good service and being accessible (Architect\#3NZF).

I think I try to be quite pragmatic about stuff. I like the idea of being creative without being [obnoxious] (Architect\#2UKM).

[A client] is very interested in working with me as he sees me as different. He thinks I'm authentic and he's worried that there's a lack of authenticity in the profession (Architect\#6UKF).

The comments indicate these architects are adopting an open and collaborative approach to practice, which could be observed as a response to the architect's evolved role, not as a dominant figure, but a collaborative character. Many of the architects interviewed were 
not possessive about the origin of the creative idea but see the benefits of working as part of a team to generate a creative outcome.

...it doesn't matter where the design idea comes from in the team, if it's the idea that we should run with, we'll run with it...So, there's a lot of design ambition but there's not a lot of ego (Architect\#5NZM).

I think the style and the idea that design and high design is the pinnacle is of course nuts isn't it? Because it takes a whole load of us to do this well (Architect\#6UKF).

[my client's] changed the design so that's been a real collaboration, he'd actually sit at my desk with me and we'd model things together and that's really nice, it's a good process (Architect\#3NZF).

The above comment by Architect\#3NZF is interesting for two reasons, first it demonstrates that in her experience, a collaborative process can be productive and rewarding, second it shows the importance of the client role. In this example client engagement is key to developing a productive design process; this position is also reflected by others:

...it's just simply about sitting down quietly, calmly with the client and working through a number of complex issues again and again and again to come up with whatever that design is...that makes the design ultimately better. And it's more fit for purpose (Architect\#4NZM).

... if you talk to people about their work and if they're genuinely interested in it then you might want to do the work for them (Architect\#1NZM).

...that's part of the process side of things in terms of not being dogmatic about a design formula, but that we bring clients along; it's a method, we bring the client along that design discovery process so we engage with them, we don't dictate to them (Architect\#5NZM).

Whereas other architects simply recognised that gaining the client's assent is key, whether it is via the design process or a synergy of goals:

I don't think good architecture has to cost a lot of money, I think some of our earlier projects demonstrate that and I think good architecture needs an alignment of values with clients (Architect\#7UKM).

...it's always hard to justify the long process that we try and get through to get the best outcome for the client because it's costly and you have to persuade them that they are procuring for long term value and design quality will come through a process (Architect\#8UKM). 
The importance of the client role as expressed by the architects above challenges the identities of the creative genius and the hero, where creativity is synonymous with autonomy. Likewise, Imrie and Street, citing Edwards, observe “....architects, work 'in and between work settings and interact with other practitioners and clients to negotiate interpretation of tasks and ways of accomplishing them"' (Imrie \& Street, 2014, p.726). By adopting collaborative and accessible ways of working, the architects interviewed challenge the aspects of the image which are at odds with the architect's role within the emerging context. Section 4.3.1 outlines the inherent tension between the image and the actuality brought about by the contradictions between two facets of the architect's identity: the creative genius and the professional. The architects who challenged the problematic aspects of the creative genius, by adopting collaborative and accessible ways of working, can be observed to be negotiating the gap between the image and actualities of architectural practice. A second way the architects are negotiating this gap is through a strategic approach to practice structure and in attracting work.

\subsubsection{Strategic thinking}

Section 4.3.2 discussed the changing working context and how this exerts an external pressure on the architect's role. This section (4.4.2) explores strategies adopted by the architects to anticipate the changing context and negotiate challenges to their work. Architect\#7UKM describes how his practice is structured to provide resourcing contingencies to allow for unanticipated changes to workflow:

...just because you are awarded or you win a commission or you invent a project from absolutely nothing with a client, it's no guarantee that it actually will get built, so you have to predict a certain amount of redundancy of projects that will never happen. That's why the practice is of a certain scale because we're sort of overlapping opportunities...(Architect\#7UKM).

An alternative approach is taken by Architect\#5NZM who describes how his practice size has expanded in direct response to workflow and that their strategy is to treat this as an opportunity for centrifugal growth:

...it has grown as a result of the project work meaning that we have to. And then once we had grown trusting that we can continue that workflow to suit the size of the practice... So, we talk about this upward spiral ... that attracts better clients and attracts even better staff over time. So it all just positively informs itself (Architect\#5NZM). 
Architect\#8UKM describes a completely different strategy for responding to external challenges; his practice is structured as a co-operative so that each member is empowered to make management decisions, this is considered to create versatility in responding to the evolving context:

Our flat, employee-owned structure leads to [entrepreneurialism] quite well. Everyone in the practice is empowered to speak at things, direct the sort of work we get, represent the practice in whatever they want to do really (Architect\#8UKM).

Winch and Schneider consider that "a crucial element of effective strategic management to explore how a practice may extend or reconceive itself' (Winch \& Schneider, 1993, p.933). Of additional interest is practice size; Architect\#7UKM and Architect\#8UKM's practices are considered to be medium-sized practices (RIBA, 2018), whereas Architect\#5NZM's practice is considered to be large. Within Gutman (1988), Cuff (1991, 1992), Symes et al. (1995) and Jamieson's (2011) research, these types of design practice are considered vulnerable to the service orientated firms. However, the architects' alternative approaches to practice organisation demonstrate how they are responding strategically to the changing context and they are doing so creatively.

This comparison is significant when observing Architect\#1NZM, Architect\#2UKM, and Architect\#3NZF who all run what are considered to be small practices. Within Winch and Schneider's study, to undertake creative work, “...small flexible and responsive organizations are preferred" (Winch \& Schneider, 1993, p.931). Winch and Schneider (1993) proposed small practices might specialise to enable expertise to be developed along with a reputation within a particular field of work. These architects confirm that the ways their practices respond to the changing context is to carve out a practice which reflects the aspects of the role they most value:

I've either steered away from those kinds of clients, where finance is a key thing, or through luck I've managed to find clients who are invested in the architecture (Architect\#1NZM).

The thing that we have always talked about is that we would only want to work for people that we want to work for, and so most of the time we get clients that we really relate to...we've also created a practice which is flexible around our family as well, which for us is really important... (Architect\#3NZF).

I don't want to push this thing faster than I think its natural pace is and I think, yeah, the practice has been developing a lot...I was actually trying to get some more commercial stuff, but I'm not going to throw everything away for that (Architect\#2UKM). 
This suggests that the strategic responses reported by the subjects is informed by challenges from the changing context but is also dependent on practice size; "In response to the changing circumstances, firms have tended to become either larger and more comprehensive or smaller and more specialized..." (Cuff, 1992, p.204). The variety of practice strategies for responding to the challenges also demonstrate that this is a creative response to the challenges of running a practice in the contemporary context. A further demonstration of this point is through comments made by three of the architects over their strategies for acquiring new work within the context of increasing competition and complex procurement routes. Architect\#6UKF explains how she has developed a strategy for creating opportunities through a specialism in neighbourhood design, as a mechanism for meeting with potential clients:

[1] do the research and then I can get in front of big developers...I'm starting to realise how to play the system and its really hard, but I'm getting in front of everybody (Architect\#6UKF).

This creative approach to generating work is similar to Hill's prediction, "instead design stratifies along a different axis...performed by strategic generalists and synthesists, engaged in cultural invention" (Hill, 2012, p.13). Whereas Architect\#7UKM's strategy is to work closely with clients to create projects but through a track record for winning design competitions. It is a strategy similar to Winch and Schneider's description of how practices with strong ambition move into a strong ideas model "the route to the strong ideas strategy is often through winning an architectural competition" (Winch \& Schneider, 1993, p. 931). Architect\#7UKM explains that these approaches accelerate the practice's work opportunities:

We're quite experimentative about the way we get new business and I think that's central to our business plan. But what's really interesting about architectural competitions I think is for us, to begin with, it was a really important way of leapfrogging our place in the queue.

Architect\#8UKM describes his strategy for circumventing complex procurement processes by developing a network of contacts whom he can directly lobby for new work:

What we prefer to do is have interesting conversations with people that lead to more interesting people and start to get into a feasibility type of conversation which gets us into the conversation earlier than we would otherwise do if we waited for a project 
manager to run a two-stage competition to get there. So we think relationships are a really important part of the practice... people move around and you want to retain those relationships (Architect\#8UKM).

This strategy accords with Samuel, who identifies networks as a template for future resilient forms of practice, “...practices need to form collaborative partnerships with others, a process that brings long- term network dividends" (Samuel, 2018, p.192). Thus, the findings demonstrate how the architects are using creative judgement differently to adopt strategic approaches to negotiate the challenges from an increasingly competitive working context. The next two sections (4.4.3 and 4.4.4) will explore in greater detail how architects are using creative judgement to negotiate the effects of fragmentation and the undermining of their role.

\subsubsection{Diversifying and Specialising}

The previous section (4.4.2) outlined how architects are thinking creatively to form strategies for responding to the changing context both in the ways they have structured their practices but also in the ways they acquire work. This section explores how architects negotiate the effect of fragmentation on their role, either through diversifying "laterally into other building disciplines... or diversify outside construction into areas such as design” or specialising, “...to establish reputation,” (Winch \& Schneider, 1993, p.933). The architects were asked to outline the ways their practice's work is adapting to external challenges to their role. Half of the architects interviewed identify that their practice was diversifying as a way of exploring new streams of work, either by project type or by using skills in negotiating and innovating to explore new areas of work. Architect\#3NZF commented that to grow the practice beyond their current domestic portfolio, they would need to diversify the type of work they do. She observed that creative judgement as part of a design process transcends different types of work, therefore Architect\#3NZF believes her established skills as an architect would enable this transition:

If we change the type of work we do there would probably be a learning curve... but I think the process of architecture is the same... I actually would like to try some other things that are not necessarily architecture but more design...(Architect\#3NZF).

Other architects also recognise that the wide range of skills facilitate the transition into new areas of work. This suggests a value in the image of the architect as polymath, but 
their decision to diversify was described as a way of generating a workflow within a competitive environment:

In New Zealand, because it's a small market, you can't focus on one project type or one client type so it's a much more diverse kind of practice here. You have to be a generalist... you really have to spread that net as wide as you can (Architect\#5NZM).

...we've had such an unusual mixture of projects, opportunities because we've had to do that because we grew the practice out of the last recession so we had to take unusual opportunities (Architect\#7UKM).

Building on these ideas for growing a practice and negotiating a competitive market, a third reason architects gave for diversifying relates more to their concerns about the future of the profession. Two of the architects interviewed were particularly focused on how fragmentation is changing the role. These architects consider how their work can evolve to negotiate increasing challenges to the architect's role:

... it will increasingly, I think, go into big contractors...if we're interested in solving complex problems and accept that it might not look like buildings but involve design thinking then I think we could be in a really exciting profession and that's where the research and the open-minded exploratory business development is pivotal (Architect\#8UKM).

I think the profession, if it is a profession, needs to totally regenerate into a profession where we see what we can bring, we need to really galvanise what we can bring as culturally and artistically and strategically led, rather than just technical service providers, because I think there's going to be Google plug-ins for many of the other things that architects do in a few years (Architect\#7UKM).

These comments reveal two things, first is the understanding that other professions will continue to compete for overlapping services and second, that the adaptive and creative identity of the architect is key to the profession's ability to negotiate these challenges by diversifying. These observations are similar to Tombesi (2012) who foresees a valuable role for architects as coordinators across a range of skill bases within emerging, fragmented work models. Another interesting point to observe is that, except for Architect\#3NZF who describes diversification as a way of growing her small practice, the architects who diversify have medium and large practices. Three of the architects interviewed took a contrary strategy towards specialism, run small practices, in the case of these practices, their strategy is driven by a decision to target a particular stream of work and develop a unique selling point: 
What I did when I realised we had to get away from doing back extensions, when we came together 5 years ago, was to start [a] piece of research ... I thought (and this bit of it worked brilliantly) that I'd get exposure and contact with developers and clients (Architect\#6UKF).

Every practice has its ways of doing things and so I've tried to make more of it so these are research areas, but that is part of the creative process. You've got these fields of stuff you do and it's much easier to start with those and reinvent them each time than start with nothing (Architect\#2UKM).

These architects' comments reveal that they are shaping their practice by focusing on a specific type of work and way of working, which resonates with the discussion in section 4.4.2 whereby practice size influences the strategic approach taken to negotiate the changing context. Their comments also introduce the role that research, and critical practice plays in how architects negotiate the changing context by exploring new areas of work.

\subsubsection{Research and Critical Practice}

The preceding sections 4.4.2 and 4.4.3 discussed how architects utilise creative judgement and strategic thinking to negotiate the challenges to their practice from the evolving context, introducing research and critical practice as a further strand for exploration. Cuff (1991), Gloster (2015) and Samuel (2018) identify the opportunities for evolving the discipline through a closer alignment of research and practice. Hyde (2012), on the other hand, explores the opportunities that architects are taking by branching beyond the established realms of practice and pedagogy to forge new roles and innovating to find fresh streams of work. During the interviews, the architects were asked to consider any ways they are using their skills, creative judgement and strategic thinking, to evolve their practice. The architects considered that continued development of knowledge is an inherent part of their practice, tying into Schön's (1995) conception of the reflective practitioner:

That's one of the things actually that makes the profession engaging is that you are always learning. You know you don't just do eight years of training and then trade on that or coast on that for the rest of your career, so it is always engaging (Architect\#5NZM). 
However, a further reason was given for undertaking research or undertaking critical practice is in forging a new role for the architect. As outlined in section 4.4.3 critical practice refers to theoretical development which goes beyond reflective practice to undertake "a rigorous forward movement, capable of producing new concepts out of the hard logic of architecture's working procedures." (Allen, 2009, p.xii). By offering services which have an enhanced value within the contemporary context, architects undertake research to develop specific technical knowledge. The architects interviewed outlined three reasons for undertaking research or critical practice, to add an additional layer to their practice. First was self-motivated, that is pursuing a specific interest, or to underpin a specific design philosophy. These ideas convey the notion of architects exploring the intrinsic value of their work:

I'm thinking about setting up a separate wing of the company, a non-fiscally rewarding wing that deals with one-off stuff that you really are exploring, probably largely around environmental stuff (Architect\#1NZM).

...when we do these [CNC self-build projects] where we're really stepping in...where we're taking all the responsibility, then in a way I think that's something that architects can do...I think the other advantage of having these streams of interest or research is that I think, actually, it's quite fun (Architect\#2UKM).

... we're thinking about how spaces are occupied and how they can also be adapted over time...some clients and some people do like the idea of a fixed image that you've locked in, but the reality is that your buildings always adapt and change no matter what (Architect\#4NZM).

So I think you either subscribe to the fact that some processes may yield quick results or you invest in architectural ideas in small projects that get embedded into your design methodology in larger projects later on. So you're sort of investing in your own critical practice (Architect\#7UKM).

The second motivation architects gave for undertaking research or critical practice was as a vehicle for demonstrating value in the design process and validating design solutions. Samuel underlines the importance of research for the validating the architect's role, "If architects can evidence their value in a manner that the rest of the world understands...their value becomes self- evident" (Samuel, 2018, p.238). Four of the architects reported how they were engaging in practice-based research:

There's a big stream of work which is really just starting about how do you prove a social return on investment, for example. That's to do with how you prove the value of 
good design and therefore architects...then we start to boost the leverage that designers can have or should have (Architect\#8UKM).

... with the offices that we designed recently...they do all these post occupancy surveys. I mean one of the most interesting ones with the health and wellbeing stuff was that the sickness rates dramatically dropped (Architect\#2UKM).

...we were doing that research to actually help to give us a justification in terms of our conversations with client groups... You can use it as metrics-based evidence with clients who may have reservations but otherwise you're just submitting a subjective design opinion (Architect\#5NZM).

...its just a better way of designing [socially]. It's how we should be massively considering housing developments. And for some architects, they want to work like that anyway but they don't have the research and data to back it up. So they can use my research to prove that their design is superior...(Architect\#6UKF).

The third impetus takes the form of prospecting; exploring new fields where architectural skills can be invested to either redefine the role or create new areas of work. Salomon categorises this as, “...design-oriented practices stress the need to develop new methods for creating architectural projects" (Salomon, 2012, p.438). The following architects describe the core skills which might facilitate innovation:

If, through the process, I have greater command of the economics and policy dimension of what we do, I can apply that in different theatres than pure construction... no one understands design in government, and no one understands economics in architectural practice; there's just so little overlap in complex problem solving and skills allocated to the wrong places (Architect\#8UKM).

I think the only way the profession can survive is if architecture is taught values of good judgement. Synthesising aesthetic, cultural, society and technical things (Architect\#7UKM).

The architects, therefore, reveal how they are responding to the challenges from the evolving context, whereby fragmentation and undermining lead to erosion and a loss of value in the role. By utilising their skills in strategic thinking and creativity, they are focusing on innovation and judgement rather than the technical delivery aspects which are, in any event, being offered by others. 


\subsubsection{Review of negotiation}

Through this section (4.4), we can observe how all eight of the architects interviewed are negotiating the gap between the image and the actuality of architectural practice. First, this section explored how the architects are challenging the prevailing image through how they work; disputing the aspects which are no longer pertinent to the contemporary context. These include the concepts of autonomy and elitism and ideas surrounding the iconic. Further, through collaborative working, particularly through client relationships, the architects explicate design as a process beyond an aesthetic overlay, which involves multiple strands of complex problem-solving. Second, this section revealed how the architects are reinforcing the aspects of the image that they hold to be valuable and which they consider give them the tools to re-define their role and explore new realms of work. These include the concept of the polymath, creativity and judgement which facilitate strategic thinking and form the foundations from which they are adapting their practices. Finally, this section (4.5) demonstrated how the architects are structuring their practices in response to changing workflow; adjusting their work focus in response to fragmentation; strategising to acquire work in an increasingly competitive market and evolving to offer technical knowledge and skills which enhance the value of their role within the contemporary context.

\subsection{CONCLUDING DISCUSSIONS}

There are common observations and experiences across the architects who have contributed to this research; however, their solutions for negotiating the tension and challenges in practice are divergent. The first conclusion from the study is the external image of the architect is diffuse and the potency of the architect as a professional, or expert, has been eroded. This chapter has demonstrated how, in the contemporary context, an emphasis on the image has led to a focus on product rather than process, but also that a layer of mystification between the external image and the actuality of the architect's role obscures the gap between these two entities.

Second, the study reveals that architects consider the complexities of the design process are what brings value to the architect's role, as it is this process which ensures a successful project. Focusing on the image, rather than the process misrepresents architectural design as something to be applied, a luxury item rather than fundamentally important. For this reason, a majority of the architects spoken to reject the notion of the star architect: they consider the concept is problematic as it simultaneously invokes an 
image of glamour, celebrity, single-mindedness and arrogance, but is also "inauthentic" (Architect\#6UKF). The lack of clarity in the image of the architect, but the emphasis on this image rather than the innate value of the underlying process are all interlinked and act to create tension between the image of the architect and the actuality of practice. Focusing on the process is, therefore, key to communicating the value of the architects' role.

Thirdly, this chapter describes how the traditional image of the "hand of the architect" (Architect\#4NZM) as an uncompromising and authoritative figure is no longer valid. Instead, architects work subtly using compromise, strategic thinking and problemsolving in an intricate and thoughtful way to deliver projects. The study reveals that the role requires a range of skills, which are not inherently linked to the image of the architect: these include administrative and business acumen; psychoanalysis (and counselling) of users or clients; and an aptitude for negotiating with multiple parties. These particular skills come from the service nature of the role, whereby the architect is working in the service of a client and under a duty of care to society as a whole. In the absence of an involved and enlightened client, the seminal project cannot exist, therefore, architects work through diplomacy and advocacy, facilitating client engagement in the process, thus, disputing the pertinence of the image of the autonomous 'hero' architect.

Finally, this chapter observes how the multiple layers of skills essential to executing the role of an architect are also exploited to devise new ways of working, explore design through research and build networks to adapt architectural practice in response to changing context. However, the diverse approaches ".. reveal the fragmented state of architectural practice" (Salomon, 2012, p.441) signifying that negotiation is occurring on an individual basis rather than as a co-ordinated industrybased approach. In this regard, the image of the architect is being challenged internally as well as from external forces as a means of negotiating the gap between the image and actuality of architectural practice. 


\section{CHAPTER 5}

\section{Discussion and Conclusion}

The introduction of this thesis opened with the statement that the role of the architect is changing. The study then followed the argument that although there is an enduring social value to the architect's role, within the contemporary context, this is not explicitly recognised. Chapter 2 commenced the research by discussing the image of the architect and how this has evolved as a response to changing context. Then, chapter 3 explored how the image has become disconnected from the actuality of the architect's role in practice, observing that the image has not evolved in step with an exponentially changing context and how this has created tension. Chapter 3 reviewed literature which discussed this phenomenon but also literature which proposes how architects can work differently to negotiate the emerging challenges to the role. Chapter 4, then presented the findings from the qualitative research which tested the theoretical findings, through the direct experience of architects working in design practices.

This final chapter comprises both a discussion on the findings of the theoretical and qualitative research together with the conclusion to the thesis. The findings which have emerged from chapters 2 to 4 are interwoven, therefore, the discussion will return to the three key themes outlined in the introduction for its structure: image, context and negotiation. The conclusion also considers how this investigation might be expanded and suggests strands of investigation which could grow out of the research.

\subsection{IMAGE}

Overview of the Study

The first of three themes investigated through this research relates to the image of the architect and how this has been built as a layering of identities over time. Chapter 2 discussed literature which locates the changes in the architect's image against historic economic, political and social change. By overlaying the changes, connections between the evolving architect's image and changing context can be observed. The earliest developments in the architect's image take place over centuries, therefore change is incremental. The study also reveals that within the last century, the context is changing 
exponentially, the image has not evolved in-step and a gap between the architect's image and the actuality of practice has emerged. Chapters 3 and 4 explored the implications of this gap, identifying that the contemporary image of the architect is connected to a celebration of icons, focusing attention on applied aesthetics rather than the complex processes involved in architectural work. The literature review also considered texts which explore architectural culture to explicate how the image is self-perpetuating and this literature holds clues as to why the architect's image is not evolving in line with the context.

\section{Discussion on Image}

The study tracks the way the image evolved as a layering of identities. Through this, three points are clear. First is consistency; the concept of the highly skilled polymath runs throughout the differing images, thus, underlining the significance of the architect's identity as highly-skilled across multiple fields of knowledge. Second, the core identity of the polymath is augmented by the early focus on the liberal arts, creating separation between manual work and artistic pursuits. This signifies an aspiration for status, to set the architect apart from the craftsman, but it also shapes the specific skills and qualities which become ascribed to the architect through the developing image. The skills and qualities which can thus be considered to delineate the polymathic identity are creative thinking, judgement, taste, ethics and morality. Third, the image is mutable; by isolating the image from the core identity of the polymath and the gradual delineation of skills, the images of the artist, genius, gentleman, hero and star can be observed as disconnected concepts which are contingent on the immediate context. Consequently, tracing the development of the image shows how the image of the architect is adaptive, responding to the immediate working context, whereas the tacit qualities and skills have developed incrementally as a layered process of evolution. This shows an innovative and flexible aspect to the discipline, but also illustrates the reductive nature of the image.

Chapter 3 demonstrates how architectural culture reinforces the prevailing image inwardly. This process can be observed to have two effects; first, it advertises an aspirational position for the role which does not necessarily reflect the everyday experiences of architects working in practice. Second, it distils the essence of the architect into a single headline concept; projecting a specific image outwardly obscures the complex range of skills offered. These effects, therefore, illustrate how a selfperpetuated image is a factor in the tension between image and actuality. The research 
section explored these effects further through conversations with architects working in practice. According to the participants' experiences, comprehension of the architect's role is linked to the level of exposure to the profession before entering the profession, illustrating how the extents of the role are unclear to those outside of architecture.

Additionally, Chapter 4 also demonstrates the impact of the image. Many of the architects interviewed did not consider they appreciated the full implications of the role until they commenced work in practice, demonstrating that the image is unclear, even during academic study. Therefore, academic institutions reinforce the prevailing image. Finally, the architects interviewed were aware of the implications of the image, but most considered this did not represent the work they undertake; they considered the process of solving complex problems through design thinking and negotiation with others to be of greater significance to their work. Consequently, some of the architects interviewed thought the image was less important than clarity in the role itself. From this, we can conclude that the image of the architect is not pertinent to the day-to-day work architects perform and, in some respects, it obscures the complexity of the design process and the range of skills required to fulfil the role.

\subsection{CONTEXT}

Overview of the Study

Chapter 3 identified and analysed texts which discuss the contemporary challenges to architectural practice, the qualitative research built on this information, gathering the experiences from architects working in practice. The greatest challenge arises from technological advances and increasing complexity within projects. These phenomena have stimulated growth in specialist services offered from within the construction industry, an issue which has occurred simultaneously to the changing authority of the architect. The concept of architects' autonomy and the notion of architects as an elite are demonstrated to be misguided concepts within the contemporary context, whereby collaboration and compromise are required through design processes and fragmentation and undermining of the architect's role challenge its intrinsic value.

\section{Discussion on Context}

Analysis into the evolving image shows little change occurred in the context up until the Enlightenment; thereafter change occurred with increasing rapidity and since the twentieth century, this change has been exponential. It is not unreasonable to predict that this 
trajectory will continue and therefore if the role is to continue, it must adapt in line with, or in advance of, the developing context. This thesis demonstrates that the image is not changing in step with the demands of context, leading to tension between the image and actuality of practice.

Chapter 3 touches on the concept of "overtraining” as outlined by Larsson (1977) and Bergström (2014) whereby people investing in the profession make long-term commitments due to cost, knowledge and length of training. Consequently, during the length of time taken to train as an architect, the context has been overtaken by change, yet the ideas surrounding the role are by that time entrenched, making adaptation more difficult. This lag in time can, therefore, in part be considered a contributory factor in the gap between the image and the actuality of practice. During the course of the qualitative research, most of the participants expressed concern over the effect of context on their role. A focus on developing an architect's delineated skills as outlined in section 5.1 as opposed to reinforcing the image were discussed as ways to future-proof the role; creative thought, emphasis on design as a process, and judgement were recurrent themes. The notion that focusing on how these delineated skills, as opposed to the prevailing image, might offer solutions for negotiating challenges to the role are discussed in greater detail in the next section.

\subsection{NEGOTIATION}

\section{Overview of the Study}

Chapter 3 identified and analysed texts which discuss approaches to negotiating the contemporary challenges to architectural practice, the qualitative research built on this information, gathering the experiences from architects working in practice. The theme of negotiation within this thesis, explored four concepts first, challenging the image is considered a means of negotiating the gap between the image and actuality of practice. Re-defining the role in many ways changes its focus. Second, adopting strategic thinking to anticipate and respond to the challenges from the evolving context. Third diversifying or specialising to adjust the focus of a practice in response to fragmentation and competition from emerging alternative service providers. This can be considered to facilitate a re-alignment of expertise within an increasingly complex working context. Finally, the fourth concept discussed as a means of negotiation is research and critical practice; seeking ways to underline and validate design practice, evidencing the value of 
design. An additional consequence of this concept is it opens up opportunities for exploring new directions for the architect's role and new streams of work.

\section{Discussion on Negotiation}

The theoretical research in chapter 3 and the findings in chapter 4 reveal that challenging the image, strategic thinking, diversifying and specialising or research and critical practice, can be viewed as acts to adjust focus within the architect's role and emphasising the core identity of the polymath. They require an outward-looking, adaptive innovator; the focus is therefore on the delineated qualities of creative thinking and judgement. A recurrent position which becomes evident in the qualitative research, is that architects satisfy a distinct function; their range of skills have overlapping interfaces with other disciplines which enable architects to negotiate across different interests and challenges, to fulfil a programme. With this in mind, the undermining of the architect's status signifies that the value of the architect's role is not effectively communicated (Samuel, 2018). The discussions in chapter 4 demonstrate the extent the image of the architect obscures the significance of design as a process and the complexity of skills required to fulfil the role. Negotiation, then, can be seen as a method of challenging the image and communicating the opportunities inherent within the role. This communication should occur both outwardly to reclaim the perceived value in the architect's role, but also inwardly to nurture the inherently transformative identity within the discipline.

Negotiating the image specifically explored how challenging ideas of autonomy and the elite which are identified through this research as particularly problematic and should also form a central part of this communication. To elaborate, autonomy is a prevailing concept through the image since the enlightenment period, however, as well as disregarding the vital relationship with the architect's client, it misrepresents the significance of the architect's skills in negotiating between differing interests as part of the design process. The concept of an elite is equally problematic in an age when the assumed authority of experts is under question (Carrio, 2015; Nichols, 2017; Clarke \& Newman, 2017; Larson, 2018). The profession, therefore, encapsulates a particular view of the profession, based on ethical integrity but also as an elite. Aspects of this have a value, whereby the quality of a service is pre-ordained by an architect's professional status. However, of the architects interviewed, the status ascribed to being a professional was not the reason they entered the profession. The nature of an elite is closed but in order 
to adapt, the architect needs to be outward looking and transformative, ascribing to a particular set professional identity could be considered to restrict this.

\subsection{CONCLUSION}

Since Larson's (1977) analysis of the rise of the professions, research into architecture as a profession grew most notably as a response to the protected functions of the architect coming under scrutiny. The existing work can be categorised either by studies into the culture of architecture (the image) or the culture of architectural practice (the actuality). Of the former Saint (1983), Stevens (1998) and Till (2009) explore the implications of reinforcing idealistic notions of the architectural profession from within. Studies into architectural practice explore the activities of practice, how this differs from the external perceptions (Blau, 1984; Cuff, 1991), but also the effect of changing context on the way practices operate (Gutman, 1988; Symes et al., 1995). More specifically, Sang et al. (2009) and Ahuja et al., (2017) examine the effects of tension between the image and actuality of practice, whereas Hyde (2012) observes ways that architects are adapting their practice as a response to these changes. The field that this thesis covers is, therefore, a topic which is considered significant in understanding the future role of the profession; accordingly, there is an abundance of research exploring the related issues. Within the outlined framework of research, this thesis is located between a cultural examination of the image of the architect and practice-focused exploration into the architect's role. The research builds on an existing understanding of how the image of the architect has developed and contrasts this with the findings of a qualitative study into architects' direct experiences of the actuality of practice. Specifically, through a study of the effects of changing context, the thesis adds to the existing research on how the role might continue to change to ensure its future.

Through this study three things become apparent; first that architects working within the contemporary context recognise that their role is being challenged. Second that the image reveals the adaptive nature of the architect but is reductive and obscures aspects of the role. This leads to misapprehension which suggests that the social value of the architect's role is not being effectively communicated. Third, architects' responses to the challenges are diverse which signifies that negotiation is occurring on an individual basis rather than as a co-ordinated industry-based approach and therefore implies that there is a gap at an institutional level in addressing the challenges to the role. Scrutinising this tension and negotiation reveals which of the architect's innate skills have an intrinsic 
value. The polymathic identity is consistent throughout the development of the image of the architect and the delineated qualities of creative thinking and judgement are valuable in facilitating the architect's transformative identity. This final point addresses the core aim of this thesis and is key to resolving how the image of the architect can adapt synchronously with the evolving context.

\section{Further Research Strands}

This thesis has undertaken an exploration of the core skills that architects have and how they may be harnessed to enable the role to evolve. The study does, however, leave a number of related questions unanswered that are worthy of further research. First, deeper exploration around the specific topic using a broader sampling method and adopting a mixed methods approach would enrich the findings and offer a comparative basis to test the theory and learn more about the issues. This thesis has used a purposive sample (Groat \& Wang, 2002; Patton, 2015) to uncover the experiences of a particular group within the architectural field. A wider study gathering information from architects working within different sectors and also researching conceptions of the image from people outside of architecture such as clients, co-consultants and the general public, would further test the impact of the image of the architect and the extent to which it obscures the social value of the architect's role.

Second, this study is concerned with the experiences of architects within the Anglosphere. The impact of globalisation has been touched on lightly within this study, however an exploration of the impact of this phenomenon as a significant element in the changing context could occur by extending the cross-national nature of this study into other contexts such as Europe and Asia. The purpose of comparing the experiences across different nationalities would be to interrogate whether the challenges experienced by architects within the Anglosphere is repeated across different cultural settings and what the findings reveal about the issues.

On a related topic, this study focusses on how the architect's skills facilitate adaption in the immediate context. The third strand of research would, therefore, expand the subject around a deeper, predictive analysis into the possible role of the architect in the context of the Fourth Industrial Revolution. Further, it would explore the extent to which the use of algorithms for design or intelligent systems in construction might further challenge the architect's role and how architects might adapt to meet these challenges. 


\section{Concluding Remarks}

The subject of this thesis is fertile ground and the possible lines of inquiry which grow from this research can proliferate indefinitely. Therefore the three areas listed above are the most closely connected to the study. The thesis has demonstrated that most architects in practice work beyond the reductive image of an architect; they work subtly using compromise and problem-solving in a delicate and thoughtful way. The multifaceted skills of negotiation, creative thought and judgement are aspects of the role which are not fully communicated through the prevailing image; thus, the social value of the architect's role remains obscure. Similarly, design is considered in the contemporary context to be focused on a product or aesthetic whereas the research reveals how architects who are working in practice regard design to be a process and that the process is complex and valuable. Focusing on the image rather than the process has the unfortunate effect of architectural design being regarded as an applied luxury rather than fundamental to the success of a project.

The architect's role is changing, however as this thesis has discussed, there is much opportunity for reimagining and growth because architects' range of skills enables them to innovate and creatively explore ways of adapting to a perpetually changing context. 


\section{v References}

Ahuja, S, Nikolova, N \& Clegg, S. (2017). Paradoxical identity: The changing nature of architectural work and its relation to architects' identity. Journal of Professions and Organization, 4, 219. doi: 10.1093/jpo/jow013

Agrest, D. (2000). Representation as articulation between theory and practice. In S. Allen \& D. Agrest (Eds.), Practice: architecture. Technique and representation, (pp. 163177). Amsterdam: G\&B Arts International.

Allen, S. (2009). Practice: architecture. Technique and representation (2 ${ }^{\text {nd }}$ ed.). Abingdon: Routledge.

Anthony KH (2001) Designing for diversity: gender, race, and ethnicity in the architectural profession. Urbana: University of Illinois Press.

Awan, N., Schneider, T., \& Till, J. (2013). Spatial agency: other ways of doing architecture.

Abingdon: Routledge.

Bachman, C., \& Bachman, L. (2009). Self-identity, rationalisation and cognitive dissonance in undergraduate architectural design learning. ARQ: Architectural Research Quarterly, 13(3-4), 315-321. doi:10.1017/S1359135510000163

Bauman, Z. (1990). Modernity and ambivalence. Theory, Culture \& Society, 7(2-3), 143-169. doi:10.1177/026327690007002010

Beck, J. (1989). Leon Battista Alberti and the "night sky" at San Lorenzo. Artibus et Historiae, 9-35. doi:10.2307/1483282

Bergström, A. (2014). Architecture and the rise of practice in education. Architectural Theory Review, 19(1), 10-21. doi:10.1080/13264826.2014.894604

Bernstein, P. (2015). Three strategies for new value prepositions of design practice. In P. Deamer (Ed.) The architect as worker: immaterial labor, the creative class, and the politics of design, (pp.209-218). London: Bloomsbury Academic.

Blau, J. R. (1984). Architects and firms: a sociological perspective on architectural practice. Cambridge, Massachusetts: The MIT Press.

Bogdan, R., \& Biklen, S. K. (2007). Qualitative research for education: an introduction to theories and methods. ( $5^{\text {th }}$ ed.). Boston, Massachusetts: Pearson A\&B. 
Boyd, D., \& Danks, S. (2000, September). An analysis of 'the architectural practice' in the construction industry. In Proceedings 16th Annual ARCOM Conference (pp. 6-8).

Bristow, W. (2017). Enlightenment. In E.N. Zalta (Ed.), The Stanford encyclopedia of philosophy. Retrieved from https://plato.stanford.edu/archives/fall2017/entries/enlightenment/

Burr, K. L., \& Jones, C. B. (2010). The role of the architect: changes of the past, practices of the present, and indications of the future. International Journal of Construction Education and Research, 6(2), 122-138. doi:10.1080/15578771.2010.482878

Bush, T. (2012). Authenticity in research-reliability, validity and triangulation. In A. Briggs, M. Morrison \& M. Coleman (Eds.) Research methods in educational leadership and management. ( ${ }^{\text {rd }}$ ed.), (pp. 75-89). London: Sage Publications, inc.

Calinescu, M. (1987). Five faces of modernity: modernism, avant-garde, decadence, kitsch, postmodernism. Durham: Duke University Press.

Carrio, A. (2015). The hunger games: architects in danger. In P. Deamer (Ed.) The architect as worker: immaterial labor, the creative class, and the politics of design, (pp.171-180). London: Bloomsbury Academic.

Caven, V., \& Diop, M. (2012). Architecture: a 'rewarding' career? An Anglo-French comparative study of intrinsic rewards in the architecture profession. Construction Management and Economics, 30(7), 513-523. doi:10.1080/01446193.2011.627356

Caven, V., Navarro-Astor, E., \& Diop, M. (2012). A cross-national study of accommodating and "usurpatory" practices by women architects in the UK, Spain and France. Architectural Theory Review, 17(2-3), 365-377 doi:10.1080/13264826.2012.732588

Chartier, R. (1991). The cultural origins of the French Revolution. Durham: Duke University Press.

Clarke, J., \& Newman, J. (2017). 'People in this country have had enough of experts': Brexit and the paradoxes of populism. Critical Policy Studies, 11(1), 101-116. doi:10.1080/19460171.2017.1282376

Cohen, L., Wilkinson, A., Arnold, J., \& Finn, R. (2005). 'Remember I'm the bloody architect!' Architects, organizations and discourses of profession. Work, Employment and Society, 19(4), 775-796. doi:10.1177/0950017005058065

Coleman, M. (2012). Interviews. In A. Briggs, M. Morrison \& M. Coleman, M. (Eds.) Research methods in educational leadership and management. (3 ${ }^{\text {rd }}$ ed.), (pp. 250265). London: Sage Publications, inc. 
Collier, J. (2006). The art of moral imagination: ethics in the practice of architecture. Journal of Business Ethics, 66(2-3), 307-317. doi:10.1007/s10551-005$5600-4$

Crawford, M. (1991) Can architects be socially responsible? In D. Ghirardo (Ed.) Out of site: A social criticism of Architecture, (pp.27-45). Seattle: Bay Press.

Crinson, M., \& Lubbock, J. (1994). Architecture: art or profession? Three bundred years of architectural education in Britain. Manchester: Manchester University Press.

Crysler, C. G. (1995). Critical pedagogy and architectural education. Journal of Architectural Education, 48(4), 208-217. doi:10.2307/1425383

Cuff, D. (1991). Architecture: the story of practice. Cambridge, Massachusetts: The MIT Press. (1992, 01 July). Divisive tactics: design-production practices in architecture.

Journal of Architectural Education, 45(4), 204-212.

doi:10.1080/10464883.1992.10734516

. (2012). Introduction: Architecture's Double-Bind. In G. Crysler, S. Cairns, \& H. Heynen (Eds.), The Sage handbook of architectural theory (pp. 385-393). London: Sage

Damisch, H., \& Williams, E. (2002). Ledoux with Kant. Perspecta, 33, 10-15. doi: $10.2307 / 1567292$

Davies, J. C. (1962). Toward a theory of revolution. American Sociological Review, 5-19. doi: $10.2307 / 2089714$

De Vries, J. (1994). The industrial revolution and the industrious revolution. The Journal of Economic History, 54(2), 249-270. doi:10.1017/S0022050700014467

Deamer, P. (2012). Detail deliberations. In P. Bernstein, \& P. Dreamer, (Eds.). Building (in) the future: recasting labor in architecture, (pp. 80-89). New York: Princeton Architectural Press.

- (2013). Introduction. In P. Deamer (Ed.) Architecture and capitalism: 1845 to the present, (pp. 24 -49). New York: Routledge.

—. (2015). Work. In P. Deamer (Ed.) The architect as worker: immaterial labor, the creative class, and the politics of design, (pp.61-81). London: Bloomsbury Academic.

Deakin, H., \& Wakefield, K. (2014). Skype interviewing: reflections of two PhD researchers. Qualitative Research, 14(5), 603-616. doi.org:10.1177/1468794113488126 
De Graft-Johnson, A., Manley, S., \& Greed, C. (2005). Diversity or the lack of it in the architectural profession. Construction Management and Economics, 23(10), 1035-1043. doi:10.1080/01446190500394233

Dodd, M. (2015). Double agency: the art school, critical practice and architecture. In H. Harriss \& D. Froud (Eds.). Radical pedagogies: architectural education \& the British tradition, (pp. 21-32). Newcastle-upon-Tyne: RIBA Publications.

Doucet, I., \& Cupers, K. (2009). Agency in architecture: reframing criticality in theory and practice. Footprint, 1-6. doi: 10.7480/footprint.3.1.694

Duffy, F., \& Hutton, L. (1998). Architectural knowledge: the idea of a profession. London: Routledge.

Eisenman, P. (1984). The end of the classical: the end of the beginning, the end of the end. Perspecta, 21, 155-173. doi: 10.2307/1567087

Ellin, N. (1999). Postmodern urbanism. New York: Princeton Architectural Press.

Epstein, S. R. (1998). Craft guilds, apprenticeship, and technological change in preindustrial Europe. The Journal of Economic History, 58(3), 684-713. Retrieved from http://www.jstor.org/stable/2566620

Eraut, M. (2001). Developing professional knowledge and competence. London: Routledge Falmer.

Etikan, I., Musa, S. A., \& Alkassim, R. S. (2016). Comparison of convenience sampling and purposive sampling. American Journal of Theoretical and Applied Statistics, 5(1), 1-4. doi:10.11648/j.ajtas.20160501.11

Frampton, K. (1991). Reflections on the autonomy of architecture: a critique of contemporary production. In D. Ghirardo (Ed.) Out of site: a social criticism of architecture, (pp. 17-26). Seattle: Bay Press.

. (1992). Modern architecture a critical history. (3rd Ed.). London: Thames \& Hudson Ltd.

—. (1993). 20ロ Toward a critical regionalism: six points for an architecture of resistance. In T. Docherty (Ed.), Postmodernism: a reader, (pp. 268-280).

Abingdon: Routledge. . (2012). Intention, craft and rationality. In P. Bernstein, \& P. Dreamer, (Eds.). Building (in) the future: recasting labor in architecture, (pp. 28-37). New York: Princeton Architectural Press.

Franck, K. A., \& Lepori, R. B. (2007). Architecture from the inside out $\left(2^{\text {nd }}\right.$ ed.). Chichester, England: Wiley. 
Frascari, M., Hale, J., \& Starkey, B. (Eds.). (2013). From models to drawings: imagination and representation in architecture. London: Routledge.

Froud, D. (2015). 'You will see pictures of what is bad and what is good': architects, 'normal people' and the politics of urban space. In H. Harriss \& D. Froud (Eds.). Radical pedagogies: architectural education \& the British tradition, (pp. 45-56). Newcastle upon Tyne: RIBA Publications.

Gadamer, H. G., (2013). (2nd ed.) Truth and method. London: Bloomsbury Academic. (Original publication 1975).

Gelderblom, O., \& Grafe, R. (2010). The rise and fall of the merchant guilds: re-thinking the comparative study of commercial institutions in premodern Europe. Journal of Interdisciplinary History, 40(4), 477-511. doi:10.1162/jinh.2010.40.4.477

Ghirardo, D. (1996). Architecture after modernism. London: Thames and Hudson.

Gloster, D. (2015). Preface: instability, innovation and emergence within architectural eductation. In H. Harriss \& D. Froud (Eds.). Radical pedagogies: architectural education \& the British tradition, (pp. 6-9). Newcastle upon Tyne: RIBA Publications.

Goldschmidt, G. (2003, November). Expert knowledge or creative spark? Predicaments in design education. In Expertise in Design, Design Thinking Research Symposium (Vol. 6, pp. 17-19). Retrieved from http://home.fa.utl.pt/ franc/de1/ext01/ggcriativo.pdf

Goudeau, J. J. W. (2015). The matrix regained: reflections on the use of the grid in the architectural theories of Nicolaus Goldmann and Jean-Nicolas-Louis Durand. Architectural Histories, 3, 1-17. doi: 10.5334/ah.cl

Gough, T. (2013). Architecture as a strong discipline. Architecture and Culture, 1(1), 20-41. doi:10.2752/175145213X13760412749953

Grafton, A. (2002). Leon Battista Alberti: master builder of the Italian Renaissance. Cambridge, Massachusetts: Harvard University Press.

Groat, L. N. (1993). Architecture's resistance to diversity: a matter of theory as much as practice. Journal of architectural education, 47(1), 3-10. doi: 10.2307/1425223

Groat, L. \& Wang, D. (2002) Architectural research methods. New York: John Wiley \& Sons, Inc. 
Gutman, R. (1988) Architectural practice a critical view. New York: Princeton Architectural Press.

Habermas, J. (2005). Modernity—an incomplete project. In S. P. Hier (Ed.) Contemporary sociological thought: themes and theories, (pp.165-174). Toronto: Canadian Scholars' Press Inc. (Originally published 1983).

Hantrais, L. (1999) Contextualisation in cross-national comparative research. International Journal of Social Research Methodology, 2(2), 93-108. doi:10.1080/136455799295078 Harries, K. (2002). Sphere and cross: Vitruvian reflections on the Pantheon type. In G. Dodds, R. Tavernor \& J. Rykwert (Eds.). Body and Building: Essays on the Changing Relation of Body and Architecture, (pp. 149-162). Cambridge, Massachussets: MIT Press.

Harriss, H., \& Froud, D. (2015). Radical pedagogies: architectural education \& the British tradition. Newcastle upon Tyne: RIBA Publications.

Harvey, D. (1990). The condition of postmodernity: an enquiry into the conditions of cultural change. Cambridge, Massachusetts: Blackwell.

Hay, R., Samuel, F., Watson, K. J., \& Bradbury, S. (2018). Post-occupancy evaluation in architecture: experiences and perspectives from UK practice. Building Research \& Information, 46(6), 698-710. doi: 10.1080/09613218.2017.1314692

Heynen, H. (1999) Architecture and modernity. Cambridge, Massachusetts: MIT.

Hickson, C. R., \& Thompson, E. A. (1991). A new theory of guilds and European economic development. Explorations in Economic History, 28(2), 127-168. doi:10.1016/0014-4983(91)90015-B

Hill, J. (2003). Actions of architecture: architects and creative users. New York: Routledge.

Hill, D. (2012). Foreword. In R. Hyde, Future practice: conversations from the edge of architecture, (pp.7-15). New York: Routledge.

Hirst, P. (2005). Space and power: politics, war and architecture. Malden, Massachusetts: Polity Press.

Hughes, A. (1986). 'An academy for doing'. I: the Accademia del Disegno, the guilds and the Principate in sixteenth-century Florence. Oxford Art Journal, 9(1), 3-10.

Huxtable, A. L. (2008). On architecture: collected reflections on a century of change. New York: Walker Publishing Co. inc.

Hyde, R. (2013). Future practice: conversations from the edge of architecture. New York: Routledge. 
Imrie, R. (2007). The interrelationships between building regulations and architects' practices. Environment and Planning B: Planning and Design, 34(5), 925-943. doi.org:10.1068/b33024

Imrie, R., \& Street, E. (2014). Autonomy and the socialisation of architects. The Journal of Architecture, 19(5), 723-739. doi:10.1080/13602365.2014.967271

Jamieson, C. (2011). RIBA: The future for architects. London:RIBA

Jencks, C. (1973). Modern movements in architecture. Middlesex: Penguin Ltd. (2006). The iconic building is here to stay. City, 10(1), 3-20. doi:10.1080/13604810600594605

Jenkins, P., \& Forsyth, L. (2009). Architecture, participation and society. London: Routledge. Jenks, C. J. (2013). Working with transcripts: An abridged review of issues in transcription. Language and Linguistics Compass, 7(4), 251-261. doi:10.1111/lnc3.12023

Johnson, B., \& Turner, L. A. (2003). Data collection strategies in mixed methods research. In T. Abbas \& T. Charles (Eds.) Handbook of mixed methods in social and behavioural research, (pp. 297-319). Thousand Oaks, California: Sage Publications, inc.

Johnson, P. A. (1994). The theory of architecture: concepts themes \& practices. New York: Van Nostrand Reinhold.

Jones, P. (2009). Putting architecture in its social place: a cultural political economy of architecture. Urban Studies, 46(12), 2519-2536. doi:10.1177/0042098009344230

Kapp, S., Baltazar, A. P., \& Morado, D. (2008). Architecture as critical exercise: little pointers towards alternative practices. Field, 2(1), 7-29. Retrieved from http:/ / field-journal.org/wp-content/uploads/2016/07/Architecture-as-CriticalExercise_MOM.pdf

King, N. (2004). Using templates in the thematic analysis of text. In C. Cassell \& G. Symon (Eds.) Essential guide to qualitative methods in organizational research, (pp. 25670). London: Sage.

Kostof, S. (1977). The architect: chapters in the history of the profession. New York: Oxford University Press.

Kvale, S. (2007) Doing interviews. London: Sage Publications, inc.

Lorne, C. (2017). Spatial agency and practising architecture beyond buildings. Social \& Cultural Geography, 18(2), 268-287. doi:10.1080/14649365.2016.1174282 
Larson, M. S. (1977). The Rise of Professionalism: A Sociological Analysis. (Vol. 233). Berkeley, California: UC Press.

- (2018). Professions today: self-criticism and reflections for the future. Sociologia, Problemas e Práticas, (88), 27-42. doi:10.7458/SPP20188814796

Le Corbusier \& Etchells, F. (1986). Towards a new architecture. New York: Dover Publications.

Lupton, B. (2000). Maintaining masculinity: men who do 'women's work'. British Journal of Management, 11, 33-48. doi:10.1111/1467-8551.11.s1.4

Lyotard, J. F. (1985). The sublime and the avant-garde. Paragraph, 6(1), 1-18. Retrieved from https://www.jstor.org/stable/43151610

Madrazo, L. (1994). Durand and the science of architecture. Journal of Architectural Education, 48(1), 12-24. doi: 10.2307/1425306

McEwen, I. K. (2003). Vitruvius: writing the body of architecture. Cambridge, Massachusetts: MIT press.

McLeod, M. (1989). Architecture and politics in the Reagan era: from postmodernism to deconstructivism. Assemblage, (8), 23-59. doi:10.2307/3171013

McLeod, M. (1983). “Architecture or Revolution”: Taylorism, technocracy, and social change. Art journal, 43(2), 132-147. doi:10.2307/776649

Miller, R. (1988). Burnham, Sullivan, Roark, and the myth of the heroic architect. Art Institute of Chicago Museum Studies, 13(2), 87-95. doi:10.2307/4115893

Murphy, D. (2016, June 4). The end of utopia. The Architectural Review. Retrieved from https://www.architectural-review.com/essays/viewpoints/the-end-ofutopia/10006220.article

Morse, J. M., Barrett, M., Mayan, M., Olson, K., \& Spiers, J. (2002). Verification strategies for establishing reliability and validity in qualitative research. International Journal of Qualitative Methods, 1(2), 13-22. https://doi.org/10.1177/160940690200100202

Nesbitt, K. (1995). The sublime and modern architecture: unmasking (an aesthetic of) abstraction. New Literary History, 26(1), 95-110. Retrieved from www.jstor.org/stable/20057270

Nichols, T. (2017). How America lost faith in expertise: and why that's a giant problem. Foreign Aff., 96, 60. Retrieved from https://heinonline.org/HOL/LandingPage?handle=hein.journals/fora96\&div= 42\&id $=$ \&page $=$ 
Nicol, D, \& Pilling, S. (2000). Architectural education and the profession: preparing for the future. In D. Nicol \& S. Pilling (Eds.) Changing architectural education: Towards a new professionalism, (pp.1-26). Bloomsbury Publishing. London: Spon Press.

Onwuegbuzie, A. J., \& Collins, K. M. (2007, 02 June). A typology of mixed methods sampling designs in social science research. The Qualitative Report, 12(2), 281-316. Retrieved from http://nsuworks.nova.edu/tqr/vol12/iss2/9

Padgett, J. F., \& Ansell, C. K. (1993). Robust action and the rise of the Medici, 14001434. American Journal of Sociology, 98(6), 1259-1319. doi: 10.1086/230190

Pallasmaa, J. (2011). The embodied image. Chichester, England: John Wiley \& Sons.

Patterson, R. (1997). What Vitruvius said. The Journal of Architecture, 2(4), 355-373. https://doi.org/10.1080/136023697374360

Patton, M.Q. (2015). Qualitative research and evaluation methods: integrating theory and practice $4^{\text {th }}$ ed.). Thousand Oaks, California: Sage Publications, inc.

Perez-Gomez, A. (1982). Architecture as drawing. Journal of Architectural Education, 36(2), 2-7. doi:10.2307/1424613

- (2005). Questions of representation: the poetic origin of architecture. arq: Architectural Research Quarterly, 9(3-4), 217-225. doi:10.1017/S1359135505000278

Poland, B. D. (2003). Transcription quality. In J. Holstein \& J. F. Gubrium (Eds.) Inside interviewing: New lenses, new concerns, 267-287. Sage Publications, inc.

Ponzini, D. (2014). The values of starchitecture: Commodification of architectural design in contemporary cities. Organizational Aesthetics, 3(1), 10-18. Retrieved from https://digitalcommons.wpi.edu/oa/vol3/iss1/4

Powell, H., \& Prasad, S. (2010). “As seen on TV.” The celebrity expert: how taste is shaped by lifestyle media. Cultural politics, 6(1), 111-124. doi:10.2752/175174310X12549254318908

Powers, A. (2015). The fiction of architectural education. In H. Harriss \& D. Froud (Eds.). Radical pedagogies: architectural education \& the British tradition, (pp. 3-20). Newcastle upon Tyne: RIBA Publications.

Price, C. (2017). Has the architectural profession a future? In S. Hardingham (2017) Cedric Price works 1952-2003: A forward-minded retrospective, (pp.238-240). London: AA Publications. (Original lecture presented 1975)

Rawes, P. (2007). Reflective subjects in Kant and architectural design education. Journal of Aesthetic Education, 74-89. Retrieved from www.jstor.org/stable/4140241 
Reinmuth, G. (2017). Relationality and architecture: how refocusing the discipline might reverse the profession's seemingly unstoppable trajectory of decline. Architectural Theory Review, 21(1), 89-107. https://doi.org/10.1080/13264826.2017.1288147

RIBA (2017). Practice Resilience: how architects survive and thrive during challenging times.

Retrieved from https://www.architecture.com/knowledge-and-

resources/resources-landing-page/practice-resilience

(2018). Future trends survey: October 2018. Retrieved from

https://www.architecture.com/knowledge-and-resources/resources-landing-

page/future-trends-survey-2018

Richards, L. (1999). Data alive! The thinking behind NVivo. Qualitative Health

Research, 9(3), 412-428. https://doi-

org.helicon.vuw.ac.nz/10.1177/104973239900900310

Richards, W. (2015, September 18). Superhuman? We need a new definition of the architectural hero. American Institute of Architects: Architect Magazine. Retrieved from https://www.architectmagazine.com/aia-

architect/aiafuture/superhuman_o

Rudofsky, B. (1964). Architecture without architects: a short introduction to non-pedigreed architecture.

Albuquerque: UNM Press.

Saint, A. (1983). The image of the Architect. Yale University Press. Retrieved from https://www.jstor.org/stable/j.ctt1ww3w0s.12

Salingaros, N. A., \& Mehaffy, M. W. (2006). Geometrical fundamentalism. In N. Salingaros. A theory of architecture. Solingen:UMBAU-VERLAG Harald Püschel.

Salingaros, N. A., \& Mikiten, T. M. (2006). Darwinian processes and memes in Architecture: A mimetic theory of modernism. In N. Salingaros. A theory of Architecture. Solingen:UMBAU-VERLAG Harald Püschel.

Salomon, D. (2012). Plural profession, discrepant practices. In G. Crysler, S. Cairns, \& H. Heynen (Eds.), The Sage handbook of architectural theory (pp. 430-443.). London: Sage.

Samuel, F. (2018). Why architects matter: Evidencing and communicating the value of architects. Oxford: Routledge.

Sandelowski, M. (1995). Sample size in qualitative research. Research in Nursing \& Health, 18(2), 179-183. https://doi.org/10.1111/puar.12955 
Sang, K., Ison, S., Dainty, A., \& Powell, A. (2009). Anticipatory socialisation amongst architects: a qualitative examination. Education + Training, 51(4), 309-321. https://doi.org/10.1108/00400910910964584

Saunders, W. S. (1997). Rem Koolhaas's writing on cities: poetic perception and gnomic fantasy. Journal of Architectural Education, 51(1), 61-71. doi:10.1080/10464883.1997.10734747

Schneider, T., \& Till, J. (2009). Beyond discourse: notes on spatial agency. Footprint, $97-$ 112. doi:10.7480/ footprint.3.1.702

Schön, D. A. (1995). The reflective practitioner: how professionals think in action. Aldershot, Hants.: Ashgate.

Schumacher, P (1997). Materialism versus morality, part 2. Lecture delivered at Architectural Association, London as part of the graduate school etbics lecture series. Retrieved from https://www.patrikschumacher.com/Texts/morality2.htm

Seale, C. (1999). Quality in qualitative research. Qualitative inquiry, 5(4), 465-478. doi:10.1177/107780049900500402

Smith, M. E. (2014). Peasant mobility, local migration and premodern urbanization. World Archaeology, 46(4), 516-533. doi:10.1080/00438243.2014.931818

Smitheram, J., Kidd, A. N., \& Lam, S. (2018). Celebrified homes: architecture and spacing celebrities. Celebrity Studies, 9(3), 375-390. doi: 10.1080/19392397.2018.1439393

Smitheram, J., \& Nakai Kidd, A. (2019). On time within an architectural community. Time \& Society, 0(0), 1-23. doi:10.1177/0961463X18820737

Stevens, G. (2002). The favoured circle: The social foundations of architectural distinction. Cambridge, Massachusetts: MIT press.

Stead, N., \& Richards, M. (2014). Valuing architecture: taste, aesthetics and the cultural mediation of architecture through television. Critical Studies in Television, 9(3), 100112. doi:10.7227/CST.9.3.10

Sturges, J. (2013). A matter of time: young professionals' experiences of long work hours. Work, Employment and Society, 27(2), 343-359. https://doi.org/10.1177/0950017012460318

Styhre, A., \& Gluch, P. (2009). Creativity and its discontents: professional ideology and creativity in architect work. Creativity and Innovation Management, 18(3), 224-233. Retrieved from http://publications.lib.chalmers.se/records/fulltext/96816/local_96816.pdf 
Sudjic, D. (2005). The edifice complex: how the rich and powerful-and their architects-shape the world. London: Allen Lane.

Symes, M., Eley, J., \& Seidel, A. (1995). Architects and their practices: a changing profession. Oxford: Butterworth Architecture.

Till, J. (2009). Architecture depends (Vol. 55). Cambridge, Massachusetts: MIT press.

Tombesi, P. (2001). A true south for design? The new international division of labour in architecture. ARQ: Architectural Research Quarterly, 5(2), 171-180. doi:10.1017/S135913550100119

- (2006). Good thinking and poor value: on the socialization of knowledge in construction. Building Research \& Information, 34(3), 272-286. https://doi.org/10.1177/107780049900500402

- (2012). On the cultural separation of design labor. In P. Deamer, \& P.G. Bernstein (Eds.) Building (in) the future: recasting labor in architecture, (pp. 117-136). New York: Princeton Architectural Press.

Vesely, D. (2002) The architectonics of embodiment. In G. Dodds, R. Tavernor \& J. Rykwert (Eds.). Body and building: essays on the changing relation of body and architecture, (pp. 28-43). Cambridge, Massachussets: MIT Press.

- (2004). Architecture in the age of divided representation: the question of creativity in the shadow of production. Cambridge, Massachussets: MIT press.

Vidler, A. (2002). The Ledoux effect: Emil Kaufmann and the claims of Kantian autonomy. Perspecta, 33, 16-29. doi:10.2307/1567293

- (2008). Histories of the immediate present: inventing architectural modernism, 1930-1975. Cambridge, Massachussets: MIT Press.

Vitruvius P., Rowland, I. D., \& Howe, T. N. (1999). Vitruvius: 'Ten Books on Architecture'. New York: Cambridge University Press.

Wallerstein, I. (1974). The rise and future demise of the world capitalist system: concepts for comparative analysis. Comparative Studies in Society and History, 16(4), 387-415. https://doi.org/10.1017/S0010417500007520

Watts, J. H. (2009). 'Allowed into a man's world' meanings of work-life balance: perspectives of women civil engineers as 'minority' workers in construction. Gender, Work \& Organization, 16(1), 37-57. https://doi.org/10.1111/j.1468-0432.2007.00352.x 
Wilson, C. (1989). The Newtonian achievement in astronomy. In R. Taton, C. Wilson, M. Hoskin (Eds.), Planetary astronomy from the Renaissance to the rise of astrophysics. Part A: Tycho Brabe to Newton (pp. 233-274). Cambridge: Cambridge University Press.

Winch, G., \& Schneider, E. (1993). Managing the knowledge-based organization: the case of architectural practice. Journal of Management Studies, 30(6), 923-937. doi:10.1111/j.1467-6486.1993.tb00472.x

Wiscome, T. (2006, 01 January). Emergent Models of Architectural Practice. Perspecta, 38, 57-68. doi:10.2307/40482417 
vi Appendix-Architects Selection Matrix

\begin{tabular}{|c|c|c|c|c|c|c|c|}
\hline interviewee reference & size of practice* & practice focus & Age of practice & studio style & Teaching / Research & country & participant \\
\hline Architect\#1NZM & band $1-2$ & domestic & $10+$ & Director led & $\begin{array}{c}\text { Practice research } \\
\text { group }\end{array}$ & $\mathrm{NZ}$ & $\checkmark$ \\
\hline Architect\#2UKM & band $1-2$ & offices \& domestic & $5+$ & Director led & $\begin{array}{l}\text { Health \& Well-being } \\
\text { \& CNC processes }\end{array}$ & UK & $\checkmark$ \\
\hline Architect\#3NZF & band $1-2$ & domestic & $10+$ & Director led & $\begin{array}{l}\text { Practice research } \\
\text { group }\end{array}$ & $\mathrm{NZ}$ & $\checkmark$ \\
\hline Architect\#4NZM & band 6 & housing & $50+$ & studio groups & $\begin{array}{l}\text { Lectures \& practice } \\
\text { group }\end{array}$ & $\mathrm{NZ}$ & $\checkmark$ \\
\hline Architect\#5NZM & band 6 & multiple & $25+$ & $\begin{array}{l}\text { Director led studio } \\
\text { groups }\end{array}$ & Research lab & $\mathrm{NZ}$ & $\checkmark$ \\
\hline Architect\#6UKF & band 3 & multiple & $5+$ & collaborative & $\begin{array}{l}\text { Design guide research } \\
\& \text { university role }\end{array}$ & UK & $\checkmark$ \\
\hline Architect\#7UKM & band 5 & multiple & $15+$ & $\begin{array}{l}\text { Director led } \\
\text { Charettes }\end{array}$ & $\begin{array}{c}\text { Collaborative practice } \\
\text { visiting tutor and } \\
\text { lectures } \\
\end{array}$ & UK & $\checkmark$ \\
\hline Architect\#8UKM & band 5 & multiple & $50+$ & co-operative & $\begin{array}{c}\text { Self-directed research } \\
\text { practice } \\
\text { apprenticeship } \\
\end{array}$ & UK & $\checkmark$ \\
\hline Architect\#0NZF & band 3 & multiple & $15+$ & $\begin{array}{l}\text { Director led } \\
\text { Charettes }\end{array}$ & Prefabricaction & $\mathrm{NZ}$ & $x$ \\
\hline Architect\#0UKF & band 6 & multiple & $50+$ & $\begin{array}{l}\text { Director led studio } \\
\text { groups }\end{array}$ & $\begin{array}{l}\text { CABE, prefabrication } \\
\text { and housing research }\end{array}$ & UK & $x$ \\
\hline
\end{tabular}

* Based on RIBA Chartered Practice Accreditation Criteria

$\begin{array}{cc}\text { Band } & \text { no. Architectura } \\ 1 \& 2 & 1-5 \\ 3 & 6-10 \\ 4 & 11-25 \\ 5 & 26-50 \\ 6 & 51-150+\end{array}$




\section{vii Appendix-Sample Interview Questions}

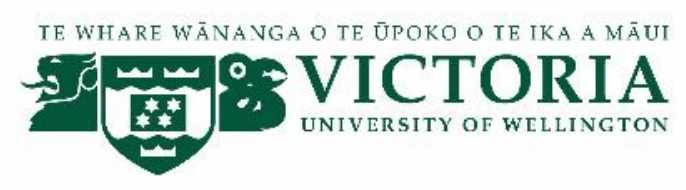

\section{SAMPLE INTERVIEW QUESTIONS}

This is research that asks about the continuing role of the architect in the modern building industry. The directed conversations with architects will entail three elements:

4. Establishing perceived differences between the image and the actuality of architectural practice.

5. Ascertain practitioners' experience of and response to changing conditions.

6. Discuss strategies to counter or adapt to new and emerging conditions.

\section{Example of questions:}

1. What, in your opinion, is the prevailing image of an architect?

2. How did you discover architecture as a profession and why did you decide to embark on a career in architecture?

3. In which ways do you reinforce the image and in which ways do you challenge it?

4. Has your career progressed as you expected?

5. What are the greatest challenges to the work you do?

6. What are the greatest challenges to the practice itself?

7. How do you see the practice adapting to these challenges?

8. How does the practice generally acquire work?

9. What role does the image play in acquiring work?

10. What aspect of the profession do you consider to be most rewarding?

11. What aspect of the profession do you find most frustrating?

12. Looking towards the future, how do you predict your role and / the practice will evolve?

13. How do you foresee a sustainable business model emerges from this? 
